\title{
Choice-selective sequences dominate in cortical relative to thalamic inputs to nucleus accumbens, providing a potential substrate for credit assignment
}

\author{
Nathan F. Parker ${ }^{1 *}$, Avinash Baidya ${ }^{3,4 *}$, Julia Cox ${ }^{1 *}$, Laura Haetzel ${ }^{1}$, Anna Zhukovskaya ${ }^{1}$, \\ Malavika Murugan ${ }^{1}$, Ben Engelhard ${ }^{1}$, Mark S. Goldman ${ }^{3}$, Ilana B. Witten ${ }^{1,2 \#}$
}

\author{
Affiliations: \\ ${ }^{1}$ Princeton Neuroscience Institute \\ ${ }^{2}$ Department of Psychology \\ Princeton University, Princeton NJ 08544 \\ ${ }^{3}$ Center for Neuroscience \\ ${ }^{4}$ Department of Physics and Astronomy \\ University of California Davis, Davis, CA 95616 \\ \# correspondence: iwitten@princeton.edu, msgoldman@ucdavis.edu \\ * these authors contributed equally
}

How are actions linked with subsequent outcomes to guide choices? The nucleus accumbens, which is implicated in this process, receives glutamatergic inputs from the prelimbic cortex and midline regions of the thalamus. However, little is known about what is represented in these input pathways. By comparing these inputs during a reinforcement learning task in mice, we discovered that prelimbic cortical inputs preferentially represent actions and choices, whereas midline thalamic inputs preferentially represent cues. Choice-selective activity in the prelimbic cortical inputs is organized in sequences that persist beyond the outcome. Through computational modeling, we demonstrate that these sequences can support the neural implementation of temporal difference learning, a powerful algorithm to connect actions and outcomes across time. Finally, we test and confirm predictions of our circuit model by direct manipulation of nucleus accumbens input neurons. Thus, we integrate experiment and modeling to suggest a neural solution for credit assignment. 


\section{Introduction}

Multiple lines of experimental evidence indicate that the nucleus accumbens (NAc, part of the ventral striatum) is critical to reward-based learning and decision-making (Apicella et al., 1991; Cador et al., 1989; Carelli et al., 1993; Cox and Witten, 2019; Di Ciano et al., 2001; Everitt et al., 1991; Parkinson et al., 1999; Phillips et al., 1993, 1994; Robbins et al., 1989; Roitman et al., 2005; Setlow et al., 2003; Stuber et al., 2011; Taylor and Robbins, 1986). The NAc is a site of convergence of glutamatergic inputs from a variety of regions, including the prefrontal cortex and the midline thalamus, along with dense dopaminergic inputs from the midbrain (Brog et al., 1993; Do-Monte et al., 2017; Groenewegen et al., 1980; Hunnicutt et al., 2016; Otis et al., 2017; Phillipson and Griffiths, 1985; Poulin et al., 2018; Reed et al., 2018; Swanson, 1982; Wright and Groenewegen, 1995; Zhu et al., 2016).

An important mechanism underlying reward-based learning and decision-making is thought to be dopamine-dependent synaptic plasticity of glutamatergic inputs to the NAc that are co-active with a reward prediction error (RPE) in dopamine neurons (Fisher et al., 2017; Gerfen and Surmeier, 2011; Reynolds and Wickens, 2002; Russo et al., 2010). Such strengthening of glutamatergic inputs is thought to be central to learning, allowing actions that are followed by a rewarding outcome to be more likely to be repeated in the future (Britt et al., 2012; MacAskill et al., 2014; Steinberg et al., 2013; Tsai et al., 2009; Witten et al., 2011).

A central question in reinforcement learning is how actions and outcomes become associated with each other, even when they are separated in time (Asaad et al., 2017; Gersch et al., 2014; Sutton, 1988; Wörgötter and Porr, 2005). A possible mechanism that could contribute to solving this problem of temporal credit assignment in the brain is that neural activity in the glutamatergic inputs to the NAc provide a neural memory trace of previous actions. This could allow action representations from glutamatergic inputs and outcome information from dopaminergic inputs to overlap in time.

Whether glutamatergic inputs to the NAc indeed represent memories of previous actions is unclear. More broadly, what information is carried by glutamatergic inputs to the NAc during reinforcement learning, and whether different inputs provide overlapping or distinct streams of information, has not been examined systematically. To date, there have been relatively few recordings of cellular-resolution activity of glutamatergic inputs to the NAc during reinforcement learning, nor comparison of multiple inputs within the same task, nor examination of the timescale with which information is represented within and across trials. Furthermore, if glutamatergic inputs do indeed provide memories of previous actions, construction of a neurally plausible instantiation of an algorithm for credit assignment based on the measured signals remains to be demonstrated (for review of biological instantiation of reinforcement learning algorithms, see (Joel et al., 2002)).

To address these gaps, we recorded from glutamatergic inputs to the NAc during a probabilistic reversal learning task that we previously demonstrated was dopamine-dependent. In this task, dopamine neurons that project to the NAc encode RPE, and inhibition of dopamine neurons substitutes for a negative RPE (Parker et al., 2016). To compare activity in major cortical and thalamic input to the NAc core, here we combined a retrograde viral targeting strategy with cellular-resolution imaging to examine the input from prelimbic cortex ("PL-NAc", part of medial prefrontal cortex) and that from the midline regions of the thalamus ("mTH-NAc"). We found that PL-NAc neurons preferentially encode actions and choices relative to mTH-NAc neurons, with choice-selective sequential activity that persists until the start of the subsequent trial. The long timescale through which a prior action is encoded in cortical inputs to the NAc provides the information to bridge actions, 
outcomes, and the subsequent choice. In addition, we demonstrated with computational modeling that these choice-selective sequences can contribute to a concrete neural instantiation of temporal difference (TD) learning, a powerful reinforcement learning algorithm that allows appropriate learning of the association of actions and outcomes separated in time. Finally, we test and confirm a prediction of our model through direct optogenetic manipulation of PL-NAc neurons. Thus, by recording and manipulating glutamatergic inputs to the NAc and integrating these data with computational modeling, we provide a specific proposal for how TD learning could be implemented by neural circuitry.

\section{Results}

Cellular resolution imaging of glutamatergic inputs to the NAc during a probabilistic reversal learning task

Mice performed a probabilistic reversal learning task while inputs from thalamus or cortex were imaged (Figure 1a). A trial was initiated when the mouse entered a central nose poke, which prompted the presentation of a lever on either side. Each lever had either a high $(70 \%)$ or low $(10 \%)$ reward probability, with the identity of the high and low probability levers reversing in an unsignaled manner after a variable number of trials (see Methods for block transition probabilities). After a variable delay (0-1s), either a sound (CS+) was presented at the same time as a reward was delivered to a central reward port, or another sound (CS-) was presented that signaled the absence of reward.

As expected, mice reversed the lever they were more likely to press following block transitions (Figure 1b,c). Similarly, mice were significantly more likely to return to the the previously chosen lever (i.e. stay) following rewarded, as opposed to unrewarded, trials (Figure 1d; $p<0.0001$ : paired, two-tailed t-test between stay probabilities following rewarded and unrewarded trials across mice, $\mathrm{n}=16$ mice), meaning that, as expected, mice were using previous choices and outcomes to guide behavior. A logistic regression to predict choice based on previous choices and outcomes indicated that mice relied on $\sim 3$ previous trials to guide their choices (Figure 1e; see Methods for choice regression details).

To image activity of glutamatergic input neurons to the NAc during this behavior, we injected a retroAAV or CAV2 to express Cre-recombinase in the NAc as well as an AAV2/5 to Cre-dependently express GCaMP6f in either the PL or mTH (Figure 1f). A gradient refractive index (GRIN) lens was implanted above either the PL or $\mathrm{mTH}$ (see Supplementary Figure 1 for implant locations), and a head-mounted miniature microscope was used to image activity in these populations during behavior (Figure 1f, $n=278$ neurons in PL-NAc from $n=7$ mice, $n=256$ neurons in $m$ TH-NAc from $n=9$ mice). An example field of view from a single recording session is shown for both PL-NAc neurons as well as mTH-NAc neurons (Figure 1g). Behavior between mice in the PL-NAc versus mTH-NAc cohorts was similar (Supplementary Figure 2).

Actions are preferentially represented by PL-NAc neurons, while reward-predicting stimuli are preferentially represented by $m \mathrm{TH}-\mathrm{NAc}$ neurons

Individual PL-NAc and mTH-NAc neurons displayed elevated activity when time-locked to specific behavioral events in the task (Figure 2a). However, given the correlation between the timing of task events, as well as the temporal proximity of events relative to the time-course of GCaMP6f, we built a linear encoding model to properly relate neural activity to each event (Engelhard et al., 2019; Krumin et al., 2018; Lovett-Barron et al., 2019; Musall et al., 2019; Park et al., 2014; Parker et al., 2016; Pinto and Dan, 2015; Sabatini, 2019; Steinmetz et al., 2019). Briefly, time-lagged versions of each behavioral event (nosepoke, lever press, etc) were used to predict the GCaMP6f fluorescence in each neuron using a linear regression. This allowed us to obtain "response kernels", which related 
each event to the GCaMP6f fluorescence in each neuron, while removing the potentially confounding (linear) contributions of correlated task events (Figure $\mathbf{2 b}$; see Methods for model details).

To visualize the response kernels, we plotted them as a heatmap, where each row was the response kernel for a particular neuron associated with each behavioral event. This heatmap was then ordered by the time of peak kernel value across all behavioral events. Visual observation revealed a clear difference between the PL-NAc and mTH-NAc populations - PL-NAc neurons were robustly modulated by the action-events in our task (Figure 2c; kernel values associated with 'nose poke, 'ipsilateral lever press', 'contralateral lever press' and 'reward consumption') while mTH-NAc neurons appeared to be most strongly modulated by the stimulus-events, specifically the positive reward auditory cue (Figure $\mathbf{2 d}$, kernel values associated with 'CS+').

Importantly, examination of the GCaMP6f fluorescence time-locked to each behavioral event (rather than the encoding model-derived response kernels) revealed similar observations of action encoding in PL-NAc and CS+ encoding in mTH-NAc (Figure 2e,f). While this time-locked GCaMP6f heatmap displays neurons which appear to respond to multiple events (Figure 2e, see neurons approximately 50-100 that show elevated activity to 'ipsilateral lever press', 'levers out' and 'nose poke'), this impression is likely a result of the temporal correlation between neighboring behavioral events, which our encoding model accounts for. To illustrate this, we applied our encoding model on a population of simulated neurons that responded only to the lever press events. In this simulated data, we observed a similar multi-peak heatmap when simply time-locking the simulated GCaMP6f fluorescence, but this multi-peak effect is eliminated by the use of our encoding model, which recovers the true relationship between GCaMP6f fluorescence and behavior in the simulated data (Supplementary Figure 3).

This encoding model was used to identify neurons in the PL-NAc and mTH-NAc populations that were significantly modulated by each event in our task (significance was assessed by comparing the encoding model with and without each task event, see Methods). We found that a similar fraction of PL-NAc and mTH-NAc neurons were modulated by at least one task event (Figure 2g; PL-NAc: $n=121 / 278$ neurons from 7 mice; mTH-NAc: $n=98 / 256$ neurons from 9 mice). Of these neurons that were selective to at least one task event, the selectivity for actions versus sensory stimuli differed between the two populations (Figure $\mathbf{2 g}, \mathbf{h}$ ). In particular, more PL-NAc neurons were modulated by at least one action event (nose poke, ipsilateral lever press, contralateral lever press and reward consumption; 102/121 PL-NAc neurons; 60/98 mTH-NAc neurons; $\mathrm{P}=0.0001$ : two-proportion Z-test comparing fraction of action-modulated neurons between PL-NAc and mTH-Nac). In contrast a significantly larger fraction of $\mathrm{mTH}-\mathrm{NAc}$ neurons were modulated by at least one stimulus cue (levers out, CS+ and CS-; 51/98 mTH-NAc neurons; 40/121 PL-NAc neurons; P=0.005, two-proportion Z-test comparing fraction of stimulus-modulated neurons between PL-NAc and mTH-NAc).

\section{PL-NAc neurons preferentially encode choice relative to $\mathrm{mTH}$-NAc neurons}

This preferential representation of actions in PL-NAc relative to mTH-NAc suggests that lever choice (contralateral versus ipsilateral to the recording site) could also be preferentially encoded in PL-NAc. Indeed, a significantly larger fraction of neurons were choice-selective in PL-NAc compared

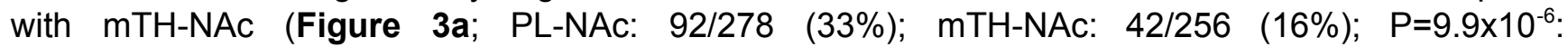
two-proportion Z-test; significant choice-selectivity was determined with a nested comparison of the encoding model with and without choice information, see Methods). A logistic regression population decoder supported this observation of preferential choice-selectivity in PL-NAc relative to mTH-NAc. Choice decoding using neural activity of simultaneously recorded PL-NAc neurons was significantly more accurate compared with decoding using mTH-NAc activity (Figure 3b; PL-NAc: $72 \pm 3 \%$, mTH-NAc: $60 \pm 2 \%$, choice decoding accuracy from a logistic regression with activity from multiple, random selections of 10 simultaneously imaged neurons, mean \pm s.e.m. across mice; $P=0.0065$ : 
unpaired, two-tailed t-test comparing peak decoding accuracy across mice between PL-NAc, $n=6$ mice, and mTH-NAc, $n=9$ mice).

In contrast to the preferential representation of choice in PL-NAc compared to mTH-NAc, there was a larger fraction of neurons in mTH-NAc that encoded outcome compared to PL-NAc (Figure 3c; mTH-NAc: 85/256 (33\%), PL-NAc: 70/278 (25\%); P=0.038: two-proportion Z-test; significant outcome-selectivity was determined using a nested comparison of the encoding model with and without outcome information, see Methods). However, while outcome decoding accuracy in mTH-NAc was slightly better relative to PL-NAc (Figure 3d; mTH-NAc: $73 \pm 2 \%$, PL-NAc: $69 \pm 1 \%$ ), this difference was not statistically significant $(P=0.11$ :unpaired, two-tailed t-test comparing peak decoding accuracy across mice between PL-NAc ( $n=6$ mice) and $m$ TH-NAc $(n=9$ mice)). These results suggest that, unlike the preferential choice representation observed in PL-NAc over mTH-NAc, outcome was more similarly represented between these two populations. This is presumably due to the fact that both CS+ and reward consumption responses contribute to outcome representation, and although more neurons encoded CS+ in $\mathrm{mTH}-\mathrm{NAc}$, the opposite was true for reward consumption (Figure 2c,d).

To determine if choice or outcome decoding in either population depended on recording location, we aligned GRIN lens tracks from the histology to the Allen atlas (see Methods). We found no relationship between the strength of either choice or outcome decoding and recording location in either PL-NAc or mTH-NAc (Supplementary Figure 4).

\section{PL-NAc neurons display choice-selective sequences that persist into the next trial}

We next examined the temporal organization of choice-selective activity in PL-NAc neurons. Across the population, choice-selective PL-NAc neurons displayed sequential activity with respect to the lever press that persisted for $>4 \mathrm{~s}$ after the press. These sequences were visualized by time-locking the GCaMP6f fluorescence of choice-selective neurons with respect to the lever press, rather than with the encoding model from the earlier figures (Figure 4a-c; see Supplementary Figure 5 for sequences without peak-normalization). The robustness of these sequences was confirmed using a cross-validation procedure, in which the order of peak activity across the PL-NAc choice-selective population was first established using half the trials (Figure $\mathbf{4 b}$, 'train'), and then the population heatmap was plotted using the same established ordering and activity from the other half of trials (Figure 4c, 'test'). To quantify the consistency of these sequences, we correlated the neurons' time of peak activity in the 'training' and 'test' data, and observed a strong correlation (Figure 4d; $\mathrm{R}^{2}=0.81, \mathrm{P}=6.4 \times 10^{-23}, \mathrm{n}=92$ neurons from 7 mice). Additionally, the ridge-to-background ratio, a metric used to confirm the presence of sequences (see (Akhlaghpour et al., 2016; Harvey et al., 2012; Kondo et al., 2017)) was significantly higher when calculated using the PL-NAc choice-selective sequences compared with sequences generated using shuffled data (Supplementary Figure 6a-c; $P<0.001$, t-test between ratio calculated from unshuffled data and 500 iterations of shuffled data).

In contrast, choice-selective sequential activity in the mTH-NAc population was significantly less consistent than in PL-NAc (Supplementary Figure 7a-d; $Z=2.34, P=0.0096$ : Fisher's $Z$, comparison of correlation coefficients derived from comparing peak activity between 'test' and 'training' data from PL-NAc versus mTH-NAc). Additionally, while the ridge-to-background ratio of the sequences generated using $\mathrm{mTH}-\mathrm{NAc}$ activity was significantly higher than that using shuffled data (Supplementary Figure 6d-f; $\mathrm{P}<0.001$ ) this ratio was also significantly lower than that obtained from PL-NAc sequences $(2.36+/-0.12$ mean+/-sem ratio for choice-selective $m T H-N A c$ neurons, $n=42$, vs 3.01+/-0.12 mean+/-sem ratio for choice-selective $\mathrm{PL-NAc}, \mathrm{n}=92 ; \mathrm{P}=0.001$ : unpaired, two-tailed t-test comparing ratio between $\mathrm{PL}-\mathrm{NAc}$ and $\mathrm{mTH}-\mathrm{NAc}$ neurons).

A striking feature of these choice-selective sequences in PL-NAc was that they persisted for seconds after the choice, potentially providing a neural 'bridge' between action and outcome. To 
further quantify the timescale of choice encoding, both within and across trials, we used activity from simultaneously imaged neurons at each timepoint in the trial to predict the mouse's choice (with a decoder based on a logistic regression using random combinations of 10 simultaneously imaged neurons to predict choice). Choice on the current trial could be decoded above chance for $\sim 7 \mathrm{~s}$ after the lever press, spanning the entire trial (including the time of reward delivery and consumption), as well as the beginning of the next trial (Figure 4e; $P<0.01$ : unpaired, two-tailed t-test of decoding accuracy across mice at the time of the next trial nose poke compared with chance decoding of 0.5 , $\mathrm{n}=6$ mice). Choice on the previous or subsequent trial was not represented as strongly as current trial choice (Figure 4e; in all cases we corrected for cross-trial choice correlations with a weighted decoder, see Methods) and choice from two trials back could not be decoded above chance at any time point in the trial (Supplementary Figure 8). We also examined the temporal extent of choice encoding in the mTH-NAc population (Supplementary Figure 7e). Similar to PL-NAc, we observed that decoding of the mice's choice persisted up to the start of the next trial. However, the peak decoding accuracy across all time points in the trial was lower in mTH-NAc $(59 \% \pm 0.2 \%)$ compared with PL-NAc (73 $\pm 0.2 \%)$.

Choice-selective sequences in PL-NAc neurons, in combination with known anatomy, can provide a substrate for temporal difference (TD) learning

Thus far, we observed that choice-selective sequences in PL-NAc neurons encoded the identity of the chosen lever for multiple seconds after the lever press. This sequential activity bridged the gap in time between a mouse's action and reward feedback, and therefore contained information that could be used to solve the task (Figure 4c,e). But how could a biologically realistic network use sequences to implement this task? In particular, how could PL-NAc neurons (and their synapses) that were active at the initiation of the choice-selective sequence - and could be important to generating the appropriate sequence - be strengthened by an outcome that occurred toward the end of the sequence?

To address this question, we developed a circuit-based computational model that could perform this task based on the observed choice-selective sequences in PL-NAc neurons. This was achieved using a model that implemented a temporal difference (TD) reinforcement learning algorithm by combining the recorded choice-selective sequential activity of PL-NAc neurons with the known connectivity of downstream structures (Figure $\mathbf{5 a , b}$ ). The goal of TD learning is for weights to be adjusted in order to predict the sum of future rewards, or "value", as well as possible (Dayan and Niv, 2008; O'Doherty et al., 2003; Sutton and Barto, 1998; Tsitsiklis and Van Roy, 1997). When this sum of future rewards changes, such as when an unexpected reward is received or an unexpected predictor of reward is experienced, a TD reward prediction error (RPE) occurs and adjusts the weights to reduce this error. The error signal in the TD algorithm closely resembles the RPE signal observed in ventral tegmental area (VTA) dopamine neurons (Parker et al., 2016; Schultz, 1998; Schultz et al., 1997), but how this signal is computed remains an open question.

In our model, the PL-NAc sequences enabled the calculation of the RPE in dopamine neurons. Over the course of a series of trials, this error allowed a reward that occurred at the end of the PL-NAc sequence to adjust synaptic weights at the beginning of the sequence (Figure 5a,b). Our model generated an RPE in dopamine neurons based on minimal assumptions: i) the choice-selective sequences in PL-NAc neurons that we report here (Figure 5c), ii) established (but simplified) anatomical connections between PL, NAc, and VTA neurons (see circuit diagram in Figure 5a), and iii) dopamine- and activity-dependent modification of the synapses connecting PL and NAc neurons (with a synaptic eligibility decay time constant of $0.6 \mathrm{~s}$, consistent with (Gerstner et al., 2018) and (Yagishita et al., 2014)). In addition to the proposed circuit architecture of Figure 5a, we present several variant circuits, which also are able to calculate an RPE signal using the recorded choice-selective PL-NAc sequences (Supplementary Figure 9). 
In more detail, our model took as inputs experimental, single-trial recordings of choice-selective, sequentially active PL neurons (Figure 5a, see Methods). These inputs represented temporal basis functions ( $f_{i}(t)$ in Figure $\mathbf{5 a}$ ) for computing the estimated value of making a left or right choice. These basis functions are weighted in the NAc by the strength $w_{i}$ of the PL-NAc synaptic connection and summed together in the ventral pallidum (VP) to create a (sign-inverted) representation of the estimated value, at time $t$, of making a left choice, $V_{L}(t)$, or right choice, $V_{R}(t)$. To create the RPE observed in DA neurons requires that the DA neuron population receive a fast, positive value signal $V(t)$ and a delayed negative value signal $V(t-\Delta)$, as well as a direct reward signal (Figure 5b). In our model, the fast value signal is due to direct VP to DA input. The delayed negative signal to the DA population is due to a slower, disynaptic pathway that converges first upon the VTA GABA neurons, so that these neurons encode a value signal as observed experimentally (Eshel et al., 2015). The temporal discounting factor $\gamma$ is implemented through different strengths of the two pathways to the VTA DA neurons (Figure 5b).

Learning is achieved through DA-dependent modification of the PL-NAc synaptic strengths. We assume that PL-NAc neuronal activity leads to an exponentially decaying "eligibility trace" (Gerstner et al., 2018; Sutton and Barto, 1998). The correlation of this presynaptically driven eligibility trace with DA input then drives learning (Figure 5b). Altogether, this circuit architecture (as well as the mathematically equivalent architectures shown in Supplementary Figure 9) realizes a temporal difference learning algorithm for generating value representations in the ventral basal ganglia, providing a substrate for the selection of proper choice based on previous trial outcomes. The TD model was able to correctly perform the task and recapitulate the mice's behavior, achieving a comparable rate of reward $(47.5 \%$ for the model versus $47.6 \%$ for the mice). Similar to mice, the model alternated choice following block transitions (Figure 5d,e; compare to Figure 1b,c; choice based upon a probabilistic readout of the difference between right and left values at the start of the sequence, see Methods) and had a higher stay probability following rewarded trials relative to unrewarded trials (Figure 5f; compare to Figure 1d).

Appropriate task performance was achieved through the calculation of an RPE signal in VTA dopamine neurons. The RPE signal was evident within a trial, based on the positive response to rewarded outcomes and negative response to unrewarded outcomes (Figure $\mathbf{5 g}$, top). The RPE signal was also evident across trials, based on the negative modulation of the dopamine outcome signal by previous trial outcomes (Figure $\mathbf{5 g}$, bottom, multiple linear regression similar to (Bayer and Glimcher, 2005; Parker et al., 2016).

Several features of the model neuron responses resembled those previously observed experimentally. The model dopamine responses were similar to results obtained from recorded dopamine activity in the same task ((Parker et al., 2016); Supplementary Figure 10). The VTA GABA interneuron had a sustained value signal, due to the converging input of the transient, sequential value signals from NAc/VP (Supplementary Figure 11), replicating the sustained value signal in VTA GABA interneurons recently found in monosynaptic inputs to the VTA dopamine neurons (Cohen et al., 2012). We note that, alternatively, the VP neurons shown in Figure 5a could project to a second set of VP neurons that functionally take the place of the VTA GABA interneurons (Supplementary Figure 9d), leading to sustained positive value encoding VP neurons as were recently observed in VTA -projecting VP neurons (Tian et al., 2016). Additionally, the convergence of the right-choice and left-choice neurons could occur in VP rather than VTA, so that there is no explicit encoding of $V_{L}$ and $V_{R}$ (Supplementary Figure 9d).

We next asked how the same model using single-trial activity from choice-selective mTH-NAc neurons would perform (Figure $5 \mathrm{~h}$ ). In line with the less consistent sequential choice-selective activity in mTH-NAc relative to PL-NAc (Figure 3a,b, Figure 4d \& Supplementary Figure 7d), we observed a substantial reduction in performance when using mTH-NAc activity as input (Figure 5i-k). In fact, the mTH-NAc model performed at chance levels (39.7\% reward rate, compared with chance reward 
rate of $40 \%$ ). Additionally, relative to the PL-NAc model, using mTH-NAc activity resulted in a reduction in the negative modulation of dopamine signal following an unrewarded outcome (Figure 5I, top; compare with Figure 5g, top), and less effect of previous trial outcome on dopamine response (Figure 5I, bottom; compare with Figure 5g, bottom). Compared with PL-NAc, using mTH-NAc as input also resulted in a reduction in the speed at which the correct value is learned within the NAc and VTA GABA neurons (Supplementary Figure 11c,d; compare with Supplementary Figure 11a,b).

The choice-selective sequences in PL-NAc neurons were critical to model performance, as they allowed the backpropagation of the RPE signal across trials. This was verified by comparing the performance of the model to a model in which PL-NAc activity was shifted to be synchronously active at the trial onset (Figure $5 \mathrm{~m}$ ). Unlike the sequential data, synchronous choice-selective PL-NAc activity was unable to correctly modulate lever value following block transitions, and therefore did not lead to correct choices, resulting in model performance near chance (Figure 5n-p; synchronous model reward rate: $39.8 \%$, compared with chance reward rate of $40 \%$ ). This was due to the fact that the synchronous model was unable to generate an RPE signal that was modulated properly within or across trials. Within a trial, negative outcomes did not result in the expected decrease in the dopamine signal (Figure 5q), while across trials, the influence of the previous trial's outcome on the dopamine signal was disrupted relative to both the sequential model and recorded dopamine activity (compare to Figure $\mathbf{5 g}$ and recorded dopamine activity in NAc in Supplementary Figure 10). Without a properly calculated RPE signal, the synchronous model was unable to generate value signals that correlated with the identity of the high probability lever in either NAc or the VTA GABA interneuron (Supplementary Figure 11e,f).

Stimulation of PL-NAc (but not mTH-NAc) neurons decreases the effect of previous trial outcomes on subsequent choice in both the model and the mice

We next sought to generate experimentally testable predictions from our TD model simulation by examining the effect of disruption of these sequences on behavioral performance. Towards this end, we simulated optogenetic-like neural stimulation of this projection by replacing the PL-NAc sequential activity in the TD model with constant, population-wide and choice-independent activity across the population on a subset of trials (on $10 \%$ of trials, $65 \%$ of neurons were stimulated; Figure $\mathbf{6 a}, \mathbf{b})$. This generated a decrease in the probability of staying with the previously chosen lever following rewarded trials and an increase following unrewarded trials relative to unstimulated trials (Figure 6c). In other words, the effect of previous outcome on choice was reduced when PL-NAc activity was disrupted. This effect persists for multiple trials, as revealed by including terms that account for the interaction between previous rewarded and unrewarded choices and stimulation in the logistic choice regression introduced in Figure 1e (Figure 6d; see Methods for details). This occurs because stimulation disrupts the choice-selectivity that is observed in the recorded PL-NAc sequences, thus allowing dopamine to indiscriminately adjust the synaptic weights (i.e. value) of both the right and left PL-NAc synapses following rewarded or unrewarded outcomes. The effect of stimulation is observed multiple trials back because the incorrect weight changes persist for multiple trials in the TD model. In contrast, stimulation did not result in a difference in stay probability on the trial with stimulation, as choice was determined by spontaneous activity before the sequence initiates in PL-NAc neurons, combined with the synaptic weights between PL and NAc neurons (Figure 6c).

We tested these model predictions experimentally by performing an analogous manipulation in mice, which involved activating PL-NAc axon terminals with ChR2 on $10 \%$ of trials (Figure 6e). In close agreement with our TD model, mice had a significant decrease in their stay probability following a rewarded trial that was paired with stimulation (Figure 6f; $P=0.001$ : paired, two-tailed t-test across mice, $n=14$, comparison between stay probability following rewarded trials with and without stimulation), while they were more likely to stay following an unrewarded trial paired with stimulation (Figure 6f; $P=0.0005$ : paired, two-tailed t-test across mice, $n=14$, comparison between stay 
probability following unrewarded trials with and without stimulation). Similar to the TD model (Figure $\mathbf{6 d}$ ), the effect of stimulation on the mouse's choice persisted for multiple trials. Mice had a significant decrease in their stay probability following PL-NAc stimulation on rewarded choices one and two trials back (Figure 6g; $P=0.001$ for one trial back; $P=0.02$ for two trials back; one-sample, two-tailed t-test across mice of regression coefficients corresponding to the interaction term between rewarded choice and optical stimulation, $\mathrm{n}=14$ mice).

Also similar to the model, and in contrast to these effects on the next trial, stimulation on the current trial had no significant effect on choice following either rewarded or unrewarded trials (Figure 6f; $P>0.5$ : paired, two-tailed t-test across mice, $n=14$, comparison between stay probability on trials with and without stimulation following both rewarded and unrewarded trials).

We also observed an increase in the probability of mice abandoning the trials with stimulation compared with those trials without, suggesting that this manipulation had some influence on the mouse's motivation to perform the task $(P=0.0006$ : paired, two-tailed t-test comparing percentage of abandoned trials on activated versus non-activated trials; $12.2+/-2.5 \%$ for activated trials, $0.9+/-0.2 \%$ for non-activated trials).

Given the relatively weak choice encoding in mTH-NAc compared to PL-NAc (Figure 3a,b), and the fact that the mTH-NAc did not support effective trial-by-trial learning in our model (Figure $5 \mathrm{~m}-\mathrm{q}$ ), we hypothesized that optogenetic stimulation of the mTH-NAc projection might not impact choice (Figure 6h). Indeed, in contrast to PL-NAc stimulation, mTH-NAc stimulation had no significant effect on the mice's stay probability on the subsequent trial, following either rewarded or unrewarded stimulation trials (Figure 6i; $P=0.84$ : paired, two-tailed t-test across mice, $n=8$, comparison of stay probability following rewarded trials with and without stimulation; $\mathrm{P}=0.40$ : paired two-tailed t-test, comparison of stay probability following unrewarded trials with and without stimulation). Similarly, inclusion of mTH-NAc stimulation into our choice regression model from Figure 1e revealed no significant effect of stimulation on rewarded or unrewarded choices (Figure $6 \mathbf{j}$; $P>0.05$ for all trials back: one-sample, two-tailed t-test of regression coefficients corresponding to the interaction term between rewarded or unrewarded choice and optical stimulation across mice, $n=8$ mice). Additionally, there was no effect on the mice's stay probability for current trial stimulation (Figure 6i; $P=0.59$ : paired, two-tailed t-test across mice, $n=8$, comparison of stay probability following rewarded trials with and without stimulation; $P=0.50$ : paired, two-tailed t-test, comparison of stay probability following unrewarded trials with and without stimulation). Similar to PL-NAc stimulation, mTH-NAc stimulation generated an increase in the probability of abandoning a trial on stimulation trials compared with control trials $(\mathrm{P}=0.032$ : paired, two-tailed t-test comparing percentage of abandoned trials on activated versus non-activated trials; $22.1+/-7.9 \%$ for activated trials, $6.4+/-3.1 \%$ for non-activated trials), indicating that laser stimulation of the mTH-NAc projection may affect motivation to perform the task while not affecting the mouse's choice.

To control for non-specific effects of optogenetic stimulation, we ran a control cohort of mice that received identical stimulation but did not express the opsin (Supplementary Figure 12a,b). Stimulation had no effect on the mice's choice behavior (Supplementary Figure 12c,d) nor on the probability of abandoning trials on stimulation versus control trials $(\mathrm{P}=0.38$ : paired, two-tailed t-test comparing percentage of abandoned trials on activated versus non-activated trials; $0.4+/-0.08 \%$ for activated trials, $0.4+/-0.01 \%$ for non-activated trials).

\section{Discussion}

This work provides both experimental and computational insights into how the NAc and associated regions could contribute to reinforcement learning. Experimentally, we found that mTH-NAc neurons are preferentially modulated by a reward-predictive cue, while PL-NAc neurons more strongly encoded actions (e.g. nose poke, lever press). In addition, PL-NAc neurons display 
choice-selective sequential activity which persists for several seconds after the lever press action, beyond the time the mice receive reward feedback. Computationally, we demonstrate that the choice-selective and sequential nature of PL-NAc activity can contribute critically to performance of a choice task by implementing a circuit-based version of TD learning (Sutton and Barto, 1998; Tesauro, 1992). Despite its simplicity, the model is able to i) perform the task, ii) replicate previous recordings in VTA dopamine and GABA neurons, and iii) make new predictions that we have experimentally tested regarding the effect of perturbing PL-NAc or mTH-NAc activity on trial-by-trial learning. Thus, this work suggests a computational role of choice-selective sequences, a form of neural dynamics whose ubiquity is being increasingly appreciated (Kawai et al., 2015; Kim et al., 2017; Long et al., 2010; Ölveczky et al., 2011; Picardo et al., 2016; Sakata et al., 2008).

\section{Relationship to previous neural recordings in the NAc and associated regions}

To our knowledge, a direct comparison, at cellular resolution, of activity across multiple glutamatergic inputs to the NAc has not previously been conducted. This is a significant gap, given that these inputs are thought to contribute critically to reinforcement learning by providing the information to the NAc that dopaminergic inputs can modulate (Centonze et al., 2001; Nestler, 2001; Nicola et al., 2000; Shen et al., 2008; Wilson, 2004; Xiong et al., 2015). The differences in the representations in the two populations that we report were apparent due to the use of a behavioral task with both actions and sensory stimuli.

The preferential representations of actions relative to sensory stimuli in PL-NAc is somewhat surprising, given that previous studies have focused on sensory representations in this projection (Otis et al., 2017), and also given that the NAc is heavily implicated in Pavlovian conditioning (Day and Carelli, 2007; Day et al., 2006; Di Ciano et al., 2001; Parkinson et al., 1999; Roitman et al., 2005; Wan and Peoples, 2006). On the other hand, there is extensive previous evidence of action correlates in PFC (Cameron et al., 2019; Genovesio et al., 2006; Luk and Wallis, 2013; Siniscalchi et al., 2019; Sul et al., 2010), and NAc is implicated in operant conditioning in addition to Pavlovian conditioning (Atallah et al., 2007; Cardinal and Cheung, 2005; Collins et al., 2019; Hernandez et al., 2002; Kelley et al., 1997; Kim et al., 2009; Salamone et al., 1991).

Our finding of sustained choice-encoding in PL-NAc neurons is in agreement with previous work recording from medial prefrontal cortex (mPFC) neurons during a different reinforcement learning task (Maggi and Humphries, 2019; Maggi et al., 2018). Additionally, other papers have reported choice-selective sequences in other regions of cortex, as well as in the hippocampus (Harvey et al., 2012; Pastalkova et al., 2008; Terada et al., 2017). In fact, given previous reports of choice-selective (or outcome-selective) sequences in multiple brain regions and species (Kawai et al., 2015; Kim et al., 2017; Long et al., 2010; Ölveczky et al., 2011; Picardo et al., 2016; Sakata et al., 2008), the relative absence of sequences in mTH-NAc neurons may be more surprising than the presence in PL-NAc.

Our observation of prolonged representation of the CS+ in mTH-NAc (Figure 2d) is in alignment with previous observations of pronounced and prolonged encoding of task-related stimuli in the primate thalamus during a Pavlovian conditioning task (Matsumoto et al., 2001). Together with our data, this suggests that the thalamus is contributing information about task-relevant stimuli to the striatum, which is likely critical for Pavlovian conditioning (Campus et al., 2019; Do-Monte et al., 2017; Otis et al., 2019; Zhu et al., 2018).

\section{Choice-selective sequences implement TD learning in a choice task}

Given the widespread observation of choice-selective sequences across multiple behaviors and brain regions, including the PL-NAc neurons that we record from in this study, a fundamental question is what is the computational function of such sequences. Here, we suggest that these 
sequences may contribute to the neural implementation of TD learning, by providing a temporal basis set that bridges the gap in time between actions and outcomes. Specifically, neurons active in a sequence that ultimately results in reward enable the backpropagation in time of the dopaminergic RPE signal, due to the fact that the earlier neurons in the sequence predict the activity of the later neurons in the sequence, which themselves overlap with reward. This causes synaptic weights onto NAc of these earlier neurons to be strengthened, which in turn biases the choice towards that represented by those neurons.

While TD learning based on a stimulus representation of sequentially active neurons has previously been proposed for learning in the context of sequential behaviors (Fee and Goldberg, 2011; Jin et al., 2009), and for learning the timing of a CS-US relationship (Aggarwal et al., 2012; Carrillo-Reid et al., 2008; Gershman et al., 2014; Ponzi and Wickens, 2010), here we extend these ideas in several important ways. First, we link these theoretical ideas directly to data, by demonstrating that choice-selective sequential activity in the NAc is provided primarily by PL-NAc (as opposed to mTH-NAc) input neurons, and that perturbation of the PL-NAc (but not mTH-NAc) projection disrupts action-outcome pairing consistent with model predictions. Specifically, in the model, overwriting the choice-selective sequential activity with full trial optogenetic-like stimulation of PL-NAc neurons disrupts the model's ability to link choice and outcome on one trial to guide choice on the subsequent trials (Figure 6b-d). In contrast to the effect of stimulation on subsequent trials' choice, stimulation causes no disruption of choice on the stimulated trial, in either the model or the experiment (Figure 6c,f). This is true in the model because choice is determined by the PL-NAc weights at the beginning of the trial, which are determined by previous trials' choices and outcomes. Thus, the model provides a mechanistic explanation of a puzzling experimental finding: that optogenetic manipulation of PL-NAc neurons affects subsequent choices but not the choice on the stimulation trial itself and that this stimulation creates oppositely directed effects following rewarded versus unrewarded trials.

Second, we extend these ideas to the performance of a choice task. For appropriate learning in a choice task, an RPE must update the value of the chosen action only. Given that dopamine neurons diffusely project to striatum, and given that the dopamine RPE signal is not action-specific (Lee et al., 2019), it is not immediately obvious how such specificity would be achieved. In our model, the specificity of the value updating by dopamine to the chosen action is possible because the PL-NAc neurons that are active for one choice are silent for the other choice. Similarly, even though dopamine neurons receive convergent inputs from both NAc/VP neurons that encode right-side value as well as others that encode left-side value (given, once again, that dopamine RPE signals are not action-specific), the model produces dopamine neuron activity that is dependent on the value of the chosen action (rather than the sum of left and right action values) by virtue of the fact that PL-NAc inputs that are active for one choice are silent for the other. Thus, a key feature of choice-selective sequences that is critical to our model is that neurons that are active for one choice are silent for the other choice.

Third, our model replicates numerous experimental findings in the circuitry downstream of PL-NAc. The most obvious is the calculation of an RPE signal in dopamine neurons (Bayer and Glimcher, 2005; Parker et al., 2016), which allows proper value estimation and, thus, task performance. We had previously confirmed an RPE signal in NAc-projecting dopamine neurons in this task (Parker et al., 2016). In addition, GABA interneurons encode value (Cohen et al., 2012; Tian et al., 2016), as predicted by our model, and their activation inhibits dopamine neurons, again consistent with our model (Eshel et al., 2015). This value representation in the GABA interneurons is key to our model producing an RPE signal in dopamine neurons, as it produces the temporally delayed, sign inverted signals required for the calculation of a temporally differenced RPE (Figure 5a). Although previous work had suggested other neural architectures for this temporal differencing operation (Aggarwal et al., 2012; Carrillo-Reid et al., 2008; Doya, 2002; Hazy et al., 2010; Ito and Doya, 2015; Joel et al., 2002; Pan et al., 2005; Suri and Schultz, 1998, 1999), these models have not been 
revisited in light of recent cell-type and projection-specific recordings in the circuit. Consistent with our model, electrical stimulation of VP generates both immediate inhibition of dopamine neurons, and delayed excitation, as required by our model (Chen et al., 2019).

Our specific proposal for temporal differencing by the VTA GABA interneuron is attractive in that it could provide a generalizable mechanism for calculating RPE: it could extend to any input that projects both to the dopamine and GABA neurons in the VTA, and that also receives a dopaminergic input that can modify synaptic weights.

\section{Evidence that dopamine and synaptic plasticity contribute to reversal learning}

In our model, appropriate trial-and-error learning in our probabilistic reversal learning task is mediated by a dopamine-dependent synaptic plasticity mechanism. There is extensive evidence to support the development of a reinforcement learning model for this task. For example, we had previously demonstrated that dopamine neurons that project to the NAc encode an RPE signal in this task, and that inhibiting these neurons serves as a negative prediction error signal, consistent with a reinforcement learning mechanism (Parker et al., 2016). Similar evidence for dopamine serving as a reinforcement learning signal has been obtained in other related paradigms in both rodents (Hamid et al., 2016; Kwak et al., 2014; Lak et al., 2020) and human subjects (Rutledge et al., 2009). In addition, other studies have used pharmacology or ablation to implicate dopamine-receptor pathways and NMDA-receptors in NAc in reversal learning (Boulougouris et al., 2009; Ding et al., 2014; Izquierdo et al., 2006; Kruzich and Grandy, 2004; O’Neill and Brown, 2007; Taghzouti et al., 1985).

Another mechanism aside from dopamine and synaptic plasticity that could contribute to performing a trial-and-error learning task is working memory. In fact, there is evidence that mice may use both reinforcement learning and working memory in parallel to perform a wide range of tasks (Collins and Frank, 2012; Collins et al., 2014, 2017). Indeed, in our TD model, we include a stay bias term (see Methods for details), which increases the probability of returning to the previously chosen lever and would require a sustained identity of choice for a single trial-potentially through a short-term memory mechanism. In principle, this may be mediated by the sustained choice representation observed in our PL-NAc recordings. However, our results from PL-NAc optogenetic stimulation (Figure $\mathbf{6 g}, \mathbf{h}$ ) suggest that this projection may not mediate this bias, as stimulation has an opposite effect on choice after rewarded and unrewarded trials. In contrast to these results, the bias term causes mice to repeat the previous choice irrespective of previous trial outcome.

A critical component of a reinforcement learning model of trial-and-error learning is the ability of dopamine to modulate synaptic strength on a timescale that matches that of the observed behavior-in the case of the present study, on the order of a single trial or tens of seconds. (Figure 1c-e). While much of the work regarding dopamine and synaptic plasticity has not focused on the timescale of induction of LTP or LTD, there is evidence that dopamine is able to regulate synaptic weights on a rapid timescale. For example, recent work has demonstrated that D1-MSNs display robust, sustained changes in their firing rate within hundreds of milliseconds of transient dopamine terminal stimulation (Lahiri and Bevan, 2020). Additionally, foundational experiments have shown robust increases in the strength of postsynaptic potentials at corticostriatal synapses immediately after an ICSS stimulation protocol (Reynolds et al., 2001). These results are supported by the observation that brief optogenetic activation of dopamine striatal terminals are able to rapidly (on the order of seconds) modulate CaMKII activation as well as spine morphology, processes critically involved in the formation of long term synaptic plasticity (Yagishita et al., 2014). Furthermore, dopamine has been shown to play a critical role in forms of synaptic plasticity that operate on the time-scale of milliseconds to seconds, such as facilitation and short-term depression, at both excitatory and inhibitory inputs to NAc medium spiny neurons (Hjelmstad, 2004; Jayasinghe et al., 2017; Tecuapetla et al., 2007). 
Potential extensions of our model

Consistent with the observation that $\mathrm{mTH}-\mathrm{NAc}$ displayed weaker choice representation relative to PL-NAc, disruption of this $\mathrm{mTH}$ projection did not have a causal effect on our choice task (Figure 3a,b; Figure $6 \mathbf{h}-\mathbf{j}$ ). Given the strong and prolonged outcome encoding observed in this population (Figure 2d,f; Figure 3c,d), it is possible the mTH-NAc projection would instead be relevant had the goal of the task been to bridge a delay between a CS and a US (i.e. a pavlovian trace conditioning task rather than an operant task).

While our model replicates multiple features of neurons throughout the circuit, it does not predict the relative timing and sign of the value correlates in the NAc versus the VP (Chen et al., 2019; Ottenheimer et al., 2018; Tian et al., 2016), likely because of anatomical connections that we omitted from our circuit for simplicity (e.g.VP $\rightarrow$ NAc, (Ottenheimer et al., 2018; Wei et al., 2016); also see Supplementary Figure $\mathbf{9}$ for discussion of feasible model variants that produce a greater diversity of signals in VP). Similarly, our model does not produce previously observed responses to the conditioned stimuli in VP (Ottenheimer et al., 2019; Stephenson-Jones et al., 2020; Tian et al., 2016; Tindell et al., 2004), although inclusion of cue-responsive mTH-NAc inputs into our model would remedy this.

Another element of the circuit that we did not include in this model is the indirect feedback loops from the basal ganglia back to mPFC. Inclusion of this feedback would produce representations of value and not just choice in mPFC, consistent with previous observations (Bari et al., 2019; Gläscher et al., 2009; Grabenhorst and Rolls, 2011; Kim et al., 2008). These value representations may bias choice via the "spiraling out" architecture of the basal ganglia (Haber and Knutson, 2010; Haber et al., 2000; Ikeda et al., 2013; Nauta et al., 1978), which could allow value correlates in NAc to ultimately influence action implementation in more dorsal regions like ALM, that project to motor outputs. Note that inclusion of such feedback would produce representations of value and not just choice in more dorsal regions of mPFC/ACC/M2 (Bari et al., 2019; Gläscher et al., 2009; Grabenhorst and Rolls, 2011; Kim et al., 2008). In addition, the indirect feedback from the NAc back to mPFC could also contribute to the formation of the PL sequences in the first place, with dopaminergic modulation of NAc activity at one time point helping to trigger changes in cortical activity at the next time point. Further experiments and model extensions will be needed to explore these ideas. 
bioRxiv preprint doi: https://doi.org/10.1101/725382; this version posted July 17,2020 . The copyright holder for this preprint (which was not certified by peer review) is the author/funder. All rights reserved. No reuse allowed without permission.

\section{Figures}

a

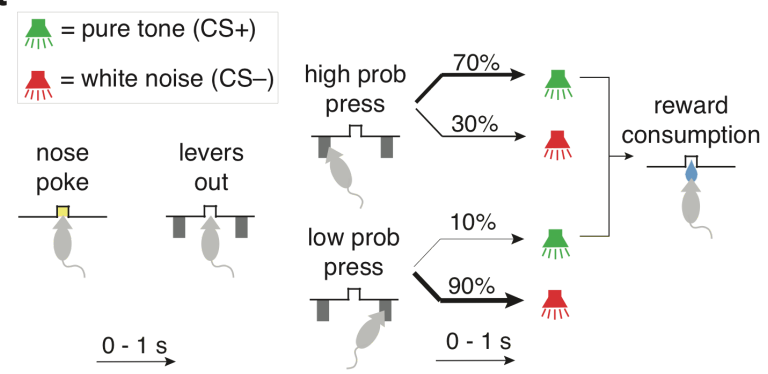

b
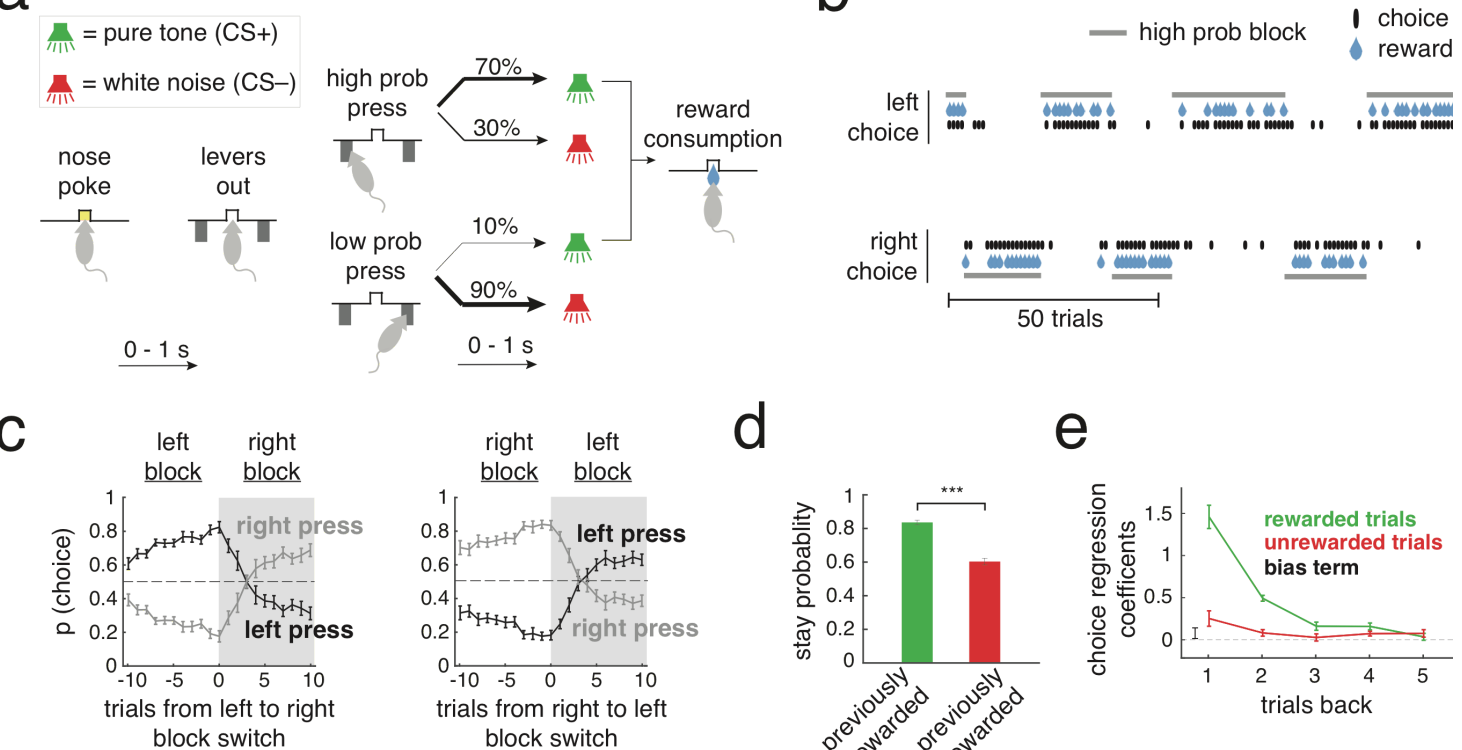

block switch

f
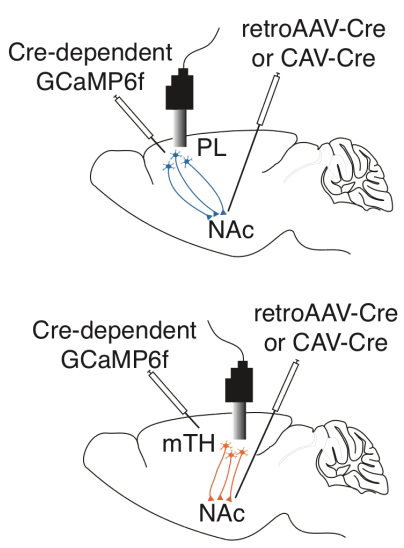

e

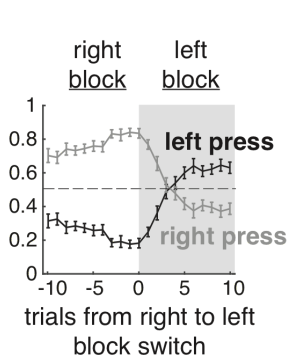

d
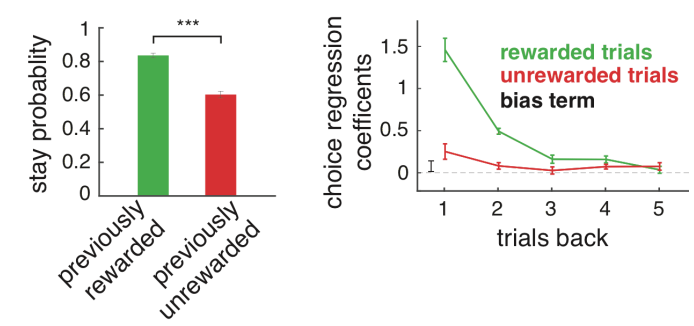

g
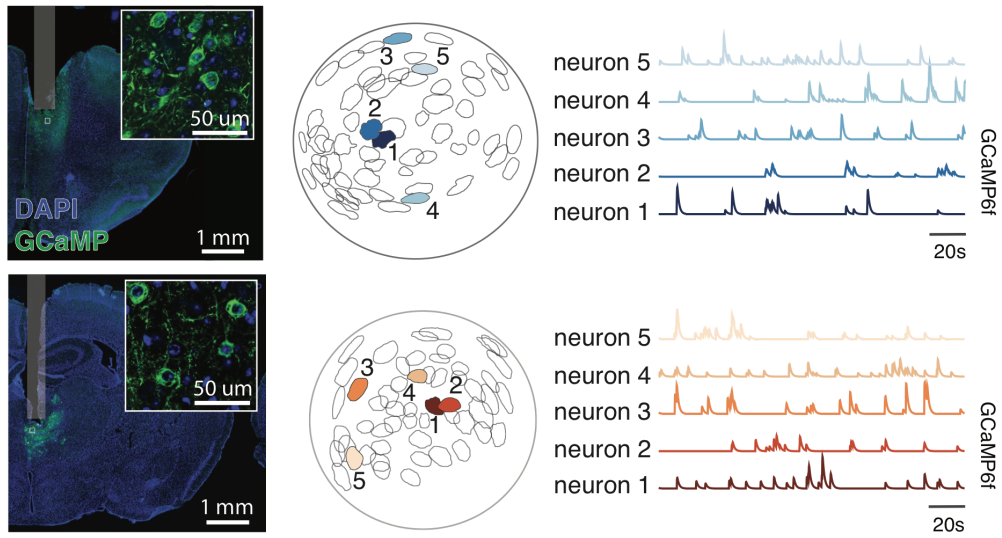

Figure 1 - Cellular-resolution imaging of PL and $\mathrm{mTH}$ neurons that project to the NAc in mice performing a reinforcement learning task. (a) Schematic of probabilistic reversal learning task. Mice began a trial by entering a central nose poke ('nose poke') which resulted in the presentation of two levers ('levers out'). A lever press resulted in either a rewarded (CS+, reward consumption) or unrewarded (CS-) outcome, with the probability of a given outcome dependent on whether the trial was in a right or left high-probability block. A high probability press (i.e. a left press in a left block, or a right press in a right block) led to reward on $70 \%$ of trials, while a low-probability press led to reward on 10\%. (b) Example behavior of a mouse performing 120 trials during a recording session. The choice of the mouse (black marks) follows the identity of the higher probability lever as it alternates between a left and right block (horizontal grey bars). (c) Left, Probability of the mice choosing either the left or right lever 10 trials before and after a switch from a left to right high-probability block. Right, same as left except choice probabilities following a right to left high-probability block. (d) Mice had a significantly higher stay probability following a rewarded versus unrewarded trial (*** $p=5 \times 10^{-9}$, two-tailed t-test, $n=16$ mice). (e) Coefficients from a logistic regression that uses choice and outcome from the previous five trials to predict choice on the current trial. The regression used two sets of predictors: (i) 'Rewarded trials' (green) which identify a previous trial as a rewarded right press $(+1)$, rewarded left press $(-1)$ or unrewarded press $(0)$ and (ii) 'Unrewarded trials' (red), which identify a previous trial as an unrewarded right press $(+1)$, unrewarded left press $(-1)$ or rewarded trial $(0)$. Positive regression coefficients correspond to a greater likelihood of the mouse making a lever press with the same choice identity as that of the previous trial. Error bars in c,d,e represent s.e.m. across mice $(n=16)$. (f) Left, surgical schematic for PL-NAc (top) and mTH-NAc (bottom) recordings showing the injection site and optical lens implant with miniature head-mounted microscope attached. Right, Coronal section from a PL-NAc (top) and mTH-NAc (bottom) mouse showing GCaMP6f expression in each respective recording site. Inset: confocal image showing GCaMP6f expression in individual neurons. (g) Left, example field of view from a recording in PL-NAc (top, blue) and mTH-NAc (bottom, orange) with five representative regions of interest (ROIs). Right, normalized GCaMP6f fluorescence traces from the five ROls on the left. For visualization purposes, each trace was normalized by the peak fluorescence across the hour long session. 
bioRxiv preprint doi: https://doi.org/10.1101/725382; this version posted July 17,2020 . The copyright holder for this preprint (which was not certified by peer review) is the author/funder. All rights reserved. No reuse allowed without permission.

a
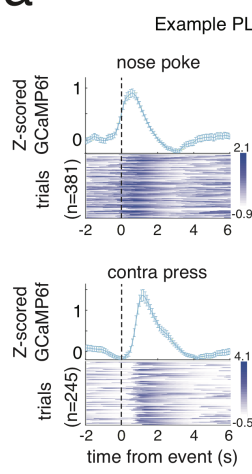

C

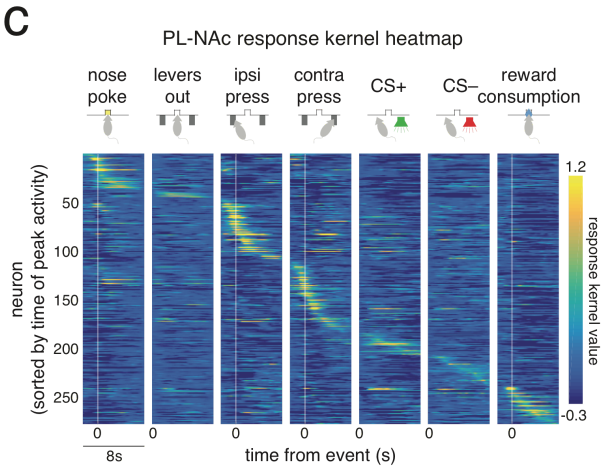

e

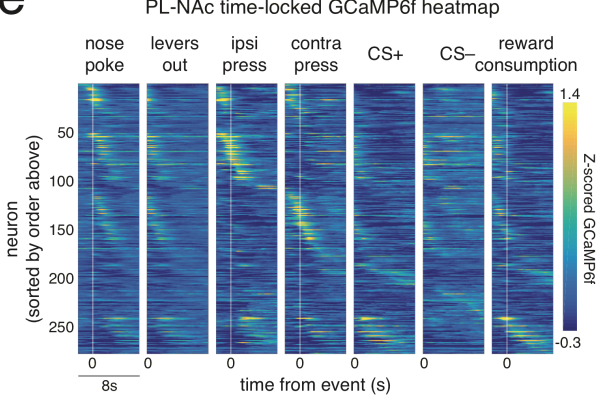

g
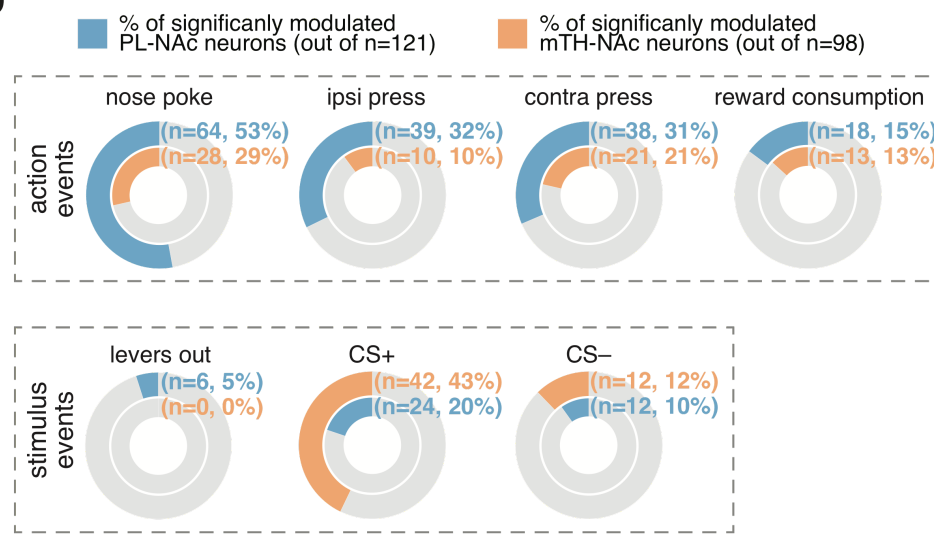

b
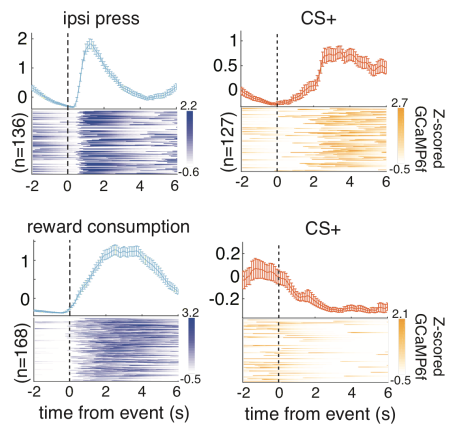

b

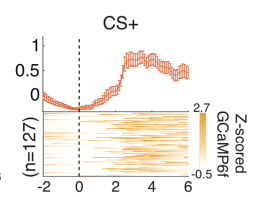

d

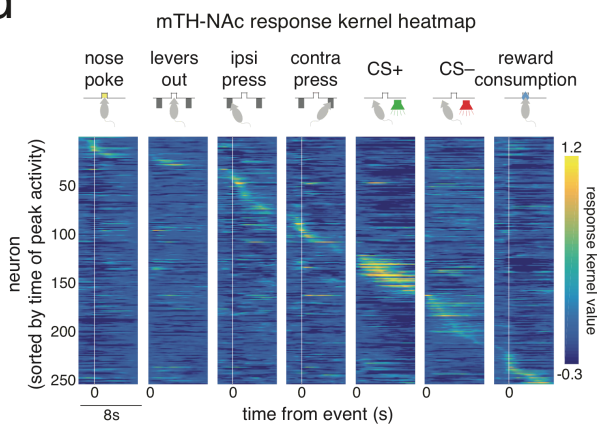

f
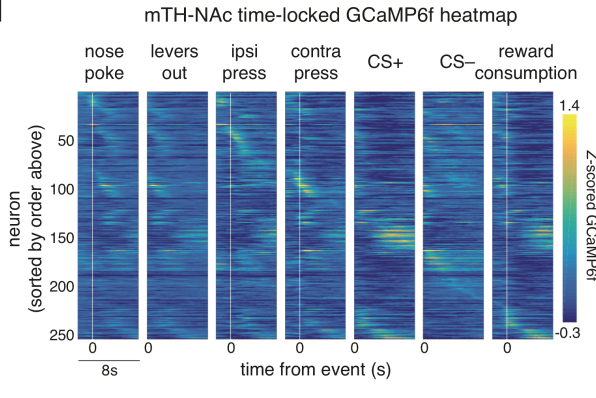

h

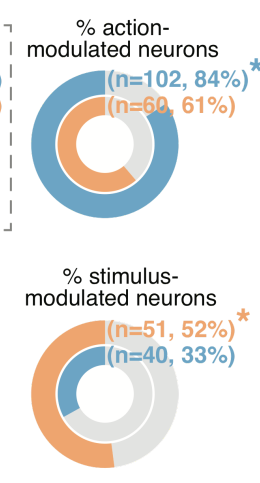

Figure 2 - PL-NAc preferentially represents action events while mTH-NAc preferentially represents the CS+. (a) Time-locked responses of individual PL-NAc (blue) and mTH-NAc (orange) neurons to task events. (b) Kernels representing the response to each of the task events for an example neuron, generated from the encoding model. The predicted GCaMP trace in the model is the sum of the individual response kernels, aligned to the behavioral event times (see Methods). (c) Heatmap of response kernels generated from the encoding model in b from PL-NAc neurons. Heatmap is ordered by the time of the peak response across all behavioral events ( $n=278$ neurons from 7 mice). (d) Same as c except heatmap is of response kernels from mTH-NAc recordings. ( $n=256$ neurons from 9 mice) (e) Heatmap of mean Z-scored GCaMP6f fluorescence from PL-NAc neurons aligned to the time of each event in the task. Neurons are sorted by the order established in c. (f) Same as e except heatmaps are composed of GCaMP6f fluorescence from $\mathrm{mTH}-\mathrm{NAc}$ neuron recordings. (g) Top row, fraction of neurons significantly modulated by action events ('nose poke', 'ipsilateral lever press', 'contralateral lever press', 'reward') in the PL-NAc (blue) and mTH-NAc (orange). For all action events, PL-NAc had a larger fraction of significantly modulated neurons compared with mTH-NAc. Bottom row, fraction of neurons in PL-NAc (blue) and mTH-NAc (orange) significantly modulated by stimulus events ('levers out', 'CS+' and 'CS-'). In contrast to action events, two out of three stimulus events had a larger fraction of significantly modulated neurons in $\mathrm{mTH}-\mathrm{NAc}$ compared with PL-NAc. Significance was determined using the linear model used to generate response kernels in $\mathbf{b}$, see Methods for additional model details. (h) Top, a significantly larger fraction of event-modulated PL-NAc neurons encode at least one action event $(P=0.0001$ : two-proportion Z-test comparing fraction of action-modulated PL-NAc and mTH-NAc neurons). Bottom, same as top except a significantly larger fraction of mTH-NAc neurons encode a stimulus event compared with

PL-NAc ( $\mathrm{P}=0.005$ : two-proportion Z-test comparing fraction of stimulus-modulated neurons between PL-NAc and mTH-NAc). 
bioRxiv preprint doi: https://doi.org/10.1101/725382; this version posted July 17,2020 . The copyright holder for this preprint (which was not certified by peer review) is the author/funder. All rights reserved. No reuse allowed without permission.

a

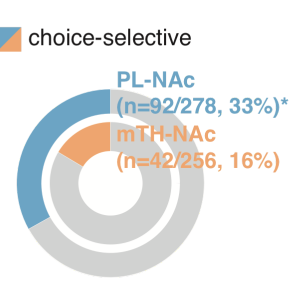

\section{C}

outcome-selective mTH-NAc PL-NAC $(n=70 / 278,25 \%)$

b

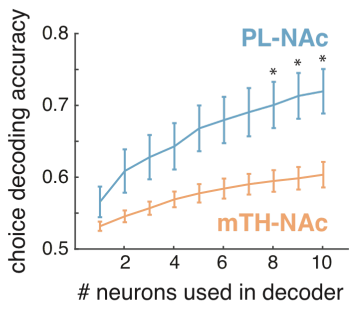

\# neurons used in decoder

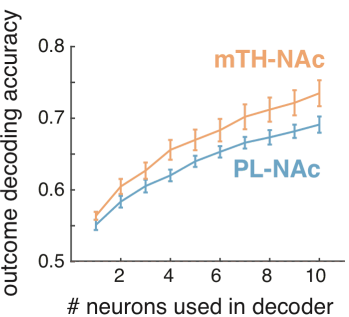

Figure 3 - PL-NAc preferentially represents choice but not outcome relative to mTH-NAc. (a) Fraction of choice-selective neurons in PL-NAc ( $n=92$ out of 278 neurons from 7 mice) and mTH-NAc ( $n=42$ out of 256 neurons from 9 mice). A significantly larger fraction of PL-NAc neurons were choice-selective $\left(P=9.9 \times 10^{-6}\right.$ : two-proportion Z-test comparing fraction of choice-selective PL-NAc and mTH-NAc neurons). (b) Choice decoding accuracy using neural activity from one to ten randomly-selected, simultaneously imaged neurons around the time of the lever press. The PL-NAc population ( $n=6$ mice) more accurately decodes the choice of the trial compared with mTH-NAc ( $n=9$ mice; peak decoding accuracy of $72+/-3 \%$ for PL-NAc and $60+/-2 \%$ for $\mathrm{mTH}-\mathrm{NAc}$ ). Asterisks indicate $\mathrm{P}<0.05$, unpaired two-tailed t-test comparing decoding accuracy between PL-NAc $(n=6)$ and mTH-NAc $(n=9)$ mice. (c) Fraction of outcome-selective neurons in mTH-NAc ( $n=85$ out of 256 neurons, $n=9$ mice) and PL-NAc $(n=70$ out of 278 neurons, $n=7$ mice). A significantly larger fraction of $m T H-N A c$ neurons were outcome-selective $(\mathrm{P}=0.038$ : two-proportion Z-test comparing fraction of outcome-selective PL-NAc and mTH-NAc neurons). (d) Outcome decoding accuracy using neural activity after the time of the CS from one to ten randomly-selected, simultaneously imaged neurons in mTH-NAc in orange $(n=9$ mice, peak decoding accuracy of $74+/-2 \%)$ and PL-NAc in blue $(n=6$ mice, peak decoding accuracy of $69+/-1 \%)$.. Error bars in $\mathbf{b}, \mathbf{d}$ indicate s.e.m. across mice. 
bioRxiv preprint doi: https://doi.org/10.1101/725382; this version posted July 17,2020 . The copyright holder for this preprint (which was not certified by peer review) is the author/funder. All rights reserved. No reuse allowed without permission.

a

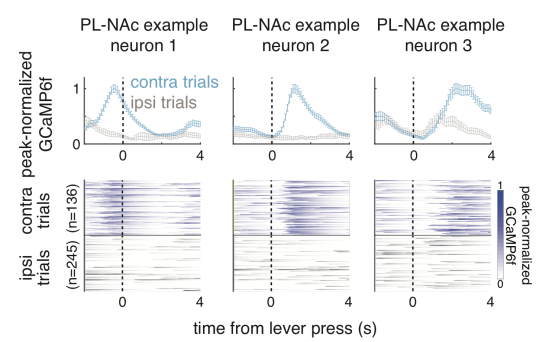

b

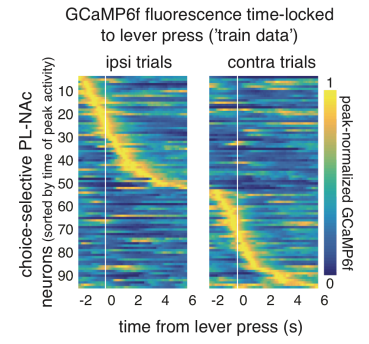

C

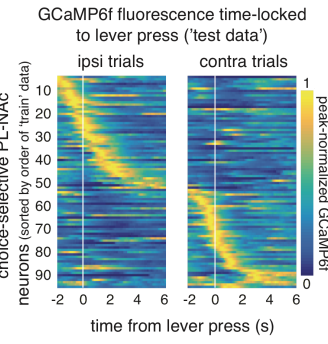

d

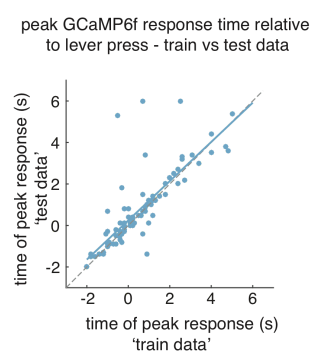

e

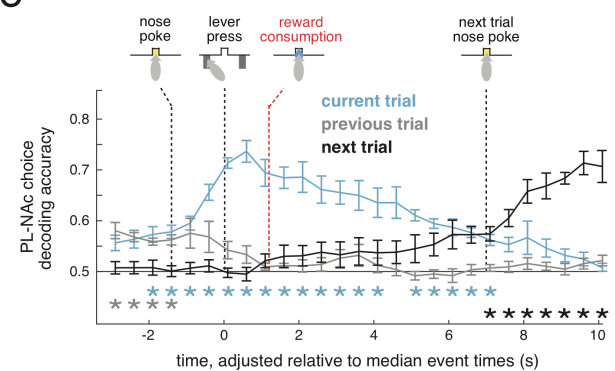

Figure 4 - Choice-selective sequences in PL-NAc persist into the subsequent trial, beyond arrival at the central reward port. (a) Top; average peak-normalized GCaMP6f fluorescence of three simultaneously imaged PL-NAC choice-selective neurons with different response times relative to the lever press. Error bars are s.e.m across trials. Bottom, heatmaps of GCaMP6f fluorescence across trials relative to an ipsilateral (blue) and contralateral press (grey) (b,c) Heatmaps demonstrating sequential activation choice-selective PL-NAc neurons ( $n=92 / 278$ neurons). Each row is the average GCaMP6f fluorescence time-locked to the ipsilateral (left column) and contralateral (right column) lever press for a neuron, normalized by the neuron's peak average

fluorescence. In b ('train data'), heatmap is generated using a randomly selected half of trials and ordered by the time of each neurons' peak activity. In c ('test data'), the peak-normalized, time-locked GCaMP6f fluorescence from the other half of trials was used while maintaining the order from 'train data' in b. This cross-validation procedure ensures that sequences are not an artifact of the sorting. (d) Correlation between time of peak activity using the 'train' (horizontal axis) and 'test' (vertical axis) trials for choice-selective PL-NAc neurons in response to a contralateral or ipsilateral lever press $\left(R^{2}=0.81, P=6.4 \times 10^{-23}, n=92\right.$ neurons). (e) Average decoding accuracy of the mice's choice on the current (blue), previous (grey) and next trial (black) as a function of GCaMP6f fluorescence throughout the current trial. GCaMP6f fluorescence is taken from 100 random selections per mouse of 10 simultaneously imaged PL-NAc neurons (each trial's activity is adjusted in a piecewise linear manner relative to the median time of the nose poke, lever press and next trial nose poke, see Methods for details). Error bars are s.e.m. across mice ( $n=6$ mice). Red dashed line indicates median onset of reward consumption. Asterisks indicate significant decoding accuracy above chance, $\mathrm{P}<0.01$, two-tailed, one-sample t-test across mice. 
a

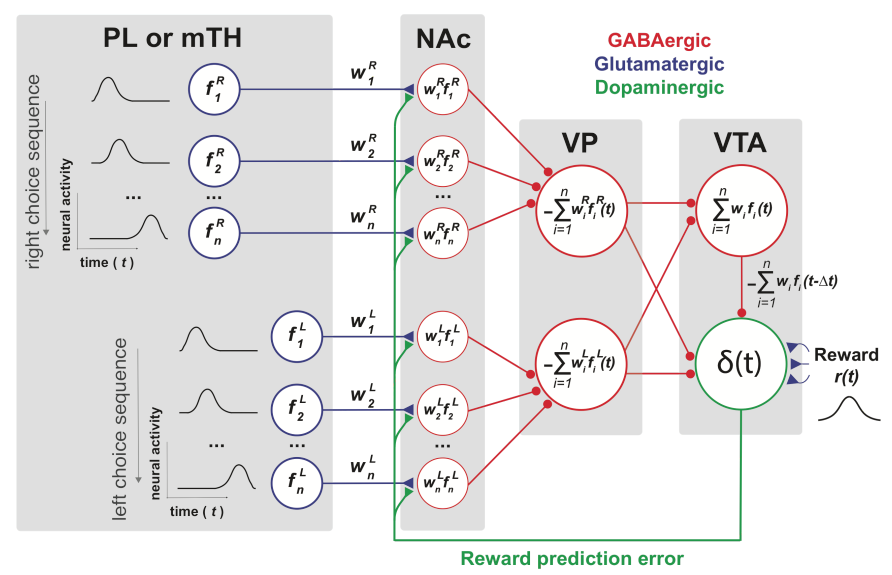

b

$$
\begin{gathered}
V_{L, R}(t)=\sum_{i=1}^{n_{L R}} w_{i}^{L, R}(t) f_{i}^{L, R}(t) \\
V(t)=V_{L}(t)+V_{R}(t) \\
\delta(t)=r(t)+\frac{\gamma V(t)-V(t-\Delta)}{\Delta} \\
\frac{\mathrm{d} w_{i}(t)}{\mathrm{d} t}=\alpha \delta(t) E_{i}(t) \\
\frac{\mathrm{d} E_{i}(t)}{\mathrm{d} t}=\frac{-E_{i}(t)}{\tau_{e}}+f_{i}(t)
\end{gathered}
$$
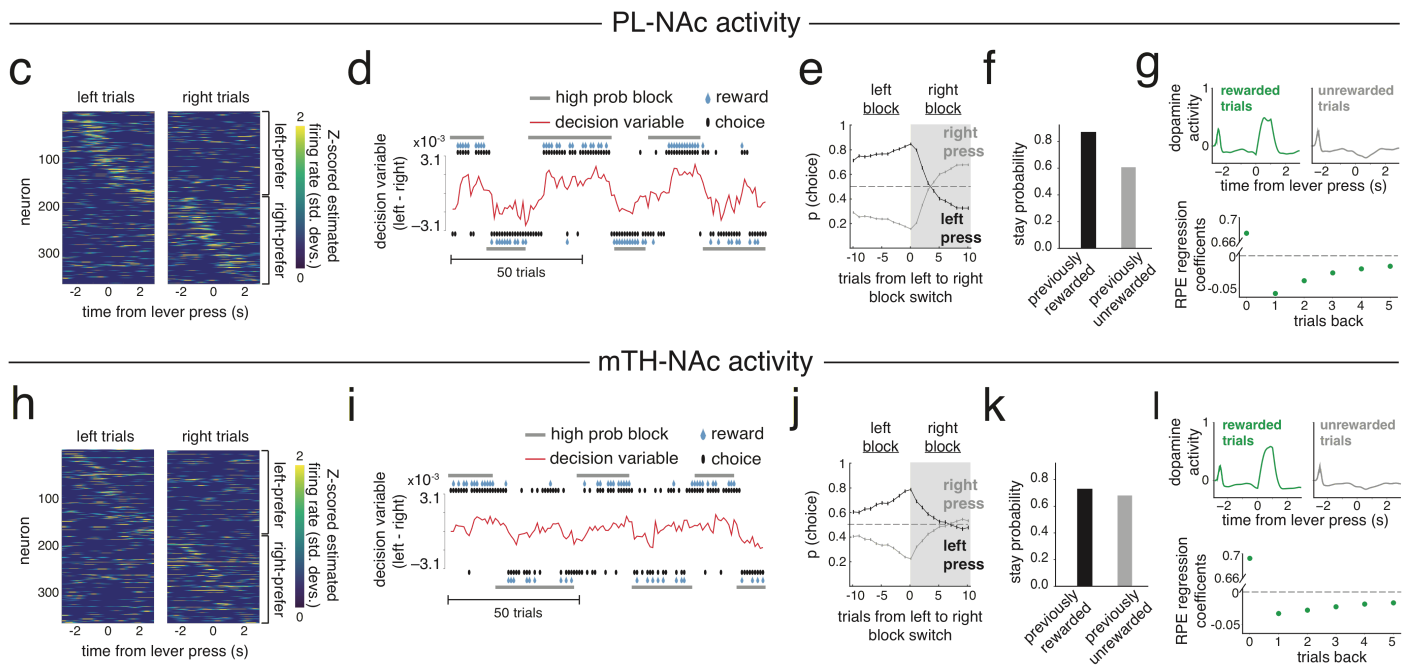

PL-NAc synchronous control

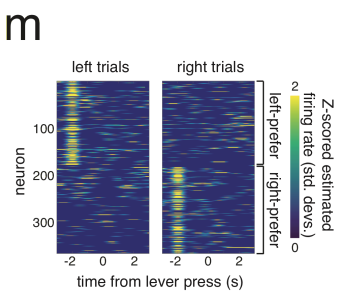

$\mathrm{n}$
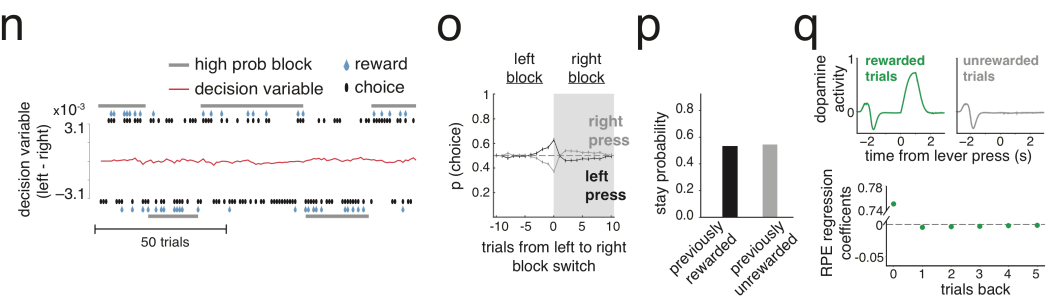

Figure 5 - Choice-selective sequences in PL-NAc, in combination with known downstream connectivity, can implement a temporal difference (TD) learning model. (a) Schematic of circuit architecture used in the model. See Results and Methods for model details. Choice-selective PL-NAc neurons, each with a sequential temporal activity pattern defined as $f_{i}^{R}$ for right-preferring neurons and $f_{i}^{L}$ for left-preferring neurons, form synapses with GABAergic NAc projection neurons with weights $w_{i}^{R}$ and $w_{i}^{L}$. Dopamine (RPE)-dependent changes in the synaptic strengths, $w_{i}^{R, L}$, of these inputs enable the NAc neurons to approximately convey the value of a left or right choice at any time. These NAc neurons converge upon DA neurons through a fast (NAc-VP-DA) and slow, delayed by one synaptic connection (NAc-VP-VTA GABA-DA) pathway to form the temporal differencing operation required to generate the RPE of TD learning. VP neurons serve as a site of convergence (Oorschot 1996; Kimura et al. 1996) and invert the sign of NAc inputs to provide the correct sign for generating an RPE, producing populations representing left or right choice value. DA neurons additionally receive an external reward signal $r(t)$. (b) Equations for the model as instantiated by the circuit schematic in a. $\alpha$ defines the learning rate, $\tau_{e}$ defines the decay time constant for the PL-NAc synaptic eligibility trace $E(t), \Delta$ is the delay generated by the VTA GABA interneuron, $\gamma$ is the discount in value associated with a time step $\Delta$ and $n_{L, R}$ is the total number of left and right-preferring neurons. The individual sums of left and right-preferring NAc neuron activity is given by $V_{L}$ and $V_{R}$, 
bioRxiv preprint doi: https://doi.org/10.1101/725382; this version posted July 17, 2020. The copyright holder for this preprint (which was not certified by peer review) is the author/funder. All rights reserved. No reuse allowed without permission.

respectively, and the sum of the left and right value is $V$. (c) Heatmap of single trial PL-NAc estimated firing rates used as input to the model (see Methods for details). (d) Behavior of the TD model for 120 example trials spanning a series of high-probability block reversals. The decision variable (red trace) along with the predicted choice of the model (black dots) follows the identity of the higher probability lever as it alternates between a left and right block (horizontal grey bars). (e) Choice probability of the model for the left (black) and right (grey) presses following a left-to-right block switch. The model is able to switch to the new lever following a block switch, similar to the mice's behavior in Figure 1c. (f) Predicted stay probability of the TD model following rewarded and unrewarded trials. Similar to the behavior of mice in Figure 1d, the model predicts a higher stay probability following a rewarded trial compared with an unrewarded trial. (g) Top, simulated VTA dopamine neuron activity throughout the trial, averaged across rewarded trials (green) and unrewarded trials (grey). Bottom, coefficients from a linear regression that uses outcome of the current and previous five trials to predict dopamine neuron activity following outcome feedback (see Methods for regression details). Model dopamine activity follows the pattern predicted by the encoding of an RPE as observed in recorded dopamine activity ((Bayer and Glimcher, 2005; Parker et al., 2016); see Supplementary Figure 10 for experimental recordings). (h-I) Same as c-g except using the estimated firing rates from mTH-NAc instead of PL-NAc single trial activity. The mTH-NAc model input generates appropriate behavior; however, the performance is worse than that using PL-NAc input, with less and slower modulation of the decision variables, and weaker modulation of DA activity by previous trial outcomes. $(\mathrm{m})$ Control model designed such that the choice-selective PL-NAc neurons fire synchronously at the approximate onset of the sequence (2s before the lever press), rather than sequentially. ( $\mathbf{n - q}$ ) Same as $\mathbf{d - g}$, except results from using the control model with synchronous choice-selective PL-NAc activity. Unlike the sequential model, synchronous activity is not able to generate appropriate behavior ( $\mathbf{n}-\mathbf{p})$ nor is it able to produce an RPE signal (q). Note that the increase in choice probability just prior to a block switch in the synchronous case (panel $\mathbf{o}$ ), and also in panels $\mathbf{j}$ and $\mathbf{e}$, is due to the statistical structure of the block switching criterion, rather than due to learning (see Methods). 

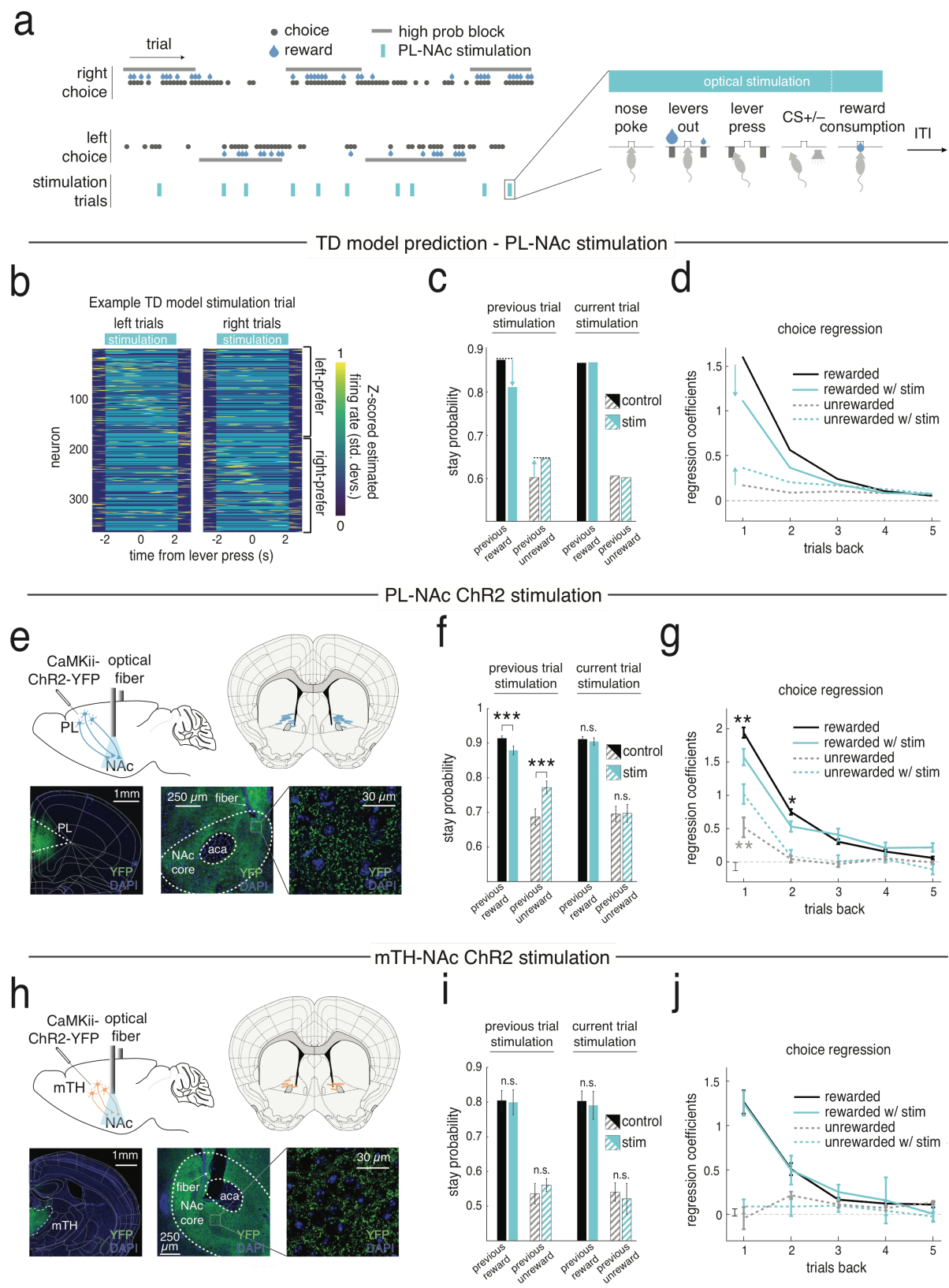

Figure 6 - Stimulation of PL-NAc neurons disrupts the influence of previous trial outcomes on subsequent choice in both the model and in mice. (a) In the TD model and in mice, PL-NAc neurons were stimulated for the full duration of the trial on a randomly selected $10 \%$ of trials, disrupting the endogenous choice-selective sequential activity. For the experimental data, ChR2 stimulation $(447 \mathrm{~nm}, 20 \mathrm{~Hz}$ stimulation, $5 \mathrm{~ms}$ pulse duration, 1-3 $\mathrm{mW}$ ) began when the mouse entered the central nose poke and continued until either $2 s$ after the time of the CS or the end of reward consumption, depending on the outcome of the trial and the experimental cohort (see Methods and Supplementary Figure 13). (b) Heatmap representing single-trial activity of PL-NAc input to the TD model on a stimulation trial. Optogenetic-like stimulation begins at the simulated time of nose poke and ends 2 seconds after CS presentation. (c) Effect of stimulating the PL-NAc input in the TD model. Left, stimulation on the previous trial reduced the stay probability following a stimulation+rewarded trial but increased the stay probability following a stimulation+unrewarded trial. Right, in contrast to the effect of previous trial stimulation, stimulation on the current trial had no effect on the model's stay probability following either rewarded or unrewarded trials. (d) Logistic choice regression showing dependence of the current choice on previous trial choices, outcomes, and presence of stimulation (see Methods). Higher coefficients indicate a higher probability of staying with the previously chosen lever. The model predicts that previous trial stimulation will decrease the stay probability of rewarded trials but increase the stay probability of unrewarded trials across multiple previous trials. (e) Top left, surgical schematic illustrating injection site of an AAV2/5 expressing Camkii-ChR2-eYFP in the PL (black needle) and optical fiber implant in the NAc core terminals. Top right, location of optical fiber tips of PL-NAc ChR2 cohort. Bottom left, coronal section showing ChR2-YFP expression in PL. Bottom middle, ChR2-YFP terminal expression in the NAc-core. Bottom right, ChR2-eYFP expression in PL terminals in the NAc core. (f) Left, as predicted by the model (panel c), PL-NAc ChR2 stimulation on the previous trial significantly reduced the mice's stay probability following a rewarded trial $(P=0.002$ : paired, two-tailed t-test across mice, $n=14)$ while increasing the mice's stay probability following an unrewarded trial $(P=0.0005$ : paired, two-tailed t-test across mice, $n=14)$. Right, also consistent with the model prediction, stimulation on the current trial had no significant effect on stay probability following rewarded $(P=0.62$ : paired, two-tailed t-test across mice, $n=14)$ or unrewarded $(P=0.91$ : paired, two-tailed t-test across mice, $\mathrm{n}=14$ ) trials. (g) Same as $\mathbf{d}$, but for PL-NAc stimulation in mice instead of TD model. Similar to the TD model, PL-NAc ChR2 stimulation decreased the weight of rewarded choices one- and two-trials back $(P=0.002$ : one-trial back; $P=0.023$ : two-trial back: one-sample, two-tailed t-test across mice, $n=14)$ and increased the weight of unrewarded choices one-trial back $\left(P=5.4 \times 10^{-6}\right.$, one-sample, two-tailed t-test across mice, $\left.n=14\right)$. (h-j) Same as e-g but for mTH-NAc ChR2 stimulation. Unlike PL-NAc ChR2 stimulation, mTH-NAc stimulation had no significant effect on the mice's stay probability following either 
bioRxiv preprint doi: https://doi.org/10.1101/725382; this version posted July 17,2020 . The copyright holder for this preprint (which was not certified by peer review) is the author/funder. All rights reserved. No reuse allowed without permission.

rewarded or unrewarded choices on the previous trial (i, P>0.05, paired t-test across mice) and multiple trials back (j, $P>0.05$ for all trials back, t-test across laser $x$ choice interaction term coefficients) 


\section{Methods}

\section{Mice}

46 male C57BL/6J mice from The Jackson Laboratory (strain 000664) were used for these experiments. Prior to surgery, mice were group-housed with 3-5 mice/cage. All mice were $>6$ weeks of age prior to surgery and/or behavioral training. To prevent mice from damaging the implant of cagemates, all mice used in imaging experiments were single housed post-surgery. All mice were kept on a 12-h on/ 12-h off light schedule. All experiments and surgeries were performed during the light off time. All experimental procedures and animal care was performed in accordance with the guidelines set forth by the National Institutes of Health and were approved by the Princeton University Institutional Animal Care and Use Committee.

\section{Probabilistic reversal learning task}

Beginning three days prior to the first day of training, mice were placed on water restriction and given per diem water to maintain $>80 \%$ original body weight throughout training. Mice performed the task in a $21 \times 18 \mathrm{~cm}$ operant behavior box (MED associates, ENV-307W). A shaping protocol of three stages was used to enable training and discourage a bias from forming to the right or left lever. In all stages of training, the start of a trial was indicated by illumination of a central nose poke port. After completing a nose poke, the mouse was presented with both the right and left lever after a temporal delay drawn from a random distribution from 0 to $1 \mathrm{~s}$ in 100 millisecond intervals. The probability of reward of these two levers varied based on the stage of training (see below for details). After the mouse successfully pressed one of the two levers, both retracted and, after a temporal delay drawn from the same uniform distribution, the mice were presented with one of two auditory cues for $500 \mathrm{~ms}$ indicating whether the mouse was rewarded (CS+, $5 \mathrm{kHz}$ pure tone) or not rewarded (CS-, white noise). Concurrent with the CS+ presentation, the mouse was presented with $6 \mu \mathrm{l}$ of $10 \%$ sucrose reward in a dish located equidistantly between the two levers, just interior to the central nose poke. The start time of reward consumption was defined as the moment the mouse first made contact with the central reward port spout following the delivery of the reward. The end of the reward consumption period (ie - reward exit) was defined as the moment in which the mouse was disengaged with the reward port for $>100 \mathrm{~ms}$. In all stages of training, trials were separated by a $2 \mathrm{~s}$ intertrial interval, which began either at the end of CS on unrewarded trials or at the end of reward consumption on rewarded trials.

In the first stage of training (“100-100 debias"), during a two hour session, mice could make a central nose poke and be presented with both the right and left levers, each with a $100 \%$ probability of reward. However, to ensure that mice did not form a bias during this stage, after five successive presses of either lever the mouse was required to press the opposite lever to receive a reward. In this case, a single successful switch to the opposite lever returned both levers to a rewarded state. Once a mouse received $>100$ rewards in a single session they were moved to the second stage ("100-0") where only one of the two levers would result in a reward. The identity of the rewarded lever switched after 10 rewarded trials plus a random number of trials drawn from the geometric distribution:

$$
P(k)=(1-p)^{k-1} p
$$

where $P(k)$ is the probability of a block switch $\mathrm{k}$ trials into a block and $\mathrm{p}$ is the success probability of a switch for each trial, which in our case was 0.4 . After 3 successive days of receiving $>100$ total rewards, the mice were moved to the final stage of training ("70-10"), during which on any given trial 
pressing one lever had a $70 \%$ probability of leading to reward (high-prob lever) while pressing the opposite lever had only a $10 \%$ reward probability (low-prob lever). The identity of the higher probability lever reversed using the same geometric distribution as the 100-0 training stage. On average, there were $23.23+/-7.93$ trials per block and $9.67+/-3.66$ blocks per session (mean +/- std. dev.). In this final stage, the mice were required to press either lever within 10 s of their presentation; otherwise, the trial was considered an 'abandoned trial' and the levers retracted. All experimental data shown was collected while mice performed this final "70-10" stage.

\section{Logistic choice regression}

For the logistic choice regressions shown in Figure 1e and Supplementary Figure 2a, we modeled the choice of the mouse on trial $i$ based on lever choice and reward outcome information from the previous $\mathrm{n}$ trials using the following logistic regression model:

$$
\log \left(\frac{C(i)}{1-C(i)}\right)=\beta_{0}+\sum_{j=1}^{n} \beta_{j}^{R} R(i-j)+\sum_{j=1}^{n} \beta_{j}^{U} U(i-j)+\text { error }
$$

Where $C(i)$ is the probability of choosing the right lever on trial $i, R(i-j)$ and $U(i-j)$ are the choice of the mouse $j$ trials back from the $i$-th trial for either rewarded or unrewarded trials, respectively. $R(i-j)$, was defined as +1 when the $j$-th trial back was both rewarded and a right press, -1 when the $j$-th trial back was rewarded and a left press and 0 when it was unrewarded. Similarly, $U(i-j)$, was defined as +1 when the $j$-th trial back was both unrewarded and a right press, -1 when the $j$-th trial back was unrewarded and a left press and 0 when it was rewarded. The calculated regression coefficients, $\beta_{j}^{R}$ and $\beta_{j}^{U}$, reflect the strength of the relationship between the identity of the chosen lever on a previously rewarded or unrewarded trial, respectively, and the lever chosen on the current trial.

To examine the effect of optogenetic stimulation from multiple trials back on the mouse's choice (Figure 6d,g,j \& Supplementary Figure 13), we expanded our behavioral logistic regression model to include the identity of those trials with optical stimulation, as well as the interaction between rewarded and unrewarded choice predictors and stimulation:

$$
\begin{aligned}
& \log \left(\frac{C(i)}{1-C(i)}\right)=\beta_{0}+\sum_{j=1}^{n} \beta_{j}^{R} R(i-j)+\sum_{j=1}^{n} \beta_{j}^{U} U(i-j)+\ldots \\
& \sum_{j=1}^{n} \beta_{j}^{L R} L(i-j) R(i-j)+\sum_{j=1}^{n} \beta_{j}^{L U} L(i-j) U(i-j)+\sum_{j=1}^{n} \beta_{j}^{L} L(i-j)+\text { error }
\end{aligned}
$$

where $L(i)$ represents optical stimulation on the $i^{\text {th }}$ trial ( 1 for optical stimulation, 0 for control trials), $\beta_{j}^{L}$ represents the coefficient corresponding to the effect of stimulation on choice $\mathrm{j}$ trials back and $\beta_{j}^{L R}$ and $\beta_{j}^{L U}$ represent the coefficients corresponding to the interaction between rewarded choice $\mathrm{x}$ optical stimulation and unrewarded choice $x$ stimulation, respectively. For visualization purposes, in Figure 6d,g,j; Supplementary Figure 13c,d and Supplementary Figure 12d, the solid blue traces represent the sum of the rewarded choice and rewarded choice $x$ stimulation coefficients. Similarly, the dashed blue traces represent the sum of the unrewarded choice and unrewarded choice $x$ 
stimulation coefficients. For all choice regressions, the coefficients for each mouse were fit using the glmfit function in MATLAB and error bars reflect means and s.e.m. across mice.

\section{Cellular-resolution calcium imaging}

To selectively image from neurons which project to the NAc, we utilized a combinatorial virus strategy to image cortical and thalamic neurons which send projections to the NAc. 16 mice (7 PL-NAc, 9 $\mathrm{mTH}-\mathrm{NAc}$ ) previously trained on the probabilistic reversal learning task were unilaterally injected with $500 \mathrm{nl}$ of a retrogradely transporting virus to express Cre-recombinase (CAV2-cre, IGMM vector core, France, injected at $\sim 2.5 \times 10^{12} \mathrm{parts} / \mathrm{ml}$ or retroAAV-EF1a-Cre-WPRE-hGHpA, PNI vector core, injected at $\left.\sim 6.0 \times 10^{13}\right)$ in either the right or left NAc core $(1.2 \mathrm{~mm} \mathrm{~A} / \mathrm{P},+/-1.0 \mathrm{~mm} \mathrm{M} / \mathrm{L},-4.7 \mathrm{D} / \mathrm{V})$ along with $600 \mathrm{nl}$ of a virus to express GCaMP6f in a Cre-dependent manner (AAV2/5-CAG-Flex -GCaMP6f-WPRE-SV40, UPenn vector core, injected at $\sim 1.27 \times 10^{13}$ parts $\left./ \mathrm{ml}\right)$ in either the $\mathrm{mTH}(-0.3$ \& -0.8 A/P, +/- 0.4 M/L, -3.7 D/V) or PL (1.5 \& 2.0 A/P, +/- $0.4 \mathrm{M} / \mathrm{L},-2.5 \mathrm{D} / \mathrm{V})$ of the same hemisphere. 154 of $278(55 \%, n=5$ mice) PL-NAc neurons and 95 out of 256 (37\%, n=5 mice) mTH-NAc neurons were labeled using the CAV2-Cre virus, the remainder were labeled using the retroAAV-Cre virus. In this same surgery, mice were implanted with a $500 \mu \mathrm{m}$ diameter gradient refractive index (GRIN) lens (GLP-0561, Inscopix) in the same region as the GCaMP6f injection - either the PL (1.7 A/P, +/- 0.4 $\mathrm{M} / \mathrm{L},-2.35 \mathrm{D} / \mathrm{V})$ or mMTh (-0.5 A/P, +/- $0.3 \mathrm{M} / \mathrm{L},-3.6 \mathrm{D} / \mathrm{V}) .2-3$ weeks after this initial surgery, mice were implanted with a base plate attached to a miniature, head-mountable, one-photon microscope (nVISTA HD v2, Inscopix) above the top of the implanted lens at a distance which focused the field of view. All coordinates are relative to bregma using Paxinos and Franklin's the Mouse Brain in Stereotaxic Coordinates, 2nd edition (Paxinos and Franklin, 2004). GRIN lens location was imaged using the Nanozoomer S60 Digital Slide Scanner (Hamamatsu) (location of implants shown in Supplementary Figure 1). The subsequent image of the coronal section determined to be the center of the lens implant was then aligned to the Allen Brain Atlas (Allen Institute, brain-map.org) using the Wholebrain software package (wholebrainsoftware.org, (Fürth et al., 2018)).

Post-surgery, mice with visible calcium transients were then retrained on the task while habituating to carrying a dummy microscope attached to the implanted baseplate. After the mice acclimated to the dummy microscope, they performed the task while images of the recording field of view were acquired at $10 \mathrm{~Hz}$ using the Mosaic acquisition software (Inscopix). To synchronize imaging data with behavioral events, pulses from the microscope and behavioral acquisition software were recorded using either a data acquisition card (USB-201, Measurement computing) or, when LED tracking (see below for details) was performed, an RZ5D BioAmp processor from Tucker-Davis Technologies. Acquired videos were then pre-processed using the Mosaic software and spatially downsampled by a factor of 4. Subsequent down-sampled videos then went through two rounds of motion-correction. First, rigid motion in the video was corrected using the translational motion correction algorithm based on Thévenaz et al. (1998) included in the Mosaic software (Inscopix, motion correction parameters: translation only, reference image: the mean image, speed/accuracy balance: 0.1 , subtract spatial mean [ $r=20$ pixels], invert, and apply spatial mean $[r=5$ pixels]). The video then went through multiple rounds of non-rigid motion correction using the NormCore motion correction algorithm (Pnevmatikakis and Giovannucci, 2017) NormCore parameters: gSig=7, gSiz=17, grid size and grid overlap ranged from 12-36 and 8-16 pixels, respectively, based on the individual motion of each video. Videos underwent multiple (no greater than 3 ) iterations of NormCore until non-rigid motion was no longer visible). Following motion correction, the CNMFe algorithm (Zhou et al., 2018) was used to extract the fluorescence traces (referred to as 'GCaMP6f' throughout the text) as well as an estimated firing rate of each neuron (CNMFe parameters: spatial downsample factor $=1$, temporal downsample=1, gaussian kernel width $=4$, maximum neuron diameter $=20$, tau decay=1, tau rise=0.1). Only those neurons with an estimated firing rate of four transients/ minute or higher were considered 
'task-active' and included in this paper - 278/330 (84\%; each mouse contributed 49,57,67,12,6,27,60 neurons, respectively) of neurons recorded from PL-NAc passed this threshold while 256/328 (78\%; each mouse contributed 17,28,20,46,47,40,13,13,32 neurons, respectively) passed in mTH-NAc. Across all figures, to normalize the neural activity across different neurons and between mice, we Z-scored each GCaMP6f recording trace using the mean and standard deviation calculated using the entire recording session.

\section{Encoding model to generate response kernels for behavioral events}

To determine the response of each neuron attributable to each of the events in our task, we used a multiple linear encoding model with lasso regularization to generate a response kernel for each behavioral event (example kernels shown in Figure $\mathbf{2 b}$ ). In this model, the dependent variable was the GCaMP6f trace of each neuron recorded during a behavioral session and the independent variables were the times of each behavioral event ('nose poke', 'levers out', 'ipsilateral lever press', 'contralateral lever press', 'CS+', 'CS-' and 'reward consumption) convolved with a 25 degrees-of-freedom spline basis set that spanned -2 to $6 s$ before and after the time of action events ('nose poke', ipsilateral press', 'contralateral press' and 'reward consumption) and 0 to $8 \mathrm{~s}$ from stimulus events ('levers out', 'CS+' and 'CS-'). To generate this kernel, we used the following linear regression with lasso regularization using the lasso function in MATLAB:

$$
\min _{\beta_{0}, \beta_{j k}}\left(\sum_{t=1}^{T}\left(F(t)-\sum_{k=1}^{K} \sum_{j=1}^{N_{s p}} \beta_{j k} X_{j k}(t)-\beta_{0}\right)^{2}+\lambda \sum_{k=1}^{K} \sum_{j=1}^{N_{s p}}\left|\beta_{j k}\right|\right)
$$

where $F(t)$ is the Z-scored GCaMP6f fluorescence of a given neuron at time $t, T$ is the total time of recording, $K$ is the total number of behavioral events used in the model, $N_{s p}$ is the degrees-of-freedom for the spline basis set (25 in all cases), $\beta_{j k}$ is the regression coefficient for the $j^{\text {th }}$ spline basis function and $k^{\text {th }}$ behavioral event, $\beta_{0}$ is the intercept term and $\lambda$ is the lasso penalty coefficient. The value of lambda which minimized the mean squared error of the model, as determined by 5 -fold cross validation, was used. The predictors in our model, $X_{j k}$ were generated by convolving the behavioral events with a spline basis set, to enable temporally delayed versions of the events to predict neural activity:

$$
X_{j k}(t)=\sum_{i=1}^{N=81} S_{j}(i) e_{k}(t-i)
$$

where $S_{j}(i)$ is the $j^{t h}$ spline basis function at time point $i$ with a length of 81 time bins (time window of -2 to $6 \mathrm{~s}$ for action events or 0 to $8 \mathrm{~s}$ for stimulus events sampled at $10 \mathrm{~Hz}$ ) and $e_{k}$ is a binary vector of length $T$ representing the time of each behavioral event $k$ ( 1 at each time point where a behavioral event was recorded using the MED associates and TDT software, 0 at all other timepoints).

Using the regression coefficients, $\beta_{j k}$, generated from the above model, we then calculated a 'response kernel' for each behavioral event: 


$$
\text { kernel }_{k}(t)=\sum_{j=1}^{N_{s p}} \beta_{j k} S_{j}(t)
$$

This kernel represents the (linear) response of a neuron to each behavioral event, while accounting for the linear component of the response of this neuron to the other events in the task.

\section{Quantification of neural modulation to behavioral events}

To identify neurons that were significantly modulated by each of the behavioral events in our task (fractions shown in Figure $\mathbf{2 g}, \mathbf{h}$ ), we used the encoding model described above, but without the lasso regularization:

$$
F(t)=\beta_{0}+\sum_{k=1}^{K} \sum_{j=1}^{N_{s p}} \beta_{j k} X_{j k}(t)
$$

As above, $F(t)$ is the Z-scored GCaMP6f fluorescence of a given neuron at time $t, K$ is the total number of behavioral events used in the model, $N_{s p}$ is the degrees-of-freedom for the spline basis set (25 in all cases), $\beta_{j k}$ is the regression coefficient for the $j^{\text {th }}$ spline basis function and $k^{\text {th }}$ behavioral event and $\beta_{0}$ is the intercept term. To determine the relative contribution of each behavioral event when predicting the activity of a neuron, we compared the full version of this model to a reduced model with the $X$ and $\beta$ terms associated with the behavioral event in question excluded. For each behavioral event, we first generated an F-statistic by comparing the fit of a full model containing all event predictors with that of a reduced model that lacks the predictors associated with the event in question. We then calculated this same statistic on 500 instances of shuffled data, where shuffling was performed by circularly shifting the GCaMP6f fluorescence by a random integer. We then compared the F-statistic from the real data to the shuffled distribution to determine whether the removal of an event as a predictor compromised the model significantly more than expected by chance. If the resulting $P$-value was less than the significance threshold of $P=0.01$, after accounting for multiple comparison testing of each of the behavioral events by Bonferroni correction, then the event was considered significantly encoded by that neuron.

To determine whether a neuron was significantly selective to the choice or outcome of a trial ('choice-selective' and 'outcome-selective', fractions of neurons from each population shown in Figure 3a,c), we utilized a nested model comparison test similar to that used to determine significant modulation of behavioral events above, where the full model used the following behavioral events as predictors: 'nose poke', levers out', 'all lever press', 'ipsilateral lever press', 'all CS', 'CS+' and 'reward consumption'. For choice-selectivity, an F-statistic was computed for a reduced model lacking the 'ipsilateral lever press' predictors and significance was determined by comparing this value with a null distribution generated using shuffled data as described above. For outcome-selectivity, the reduced model used to test for significance lacked the predictors associated with both the 'CS+' and 'reward consumption' events.

By separating the lever press and outcome-related events into predictors that were either blind to the choice or outcome of the trial ('all lever press' and 'all CS', respectively) and those which included choice or outcome information ('ipsilateral lever press' or 'CS+' and 'reward consumption', respectively) we were able to determine whether the model was significantly impacted by the removal of either choice or outcome information. Therefore, neurons with significant encoding of the 'ipsilateral 
lever press' event (using the same P-value threshold determined by the shuffled distribution of F-statistics) were considered choice-selective, while those with significant encoding of the 'CS+/reward consumption' events were considered outcome-selective.

\section{Neural decoders}

Choice decoder In Figure 3b, we quantified how well simultaneously-imaged populations of 1 to 10 PL-NAc or mTH-NAc neurons could be used to decode choice using a logistic regression:

$$
\log \left(\frac{C(i)}{1-C(i)}\right)=\beta_{0}+\sum_{j=1}^{n} \beta_{j} X_{j}(i)
$$

where $\mathrm{C}(\mathrm{i})$ is the probability the mouse made an ipsilateral choice on trial $\mathrm{i}, \beta_{0}$ is the offset term, $\mathrm{n}$ is the number of neurons (between 1 and 10), $\beta_{j}$ is the regression weight for each neuron, and $X_{j}$ is the mean z-scored GCaMP6f fluorescence from -2 to 6 seconds around the lever press on trial $i$.

Given that the mice's choices were correlated across neighboring trials, we weighted the logistic regression based on the frequency of each trial type combination. This was to ensure that choice decoding of a given trial was a reflection of the identity of the lever press on the current trial as opposed to that of the previous or future trial. Thus, we classified each trial as one of eight 'press sequence types' based on the following 'previous-current-future' press sequences: ipsi-ipsi-ipsi, ipsi-ipsi-contra, ipsi-contra-contra, ipsi-contra-ipsi, contra-contra-contra, contra-contra-ipsi, contra-ipsi-ipsi, contra-ipsi-contra. We then used this classification to equalize the effects of press-sequence type on our decoder by multiplying each predictor (i.e. the average neural activity of a trial) by a weight corresponding to the inverse of the frequency of the press sequence type of that trial.

The logistic regression was fit using the fitglm function in MATLAB. Decoder performance was evaluated with 5 -fold cross-validation by calculating the proportion of correctly classified held-out trials. Predicted ipsilateral press probabilities greater than or equal to 0.5 were decoded as an ipsilateral choice and values less than 0.5 were decoded as a contralateral choice. This was repeated with 100 combinations of randomly-selected, simultaneously-imaged neurons from each mouse. Reported decoding accuracy is the average accuracy across the 100 runs and 5 combinations of train-test data for each animal. Note that only 6/7 mice in the PL-NAc cohort were used in the decoder analyses as one mouse had fewer than 10 simultaneously imaged neurons.

Outcome decoder For the outcome decoder in Figure 3d, we used the same weighted logistic regression used for choice decoding, except the dependent variable was the outcome of the trial $(+1$ for a reward, 0 for no reward) and the predictors were the average GCaMP6f fluorescence during the intertrial interval (ITI) of each trial. The ITI was defined as the time between CS presentation and either 1s before the next trial's nose poke or 8s after the CS, whichever occurred first. This was used in order to avoid including any neural activity attributable to the next trial's nose poke in our analysis.

To correct for outcome correlations between neighboring trials, we performed a similar weighting of predictors as performed in the choice decoder above using the following eight outcome sequence 
types: 'reward- reward- reward', 'reward- reward- unreward', 'reward- unreward- unreward', 'rewardunreward- reward', 'unreward- unreward- unreward', 'unreward- unreward- reward', 'unrewardreward- reward', 'unreward- reward- unreward.'

Time-course choice decoder To determine how well activity from PL-NAc and mTH-NAc neurons was able to predict the mouse's choice as a function of time throughout the trial (Figure $4 \mathbf{e} \&$ Supplementary Figure 7e), we trained separate logistic regressions on 500 millisecond bins throughout the trial, using the GCaMP6f fluorescence of 10 simultaneously imaged neurons.

Because of the variability in task timing imposed by the jitter and variability of the mice's actions, we linearly interpolated the GCaMP6f fluorescence trace of each trial to a uniform length, $t_{\text {adjusted }}$, relative to behavioral events in our task. Specifically, for each trial, $T$, we divided time into the following four epochs: (i) 2 seconds before nose poke, (ii) time from the nose poke to the lever press, (iii) time from the lever press to the nose poke of the subsequent trial, $T+1$ and (iv) the 3 seconds following the next trial nosepoke. For epochs $\mathrm{ii}$ and iii, $t_{\text {adjusted }}$ was determined by interpolating the GCaMP6f fluorescence trace from each trial to a uniform length defined as the median time between the flanking events across all trials. Thus, $t_{\text {adjusted }}$ within each epoch for each trial, $T$, was defined as:

$$
T_{\text {adjusted }}(t) \equiv \begin{cases}t & , t_{n p}^{T}-2 \leq t<t_{n p}^{T} \\ 2+\frac{\left(t-t_{n p}^{T}\right)}{\left(t_{l p}^{T}-t_{n p}^{T}\right)} \widetilde{e p_{i i}} & , t_{n p}^{T} \leq t<t_{l p}^{T} \\ 2+\widetilde{e p_{i i}}+\frac{\left(t-t_{l p}^{T}\right)}{\left(t_{n p}^{T+1}-t_{l p}^{T}\right)} \widetilde{e p_{i i i}} & , t_{l p}^{T} \leq t<t_{n p}^{T+1} \\ t & , t_{n p}^{T+1} \leq t<t_{n p}^{T+1}+3\end{cases}
$$

where $t_{n p}^{T}$, and $t_{l p}^{T}$ are the times of the nose poke and lever press on the current trial, $t_{n p}^{T+1}$ is the time of the nose poke of the subsequent trial and $\widetilde{e p_{i i}}$ and $\widetilde{e p_{i i i}}$ are the median times across trials of epoch ii and iii.

The resulting time-adjusted GCaMP6f traces were divided into 500 millisecond bins. For each bin, we fit the weighted logistic regression described above to predict choice on the current, previous or future trial from the activity of 10 simultaneously-imaged neurons. Predictors were weighted based on press sequence type as described above. Decoding accuracy was assessed as described above using 100 combinations of 10 randomly-selected neurons and 5-fold cross-validation (Figure 4e). To determine if decoding was significantly above chance, which is 0.5 , for each timepoint we performed a two-tailed, one-sample t-test.

\section{Statistics}

All t-tests reported in the results were performed using either the ttest or ttest2 function in Matlab. In all cases, t-tests were two-tailed. In cases where multiple comparisons were performed, we applied a Bonferroni correction to determine the significance threshold. Two-proportion Z-tests (used to compare fractions of significantly modulated/selective neurons, Figures $\mathbf{2 g}, \mathbf{h} \& \mathbf{3 a}, \mathbf{c}$ ) and Fisher's $Z$ (used to compare correlation coefficients, Figure 4d \& Supplementary Figure 7d) were performed using Vassarstats.net. 
For all $t$-tests in this paper, data distributions were assumed to be normal, but this was not formally tested. No statistical methods were used to predetermine sample sizes, but our sample sizes were similar to those generally employed in the field.

\section{TD-learning model: Theory}

To computationally model how the brain could solve the reversal learning task, we generated a biological instantiation of the TD algorithm for reinforcement learning (Sutton and Barto, 1998) by combining the recorded PL-NAc activity with known circuit connectivity in the NAc and associated regions (Hunnicutt et al., 2016; Kalivas et al., 1993; Otis et al., 2017; Watabe-Uchida et al., 2012). The goal of the model is to learn the value of each choice at the onset of the PL-NAc sequence, so it can be used to drive the choice, despite the fact that the reward occurs later in the sequence.

The Value Function Our implementation of the TD algorithm seeks to learn an estimate, at any given time, of the total discounted sum of expected future rewards, known as the value function $V(t)$. To do this, we assume that the value function over time is decomposed into a weighted sum of temporal basis functions (Sutton and Barto, 1998) corresponding to the right-choice and left-choice preferring PL-NAc neurons:

$$
\begin{aligned}
& V_{R}(t)=\sum_{i=1}^{n_{R}} w_{i}^{R}(t) f_{i}^{R}(t) \\
& V_{L}(t)=\sum_{i=1}^{n_{L}} w_{i}^{L}(t) f_{i}^{L}(t)
\end{aligned}
$$

with the total value being given by the sum over both the left and right neurons as

$$
V(t)=V_{R}(t)+V_{L}(t)
$$

Here, $V_{R}(t)$ and $V_{L}(t)$ are the components of the value functions encoded by the right- and left-preferring neurons respectively, $n_{R}$ and $n_{L}$ are the number of right- and left-preferring choice-selective neurons respectively, and $w_{i}^{R, L}$ are the weights between the $i^{\text {th }} \mathrm{PL}$ neuron and the NAc, which multiply the corresponding basis functions. Thus, each term in $V_{R}(t)$ or $V_{L}(t)$ above corresponds to the activity of one of the striatal neurons in the model (Figure 5a). Note that in our model the total value $V(t)$ sums the values associated with the left and right actions and is thus not associated with a particular action. At any given time on a given trial, however, only one or the other choice-selective sequence is active (see Figure 5c), so that a single sequence, corresponding to the chosen action, gets reinforced.

The prediction error. TD learning updates the value function iteratively by computing errors in the predicted value function and using these to update the weights $w_{i}$. The prediction error at each moment of time is calculated from the change in the estimated value function over a time step of size $d t$ as follows

$$
\text { Prediction Error }=\delta(t) \mathrm{d} t=r(t) \mathrm{d} t+e^{\frac{-\mathrm{d} t}{\tau}} V(t)-V(t-\mathrm{d} t)
$$


where $\delta(t)$ is the prediction error per unit time. Here, the first two terms represent the estimated value at time $t$, which equals the sum of the total reward received at time $t$ and the (discounted) expectation of rewards, i.e. value, at all times into the future. This is compared to the previous time step's estimated value $V(t-d t)$. The coefficient $e^{\frac{-\mathrm{d} t}{\tau}}$ represents the temporal discounting of rewards incurred over the time step $\mathrm{d} t$. Here $\tau$ denotes the timescale of temporal discounting and was chosen to be $0.8 s$.

To translate this continuous time representation of prediction error signals to our biological circuit model, we assume that the prediction error $\delta(t)$ is carried by dopamine neurons (Montague et al., 1996; Schultz et al., 1997). These dopamine neurons receive three inputs corresponding to the three terms on the right side of the above equation: a reward signal originating from outside the VTA, a discounted estimate of the value function $V(t)$ from the striatum via the ventral pallidum (Chen et al., 2019; Tian et al., 2016) and an oppositely signed, delayed copy of the value function $V(t-\Delta)$ that converges upon the VTA interneurons (Cohen et al., 2012).

Because the analytical formulation of TD learning in continuous time is defined in terms of the infinitesimal time step $d t$, but a realistic circuit implementation needs to be characterized by a finite delay time for the disynaptic pathway through the VTA interneurons, we rewrite the above equation approximately for small, but finite delay $\Delta$ as:

$$
\delta(t) \mathrm{d} t=r(t) \mathrm{d} t+\frac{\gamma V(t)-V(t-\Delta)}{\Delta} \mathrm{d} t
$$

where we have defined $\gamma=e^{\frac{-\Delta}{\tau}}$ as the discount factor corresponding to one interneuron time delay and, in all simulations, we chose a delay time $\Delta=0.01 \mathrm{~s}$. Note that the discount factor is biologically implemented in different strengths of the weights in the VP input to the GABA interneuron and dopaminergic neuron in the VTA.

The proposed circuit architecture of Figure 5a can be rearranged into several other, mathematically equivalent architectures (Supplementary Figure 9). These architectures are not mutually exclusive, so other more complicated architectures could be generated by superpositions of these architectures.

The eligibility trace. The prediction error at each time step $\delta(t) \mathrm{d} t$ was used to update the weights of the recently activated synapses, where the "eligibility" $E_{i}(t)$ of a synapse for updating depends upon an exponentially weighted average of its recent past activity (Gerstner et al., 2018; Sutton and Barto, 1998):

$$
E_{i}(t)=\int_{-\infty}^{t} e^{\frac{s-t}{\tau_{e}}} f_{i}(s) \mathrm{d} s
$$

which can be rewritten as

$$
\frac{\mathrm{d} E_{i}(t)}{\mathrm{d} t}=-\frac{E_{i}(t)}{\tau_{e}}+f_{i}(t)
$$

or, in the limit of $d t<<1$, 


$$
E_{i}(t) \approx e^{-\frac{\mathrm{d} t}{\tau_{e}}} E_{i}(t-\mathrm{d} t)+f_{i}(t) \mathrm{d} t
$$

where $\tau_{e}$ defines the time constant of the decay of the eligibility trace, which was chosen to be $0.6 \mathrm{~s}$ consistent with (Gerstner et al., 2018; Yagishita et al., 2014).

Weight Updates. The weight of each PL-NAc synapse, $w_{i}$, is updated according to the product of its eligibility $E_{i}(t)$ and the prediction error rate $\delta(t)$ at that time using the following update rule (Gerstner et al., 2018; Sutton and Barto, 1998) :

$$
\begin{gathered}
\frac{\mathrm{d} \hat{w}_{i}(t)}{\mathrm{d} t}=\alpha \delta(t) E_{i}(t) \\
w_{i}(t)=\max \left[0, \hat{w}_{i}(t)\right]
\end{gathered}
$$

where $\alpha=0.009(\text { spikes } / s)^{-1}$ was the learning rate. Note that the units of $\alpha$ derive from the units of weight being value $\cdot(\text { spikes/s) })^{-1}$. The PL-NAc weights used in the model are thresholded to be non-negative so that the weights obey Dale's principle.

Action Selection. In the model, the decision to go left or right is determined by "probing" the relative values of the left versus right actions just prior to the start of the choice-selective sequence. To implement this, we assumed that the choice was read out in a noisy, probabilistic manner from the activity of the first 60 neurons in each (left or right) PL population prior to the start of the sequential activity. This was accomplished by providing a $50 \mathrm{~ms}$ long, noisy probe input to each of these PL neurons and reading out the summed activity of the left and the summed activity of the right striatal populations. The difference between these summed activities was then put through a softmax function (given below) to produce the probabilistic decision.

To describe this decision process quantitatively, we define the probability of making a leftward or rightward choice in terms of underlying decision variables $d_{\text {left }}$ and $d_{\text {right }}$ corresponding to the summed activity of the first 60 striatal neurons in each population:

$$
\begin{aligned}
d_{\text {left }} & =\mathbb{E}_{t}\left[\sum_{i=1}^{60} w_{i}^{\text {left }} n_{i}^{\text {left }}(t)\right] \\
d_{\text {right }} & =\mathbb{E}_{t}\left[\sum_{i=1}^{60} w_{i}^{\text {right }} n_{i}^{\text {right }}(t)\right]
\end{aligned}
$$

where $\mathbb{E}_{t}[$.$] denotes time-averaging over the 50 \mathrm{~ms}$ probe period and $n_{i}^{\text {left }}(t)$ and $n_{i}^{\text {right }}(t)$ denote the non-negative stochastic probe input, which was chosen independently for each neuron and each time step from a normal distribution with mean equal to $0.05 \mathrm{~s}^{-1}$ ( $5 \%$ of peak activity) and a standard deviation of $0.0025 / \sqrt{\mathrm{d} t} \mathrm{~s}^{-1}$. Note that the weights $w_{i}^{\text {left/right }}$ used here correspond to the weights from the end of the previous trial, which we assume are the same as the weights at the beginning of 
the next trial. The probability of choosing the left or the right lever for a given trial $n$ is modeled as a softmax function of these decision variables plus a "stay with the previous choice" term that models the tendency of mice in our study to return to the previously chosen lever irrespective of reward (Figure 1d), given by the softmax distribution

$$
\begin{aligned}
\operatorname{Prob}(\text { left }) & =\frac{\exp \left(\beta_{\text {value }} d_{\text {left }}+\beta_{\text {stay }} I_{\text {left }}\right)}{\exp \left(\beta_{\text {value }} d_{\text {left }}+\beta_{\text {stay }} I_{\text {left }}\right)+\exp \left(\beta_{\text {value }} d_{\text {right }}+\beta_{\text {stay }} I_{\text {right }}\right)} \\
\operatorname{Prob}(\text { right }) & =\frac{\exp \left(\beta_{\text {value }} d_{\text {right }}+\beta_{\text {stay }} I_{\text {right }}\right)}{\exp \left(\beta_{\text {value }} d_{\text {left }}+\beta_{\text {stay }} I_{\text {left }}\right)+\exp \left(\beta_{\text {value }} d_{\text {right }}+\beta_{\text {stay }} I_{\text {right }}\right)}
\end{aligned}
$$

where $I_{l e f t / r i g h t}$ is 1 if that action (i.e. left or right) was chosen on the previous trial and 0 otherwise and $\beta_{\text {value }}=2500$ and $\beta_{\text {stay }}=0.2$ are free parameters that define the width of the softmax distribution and the relative weighting of the value-driven versus stay contributions to the choice.

\section{Model implementation}

Block structure for the model. Block transitions were determined using the same criteria as in the probabilistic reversal learning task performed by the mice - the identity of the rewarded lever switched after 10 rewarded trials plus a random number of trials drawn from the geometric distribution given by Equation 1. In our case, $p=0.4$ was used in both the model and the reversal learning task. Given the variation in performance across the models that use PL-NAc, mTH-NAc or PL-NAc synchronous activity as input (see Figure 5), the average block length for each model varied as well (as block transitions depended upon the number of rewarded trials). The average block length for the single-trial PL-NAc model, single-trial mTH-NAc model and PL-NAc synchronous control were 22.6+/-6.3, 25.7+/-8.5 and 26.5+/-6.6 trials (mean+/-std. dev.), respectively. The PL-NAc model produced a similar block length as that of behaving mice $(23.23+/-7.93$ trials, mean+/- std. dev.). Because a block switch in our task is dependent on the mice receiving a set number of rewards, the choices just prior to a block switch are more likely to align with the identity of the block and result in reward (see

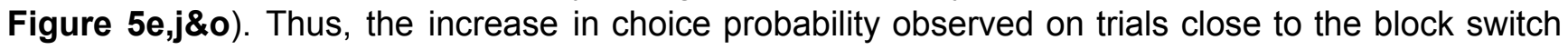
(most notably in the synchronous case in Figure 5o, but also notable in the mTH-NAc in Figure 5j and the PL-NAc in Figure 5e) is an artifact of this switching rule and not reflective of the model learning choice values.

PL-NAc inputs to the neural circuit model. To generate the temporal basis functions, $f_{i}(t)$ (Figure 5c), we used the choice-selective sequential activity recorded from the PL-NAc neurons shown in Figure 4b. Spiking activity was inferred from calcium fluorescence using the CNMFe algorithm (Zhou et al., 2018), and choice-selectivity was determined using the nested comparison model described in Methods above and used to generate Figure 3a. Model firing rates were generated by Z-scoring the inferred spiking activity of each choice-selective PL-NAc neuron.

To generate a large population of model input neurons on each trial, we created a population of 368 choice-selective "pseudoneurons" on each trial. This was done as follows: for each simulated trial, we created 4 copies (pseudoneurons) each of the 92 recorded choice-selective PL-NAc neurons using 
that neuron's inferred spiking activity from 4 different randomly selected trials. The pool of experimentally recorded trials from which pseudoneuron activities were chosen was balanced to have equal numbers of stay and switch trials. This was done because the choices of the mice were strongly positively correlated from trial to trial (i.e., had more stay than switch trials), which (if left uncorrected) potentially could lead to biases in model performance if activity late in a trial was reflective of choice on the next, rather than the present trial. To avoid choice bias in the model, we combined the activity of left and right-choice-preferring recorded neurons when creating the pool of pseudoneurons. We then randomly selected 184 left-choice-preferring and 184 right-choice-preferring model neurons from this pool of pseudoneurons. An identical procedure, using the 92 most choice-selective mTH-NAc neurons, was followed to create the model mTH-NAc neurons. The identity of these 92 neurons was determined by ranking each neurons' choice-selectivity using the p-value calculated to determine choice-selectivity (see Quantification of neural modulation to behavioral events above for details).

To generate the synchronous PL-NAc activity used in Figure $5 \mathbf{h}-\mathbf{I}$, the temporal basis function of each $\mathrm{PL}$ neuron was time-shifted so the peak probability of firing was $2 \mathrm{~s}$ before the time of the lever press.

To mimic the PL-NAc activity during the optogenetic stimulation of PL-NAc neurons (Figure 6b), we set $f_{i}^{R, L}(t)$ equal to 0.3 for a randomly selected $65 \%$ of PL neurons, at all times $t$, from the time of the simulated nosepoke to two seconds after the reward presentation. These 'stimulation trials' occurred on a random $10 \%$ of trials. $65 \%$ of $\mathrm{PL}$ neurons were activated to mimic the incomplete penetrance of ChR2 viral expression.

Reward input to the neural circuit model. The reward input $r(t)$ to the dopamine neurons was modeled by a Gaussian temporal profile centered at the time of the peak reward:

$$
r(t)=R(i) \frac{1}{\sqrt{2 \pi \sigma_{r}^{2}}} e^{-\frac{\left(t-\mu_{r}\right)^{2}}{2 \sigma_{r}^{2}}}
$$

where $R(i)$ is 1 if trial $i$ was rewarded and 0 otherwise, $\mu_{r}$ is the time of peak reward and $\sigma_{r}$ defines the width of the Gaussian $(0.2 \mathrm{~s}$ in all cases, width chosen to approximate distribution of dopamine activity in response to reward stimuli observed in previous studies (Matsumoto and Hikosaka, 2009; Schultz et al., 1997). For each trial, a value of $\mu_{r}$ was randomly drawn from a uniform distribution spanning $0.2-1.2 \mathrm{~s}$ from the time of the lever press. This distribution was chosen to reflect the $1 \mathrm{~s}$ jitter between lever press and reward used in our behavioral task (see Methods above) as well as the observed delay between reward presentation and peak dopamine release in a variety of studies (Cohen et al., 2012; Matsumoto and Hikosaka, 2009; Parker et al., 2016; Saunders et al., 2018). To ensure that no residual reward response occurred before the time of the lever press, $r_{a}(t)$ was set to 0 for any time $t$ that was $0.2 \mathrm{~s}$ before the time of the peak reward, $\mu_{r}$.

Initial weights. The performance of the model does not depend on the choice of the initial weights as the model learns the correct weights by the end of the first block irrespective of the chosen initial weights. We chose the initial weights to be zero.

Weight and eligibility update implementation. We assumed that the weight and eligibility trace updates start at the time of the simulated nose poke. The nose poke time, relative to the time of the lever press, varies due to a variable delay between the nose poke and the lever presentation as well as variation in time between lever presentation and lever press. To account for this, the weight and eligibility trace updates are initiated at time $t=t_{\text {start, }}$, where $t_{\text {start }}$ was drawn from a Gaussian 
distribution with a mean at $-2.5 \mathrm{~s}$, and a variance of $0.2 \mathrm{~s}$, which was approximately both the time of the nose poke and the time at which choice-selective sequences initiated in the experimental recordings. The eligibility trace is reset to zero at the beginning of each trial. We stopped updating the weights at the end of the trial, defined as 3s after the time of lever press. The eligibility traces were updated according to Equation 16. The weights were updated by integrating Equation 17 with a first-order forward Euler routine. In all simulations, we used a simulation time step $\mathrm{d} t=0.01 \mathrm{~s}$.

\section{Cross-trial analysis of RPE in dopamine neurons}

To generate the regression coefficients in Figure $\mathbf{5 g , l , q}$ and Supplementary Figure $\mathbf{1 0 c}$, we performed a linear regression analysis adapted from (Bayer and Glimcher, 2005), which uses the mouse's reward outcome history from the current and previous 5 trials to predict the average dopamine response to reward feedback on a given trial, $i$ :

$$
D(i)=\beta_{0}+\sum_{j=0}^{5} \beta_{j} \hat{R}(i-j)+\text { error }
$$

Where $D(i)$ is the average dopamine activity from 0.2 to $1.2 \mathrm{~s}$ following reward feedback on trial $i$, $\hat{R}(i-j)$ is the reward outcome history $j$ trials back from trial $i$ ( 1 if $j$ trials back is rewarded and 0 if unrewarded) and $\beta_{j}$ are the calculated regression coefficients which represent the effect of previous outcome $j$ trials back on the strength of the average dopamine activity, $D(i)$. For the regression coefficients generated from recorded dopamine activity (Supplementary Figure 10) we used the Z-scored GCaMP6f fluorescence from VTA-NAc terminal recordings of 11 mice performing the same probabilistic reversal learning task described in this paper (see (Parker et al., 2016) for more details). The regression coefficients for the experimental data as well as the TD model simulations were fit using the LinearRegression function from the linear_model module in Python's scikit-learn package.

\section{Optogenetic stimulation of PL-NAc neurons}

22 male C57BL/6J mice were bilaterally injected in either the $P L(n=14$ mice, $M-L \pm 0.4, A-P 2.0$ and $\mathrm{D}-\mathrm{V}-2.5 \mathrm{~mm})$ or $\mathrm{mTH}(\mathrm{n}=8$ mice, $\mathrm{M}-\mathrm{L} \pm 0.3, \mathrm{~A}-\mathrm{P}-0.7$ and $\mathrm{D}-\mathrm{V}-3.6 \mathrm{~mm}$ ) with $600 \mathrm{nl}$ AAV2/5-CamKIla-hChR2-EYFP (UPenn vector core, injected $0.6 \mu \mathrm{l}$ per hemisphere of titer of $9.6 \times$ $10^{13} \mathrm{pp}$ per $\mathrm{ml}$ ) and optical fibers (300 $\mu \mathrm{m}$ core diameter, $0.37 \mathrm{NA}$ ) delivering 1-2 $\mathrm{mW}$ of $447 \mathrm{~nm}$ laser light (measured at the fiber tip) were implanted bilaterally above the NAc Core at a 10 degree angle $(\mathrm{M}-\mathrm{L} \pm 1.1, \mathrm{~A}-\mathrm{P} 1.4$ and $\mathrm{D}-\mathrm{V}-4.2 \mathrm{~mm})$. An additional cohort of control mice $(\mathrm{n}=8)$ were implanted with optical fibers in the NAc without injection of ChR2 and underwent the same stimulation protocol outlined below (Supplementary Figure 12). Mice were anesthetized for implant surgeries with isoflurane (3-4\% induction and $1-2 \%$ maintenance). Mice were given 5 days of recovery after the surgical procedure before behavioral testing.

During behavioral sessions, 5-ms pulses of $1-3 \mathrm{~mW}, 447-\mathrm{nm}$ blue light was delivered at $20 \mathrm{~Hz}$ on a randomly selected $10 \%$ of trials beginning when the mouse entered the central nose poke. Light stimulation on unrewarded trials ended $1 \mathrm{~s}$ after the end of the CS- presentation. On rewarded trials, light administration ended either 1s after CS+ presentation ('cohort 1') or the end of reward consumption, as measured by the mouse not engaging the reward port for 100ms ('cohort 2'). See Supplementary Figure 13 for a schematic of stimulation times as well as the behavior of the two 
bioRxiv preprint doi: https://doi.org/10.1101/725382; this version posted July 17,2020 . The copyright holder for this preprint (which was not certified by peer review) is the author/funder. All rights reserved. No reuse allowed without permission.

cohorts. Mice alternated between sessions with and without stimulation - sessions without stimulation were excluded from analysis. Anatomical targeting was confirmed as successful in all mice through histology after the experiment, and therefore no mice were excluded from this data set. 


\section{Supplemental Figures}

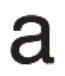

PL, Prelimbic area

ORB, Orbital area

ACA, Anterior cingulate area

ILA, Infralimbic area

500um diameter GRIN lens

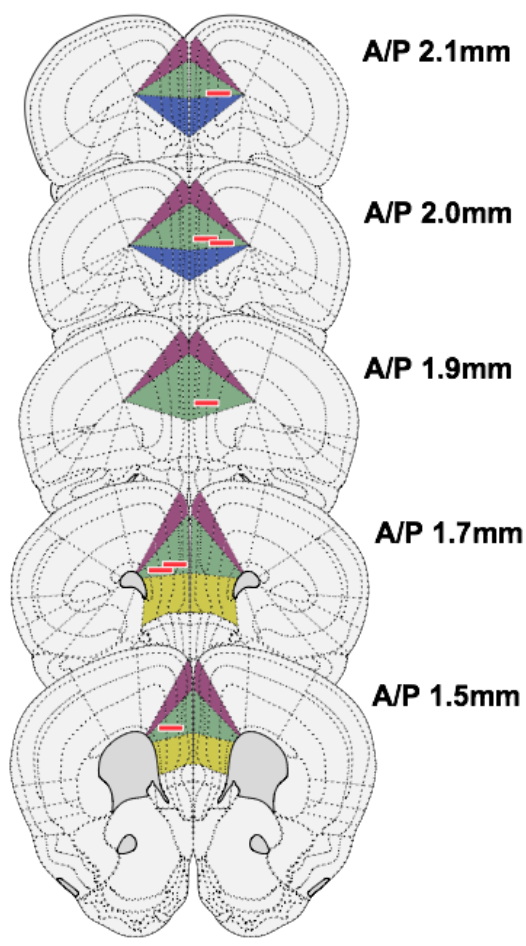

b

MD, Mediodorsal nucleus of the thalamus

PCN, Paracentral nucleus of the thalamus

PVT, Paraventricular nucleus of the thalamus

$\mathrm{CM}$, Central medial nucleus of the thalamus.

PT, Parataenial nucleus of the thalamus

IAD, Interanterodorsal nucleus of the thalamus
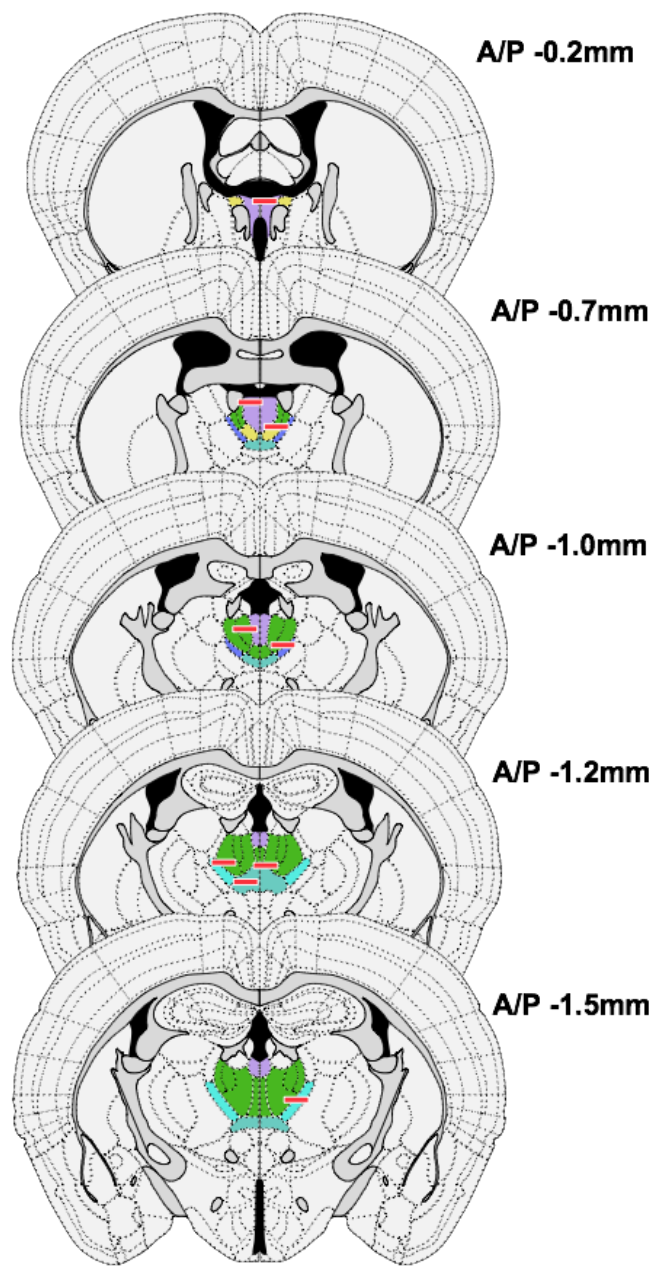

Supplementary Figure 1 - Locations of GRIN lens implants. (a) Schematic of coronal sections along the anterior/posterior axis (A/P, numbers relative to bregma) with recording locations of 7 PL-NAc mice. Red lines indicate bottom of lens implant. (b) Same as a except location of 9 mTH-NAc recordings. 
bioRxiv preprint doi: https://doi.org/10.1101/725382; this version posted July 17,2020 . The copyright holder for this preprint (which was not certified by peer review) is the author/funder. All rights reserved. No reuse allowed without permission.

a
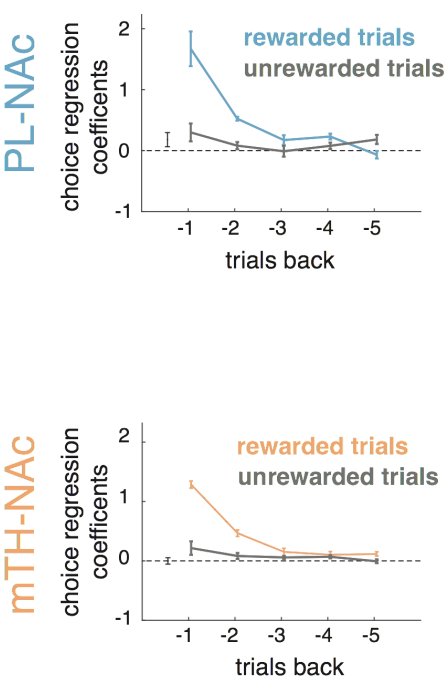

b

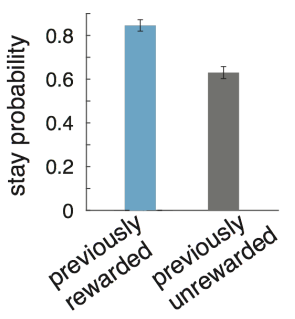

C
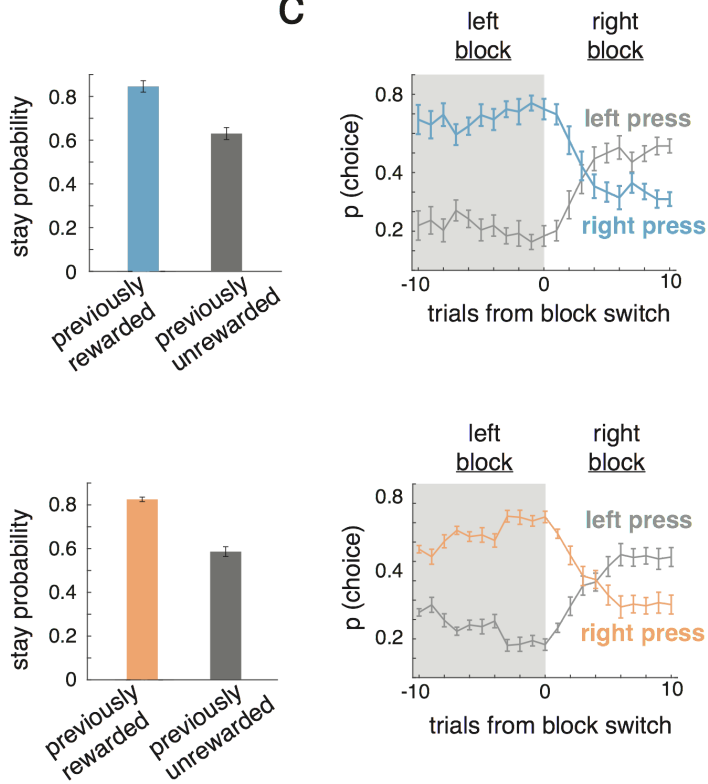

Supplementary Figure 2 - Mice in the PL-NAc and mTH-NAc imaging cohorts have comparable behavior. (a) Top, coefficients from logistic regression to predict choice (see Figure 1) from PL-NAc cohort ( $n=7$ mice). Bottom, same except coefficients from mTH-NAc cohort ( $n=9$ mice). Both cohorts use choice and outcome information from previous trials to predict the current choice. Regression coefficients between the two cohorts are not significantly different for any trials back for either rewarded or unrewarded trials $(P>0.01$, unpaired, two-tailed t-test of regression coefficients across mice at each trial back, $\mathrm{n}=7$ and 9 mice for PL-NAc and mTH-NAc, respectively). (b) Stay probability following rewarded (blue or orange) and unrewarded (grey) trials for PL-NAc (top) and mTH-NAc (bottom) cohorts. Both cohorts have a significantly higher stay probability following a rewarded trial ( $P$ L-NAc: $P=0.00008$; $m$ TH-NAc: $P=0.00003$, paired, two-tailed t-test comparing stay probability on rewarded and unrewarded trials across mice, $n=7$ and 9 mice for PL-NAc and mTH-NAc, respectively). (c) Probability of a left or right lever press following a transition from a left-preferring to right-preferring block of mice from the PL-NAc (top, $n=7$ mice) and $m$ TH-NAc (bottom, $n=9$ mice) cohorts. Both cohorts display a qualitatively similar change in choice behavior following a block transition. In all panels, error bars indicate s.e.m. across mice. 
bioRxiv preprint doi: https://doi.org/10.1101/725382; this version posted July 17, 2020. The copyright holder for this preprint (which was not certified by peer review) is the author/funder. All rights reserved. No reuse allowed without permission.
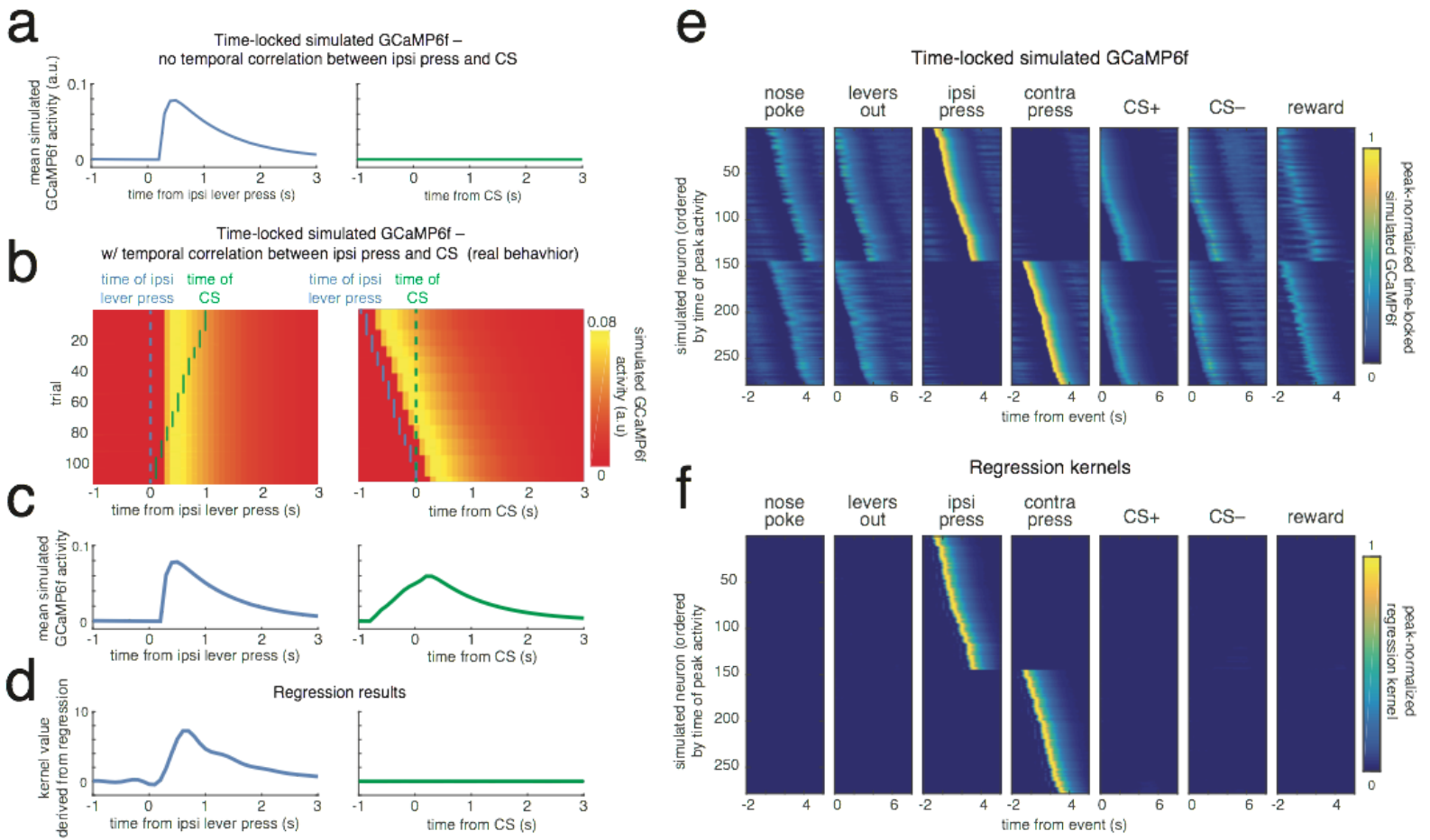

Supplementary Figure 3 - Simulated neural activity to illustrate the ability of the encoding model to successfully relate neural activity to the appropriate behavioral event. (a) Simulated neuron that is responsive only to the ipsilateral lever press. (b) Trial-by-trial heatmap of a simulated neuron that has increased activity time-locked to the ipsilateral lever press. In the data, there was a correlation between the time of lever press and the time of the CS, which produced a time-locked response to the CS, even though the neuron did not respond to that event. Left, activity heatmap aligned to the time of an ipsilateral lever press (dashed blue line) sorted by the time of the subsequent CS presentation (green dots). Right, activity heatmap is aligned to the time of the CS (dashed green line), ordered by the time of the preceding lever press (blue dots). (c) Average activity across trials of the example simulated neuron in b aligned to the lever press, left, and CS presentation, right. Unlike the idealized case in a, when the timing of task events is maintained from the real behavior, the temporal correlations result in a bump in activity aligned to the CS (right plot). Note that this bump in activity is generated entirely by the correlation in event times, since this simulated neuron only had activity in relation to the lever press (and not the CS presentation). (d) Response kernels for lever press, left, and CS, right, derived from the encoding model used to attribute the neural response of individual task events. The model successfully recovers the fact that neural activity in this simulated neuron is related to the lever press and not the CS. (e) Heatmap displaying the average activity from a population of 278 simulated neurons that respond to either the ipsilateral or contralateral lever press, but not the other events. Each neuron responds to the lever press, with a randomly assigned response latency from -1 to 3 seconds. While the strongest average time-locked response is to the ipsilateral or contralateral lever presses, there are visible responses to the other task events as a consequence of the correlation between task events resulting from their temporal proximity. (f) Same as e except heatmap displays the response kernels derived from the encoding model. The model successfully discovers the underlying structure of the data (i.e., that responses are driven by the lever press). 
bioRxiv preprint doi: https://doi.org/10.1101/725382; this version posted July 17,2020 . The copyright holder for this preprint (which was not certified by peer review) is the author/funder. All rights reserved. No reuse allowed without permission.
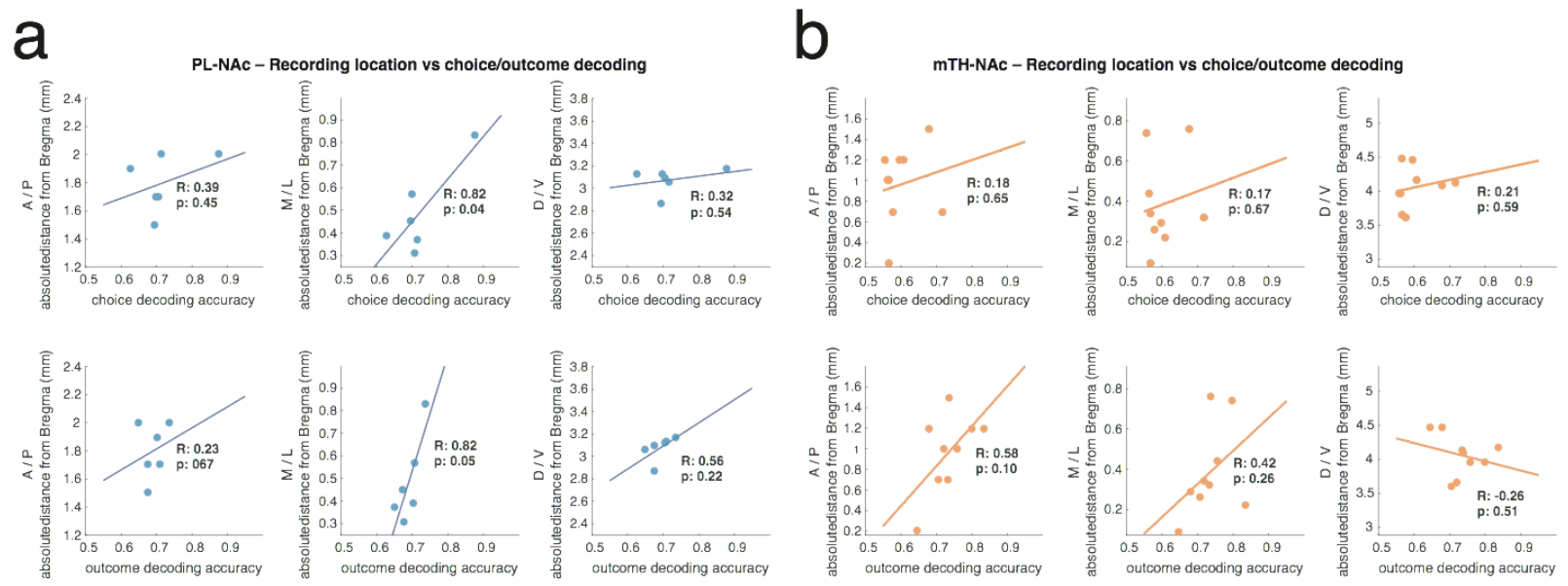

Supplementary Figure 4 - Lack of correlation between recording locations relative to Bregma and choice / outcome decoding. (a) Top, correlations between choice decoding accuracy using recorded PL-NAc activity and, in order, the anterior/posterior (A/P), medial/lateral (M/L) and dorsal/ventral (D/V) recording locations relative to Bregma (see Supp. Fig. 1 for schematic of recording locations; recording locations were aligned to the Allen atlas using the Wholebrain software suite (http://www.wholebrainsoftware.org/) of Furth et al. (2018), see Methods for details). Bottom, same as top except correlation between recording location and outcome decoding accuracy using PL-NAc activity. (b) Same as a except decoding accuracy for choice (top) and outcome (bottom) determined using recorded mTH-NAc activity. All p-values are calculated from Pearson's correlation coefficient; none are significant at the $p<.05$ level after correction for multiple (6) hypotheses using Bonferroni correction. 
bioRxiv preprint doi: https://doi.org/10.1101/725382; this version posted July 17,2020 . The copyright holder for this preprint (which was not certified by peer review) is the author/funder. All rights reserved. No reuse allowed without permission.

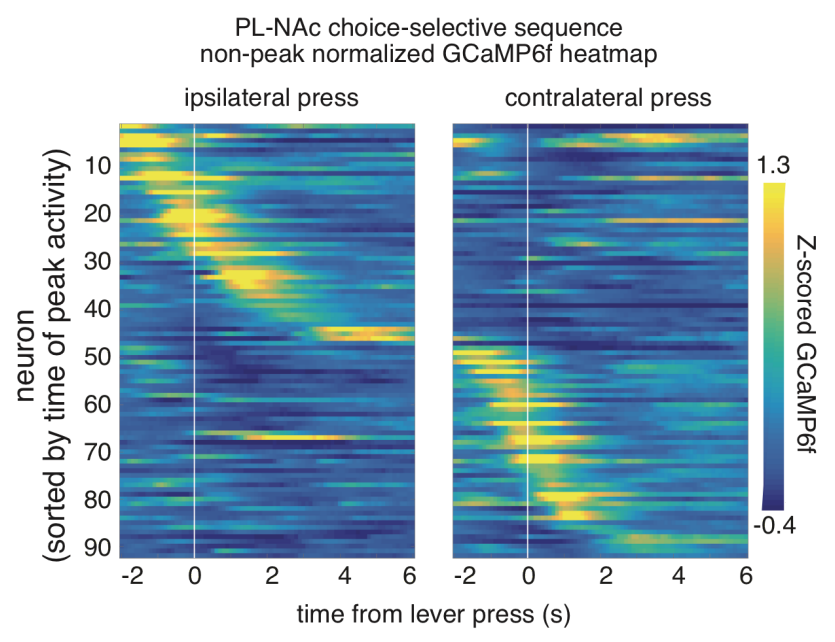

Supplementary Figure 5 - Choice-selective sequences in PL-NAc neurons without peak-normalization. Heatmap demonstrating sequential response of choice-selective PL-NAc neurons to the ipsilateral and contralateral lever press. Similar to Figure $\mathbf{4 b , c}$, but time-locked, trial-averaged GCaMP6f fluorescence is not normalized by the peak response to the lever press and is taken from all trials. 
bioRxiv preprint doi: https://doi.org/10.1101/725382; this version posted July 17, 2020. The copyright holder for this preprint (which was not certified by peer review) is the author/funder. All rights reserved. No reuse allowed without permission.

a

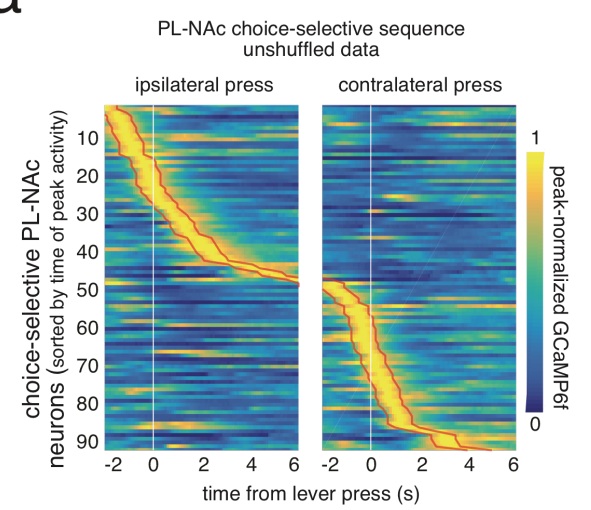

d

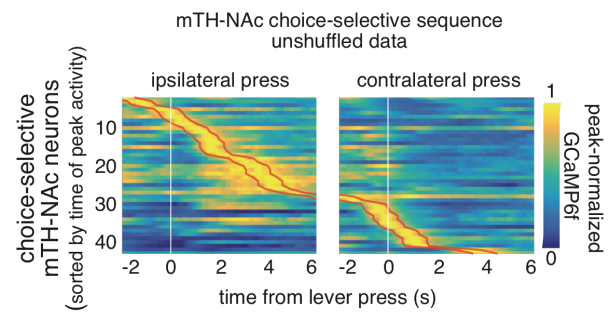

b

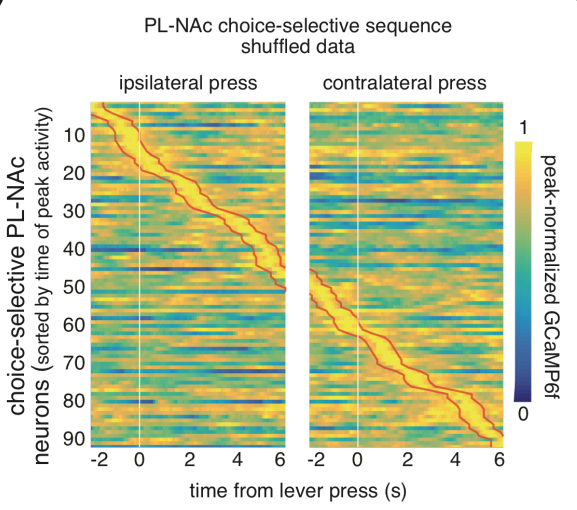

e

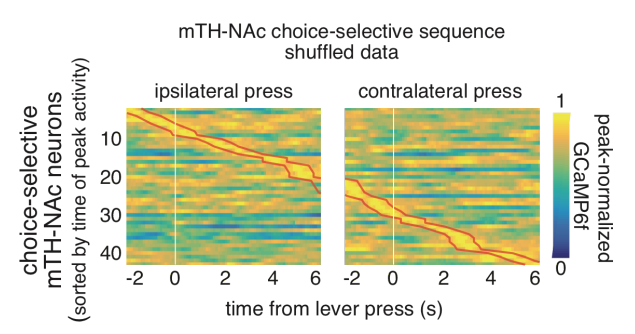

C

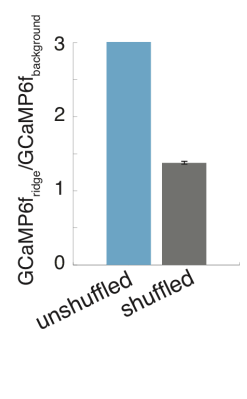

f

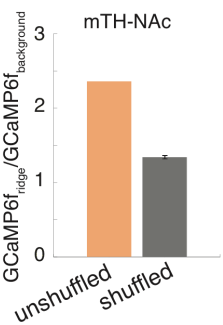

Supplementary Figure 6 - The calculated ridge-to-background ratio of PL-NAc neurons supports the presence of sequences. (a) Sequential activity of PL-NAc choice-selective neurons. Similar to Figure $\mathbf{4 b}$, the heatmap is ordered by the time of peak activity time-locked to the ipsilateral (left column) and contralateral (right column) lever press of each neuron, but instead of cross-validation, activity is averaged across all trials. Red trace represents the borders of the one-second window around the peak defined as the 'ridge'. Activity at all other surrounding timepoints is considered the 'background'. (b) Same as a for data that is shuffled by temporally shifting the GCaMP6f fluorescence trace across a recording session separately for each neuron by a random number of frames, chosen from a uniform distribution. Ordering by the time of peak activity generates spurious sequential activity across the diagonal in shuffled data. (c) Calculated ridge-to-background ratio of PL-NAc neurons using unshuffled (blue) and shuffled data (grey). A ratio is calculated for each individual neuron and the average of these ratios across all neurons displayed in the heatmap is shown. The ratio calculated from unshuffled data is significantly larger than that from the shuffled data $(P<0.0001$, comparison between unshuffled data and distribution of 500 shuffled iterations). Error bars for shuffled data indicate one standard deviation. (d-f) Same as a-c but ridge-to-background is calculated using mTH-NAc neural recordings. Similar to PL-NAc, the ratio calculated from unshuffled data was significantly larger than that from the shuffled data $(P<0.0001)$. However, when comparing across the populations, the ridge-to-background calculated using PL-NAc neurons (3.01+/-0.12, mean+/-sem, $n=92$ neurons) was significantly larger than that using mTH-NAc $(2.36+/-0.12$, mean+/-sem, $n=42$ neurons; $\mathrm{P}=0.001$ : unpaired, two-tailed t-test comparing ratio between PL-NAc and mTH-NAc neurons, n=92 and 42 for PL-NAc and mTH-NAc populations, respectively). 
bioRxiv preprint doi: https://doi.org/10.1101/725382; this version posted July 17, 2020. The copyright holder for this preprint (which was not certified by peer review) is the author/funder. All rights reserved. No reuse allowed without permission.

a

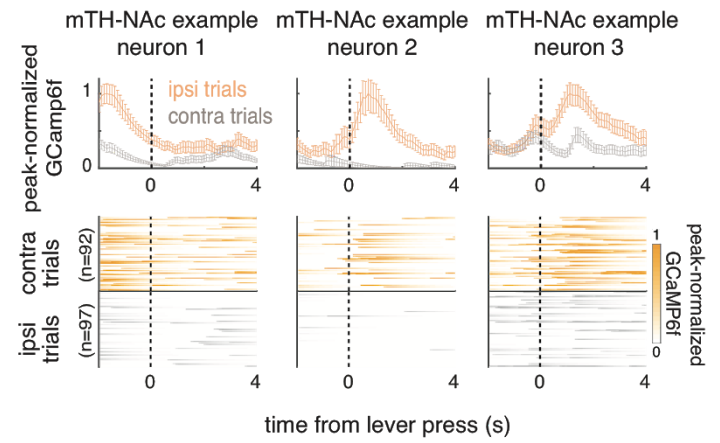

b

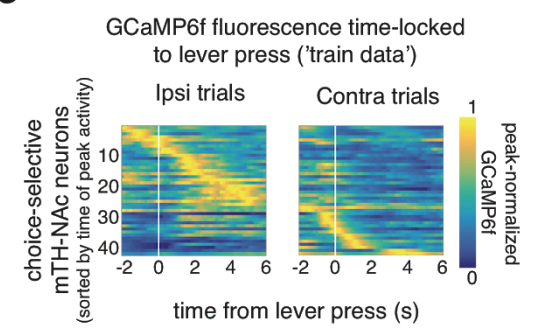

C

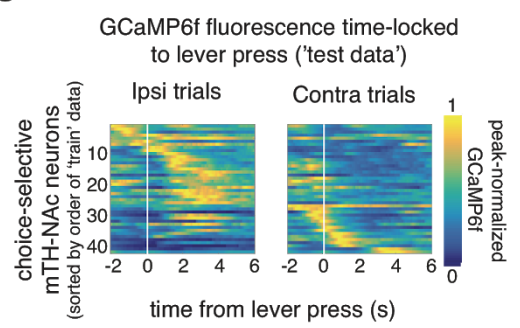

d

peak GCaMP6f response time relative to lever press - train vs test data

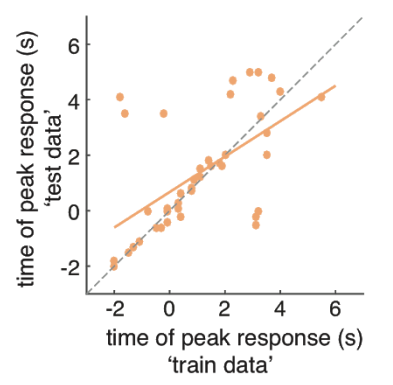

e

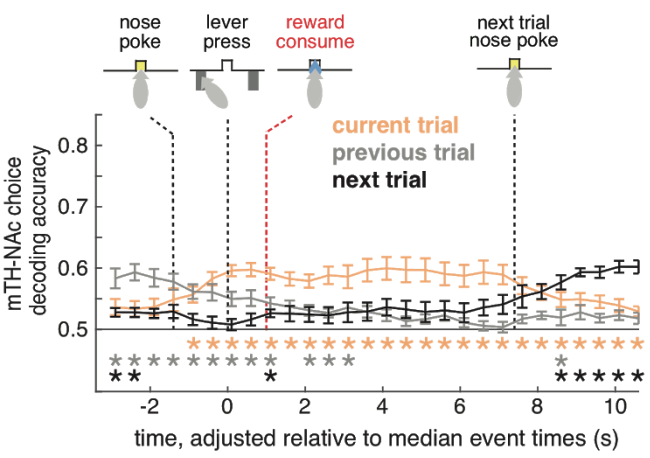

Supplementary Figure 7 - mTH-NAc choice-selective neurons display sequential activity that is less consistent than PL-NAc. (a) Top; average GCaMP6f fluorescence of three simultaneously imaged mTH-NAc choice-selective neurons with different response times relative to the lever press. Error bars are s.e.m across trials. Bottom, heatmaps of GCaMP6f fluorescence response across trials to ipsilateral (orange) and contralateral (grey) lever presses. (b,c) Heatmaps of choice-selective mTH-NAc neurons' peak-normalized GCaMP6f response to lever press ( $n=42 / 256$ neurons). Each row is the average GCaMP6f fluorescence time-locked to the ipsilateral (left column) and contralateral (right column) lever press for a neuron, normalized by the neuron's peak average fluorescence. In $\mathbf{b}$ ('train data'), heatmap is generated using a randomly selected half of trials and ordered by the time of each neuron's peak activity. In c ('test data'), the peak-normalized, time-locked GCaMP6f fluorescence from the other half of trials was used while maintaining the order from 'train data' in $\mathbf{b}$. Compare to PL-NAc data in Figure 4c. (d) Correlation between the time of peak activity using the 'train' (horizontal axis) and 'test' (vertical axis) trials for choice-selective mTH-NAc neurons. While mTH-NAc choice-selective neurons also show significant correlation between 'train' and 'test' trials $\left(R^{2}=0.59, P=4.2 \times 10^{-5}, n=42\right.$ neurons from 9 mice), this correlation is significantly lower than that of PL-NAc (comparison with data in Figure 4c; $P=0.0096, Z=2.34$, Fisher's R-to-Z transformation, comparison of correlation coefficients derived from comparing peak activity between 'test' and 'training' data from PL-NAc versus mTH-NAc). (e) Average choice decoding accuracy of the mice's choice on the current (orange), previous (grey) and next trial (black) as a function of GCaMP6f fluorescence throughout the current trial. GCaMP6f fluorescence is taken from 100 random selections per mouse of 10 simultaneously imaged mTH-NAc neurons (each trial's activity is adjusted in a piecewise linear manner relative to the median time of the nose poke, lever press and next trial nose poke, see Methods for details). Error bars are s.e.m. across mice ( $n=9$ mice). Red dashed line indicates median onset of reward consumption. Asterisks indicate significant decoding accuracy above chance, $\mathrm{P}<0.01$, two-tailed, one-sample t-test across mice. 
bioRxiv preprint doi: https://doi.org/10.1101/725382; this version posted July 17,2020 . The copyright holder for this preprint (which was not certified by peer review) is the author/funder. All rights reserved. No reuse allowed without permission.

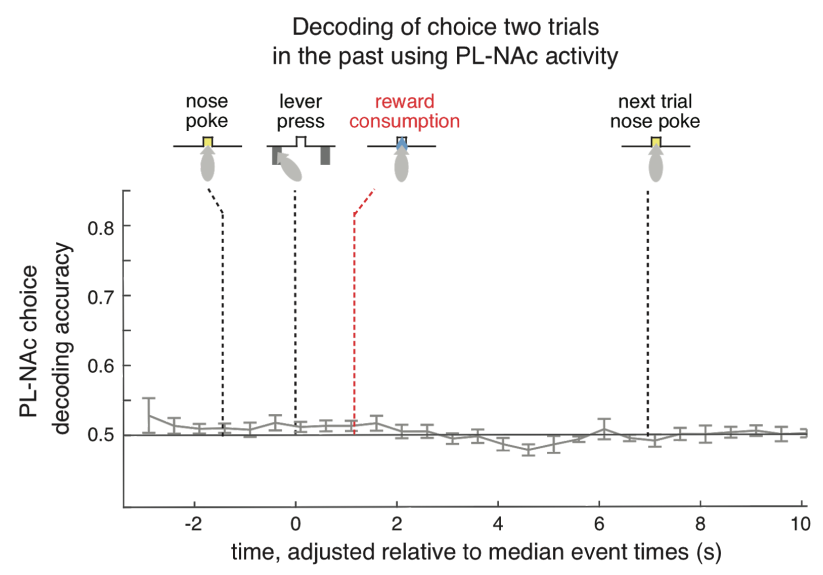

Supplementary Figure 8 - Choice identity two trials in the past cannot be accurately decoded using PL-NAc activity. Decoding accuracy for the mice's choice two trials back using activity from 10 simultaneously recorded PL-NAc neurons. Unlike choice decoding on the current and previous trial (Figure 4e; blue and black traces, respectively), PL-NAc activity is not able to accurately decode choice from two trials back at any time point in the trial $(\mathrm{P}>0.05$ for all time points: one-sample, two-tailed t-test across mice comparing decoding accuracy with chance rate of 0.5 ). 
bioRxiv preprint doi: https://doi.org/10.1101/725382; this version posted July 17, 2020. The copyright holder for this preprint (which was not certified by peer review) is the author/funder. All rights reserved. No reuse allowed without permission.

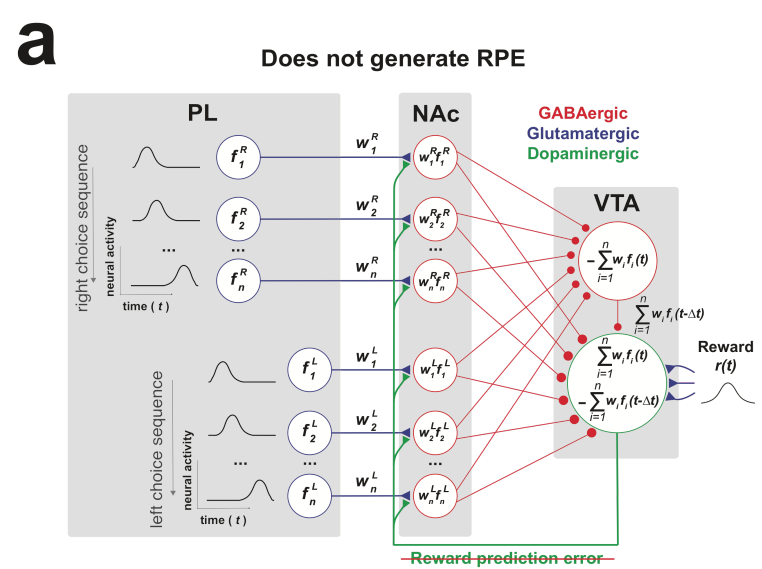

b

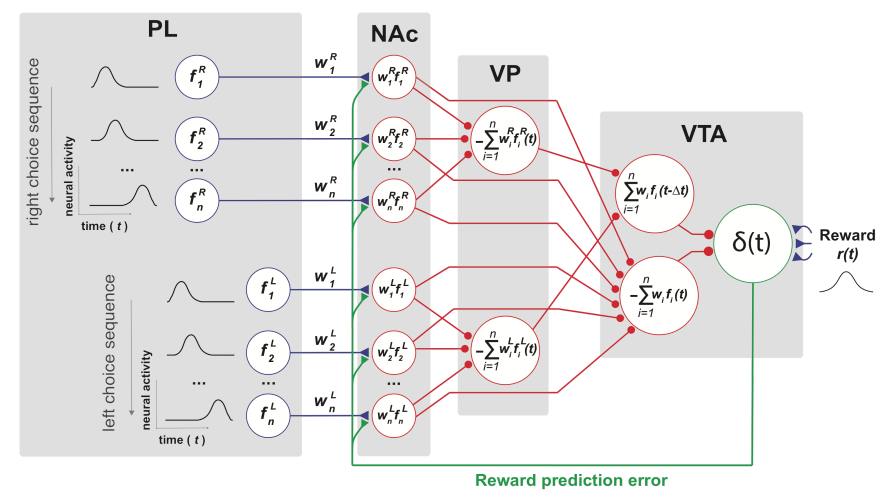

C
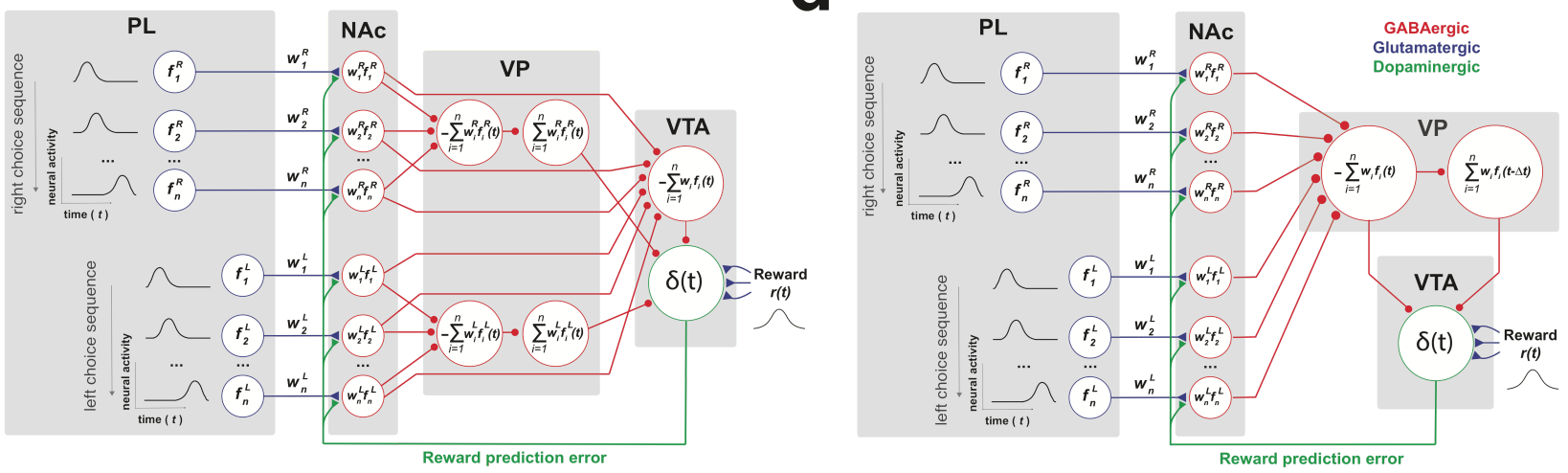

Supplementary Figure 9 - Alternative model architectures used to implement TD learning. (a-f) Alternative models constructed using known circuit architecture. All models except a are able to correctly generate an RPE signal by providing a 'fast excitatory' and 'slow-inhibitory' value pathway to VTA dopamine neuron. Note that all model variants rely on choice-selective sequences in PL-NAc to bridge actions and outcomes across time. For all model variants, GABAergic, glutamatergic and dopaminergic projections are denoted as red, blue and green, respectively. Brain region abbreviations are: prelimbic cortex, PL; nucleus accumbens, NAc; ventral pallidum, VP; ventral tegmental area, VTA. (a) To account for previous work describing direct projections from NAc D1R neurons to the VTA (Beier et al., 2015; Watabe-Uchida et al., 2012; Yang et al., 2018), this alternative model architecture has NAc neurons projecting directly to the VTA, skipping the VP. However, this architecture does not produce an RPE signal in dopamine neurons because of a sign error (rather than a fast excitatory value and delayed inhibitory value, VTA dopamine neurons receive the opposite signal). Additionally, this circuit architecture is inconsistent with previous studies indicating that NAc D1R neurons project primarily to the VTA GABA neurons, rather than sending balanced projections to the VTA dopamine and GABA neurons (Bocklisch et al., 2013). (b) In this model, the fast excitatory pathway is generated via a direct projection of NAc neurons onto the VTA GABA neuron while the slow inhibitory pathway passes through the VP before synapsing onto a VTA GABA neuron. (c) Similar to b except that the slow inhibitory pathway contains an additional VP neuron, which accomplishes the sign inversion and delay assigned to a VTA GABA neuron in $\mathbf{b}$. Since the models in $\mathbf{b} \& \mathbf{c}$ prescribe a role for the observed NAc-D1R projections to VTA GABA neurons, they produce negative value signals in VTA GABA neurons, whereas only positive value signals have been observed experimentally in identified GABA interneurons in the VTA (Cohen et al., 2012). (d) In this model, the delay and inversion of the value signal is accomplished through a second VP neuron as opposed to the VTA GABA neuron in Figure 5a. This generates both positive and negative value encoding neurons in the VP, as has been observed in experimental recordings (Stephenson-Jones et al., 2020; Tian et al., 2016) In addition, this model illustrates that VP neurons do not need to convey separate value signals for left and right choices, but can instead sum the value signals from NAc into a single combined value signal. 
a

VTA-NAc DA response rewarded trials

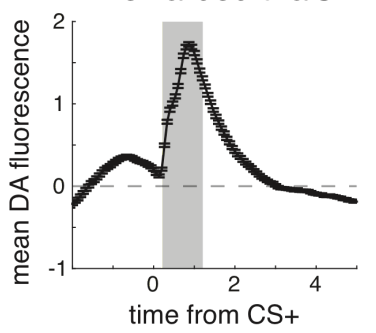

b

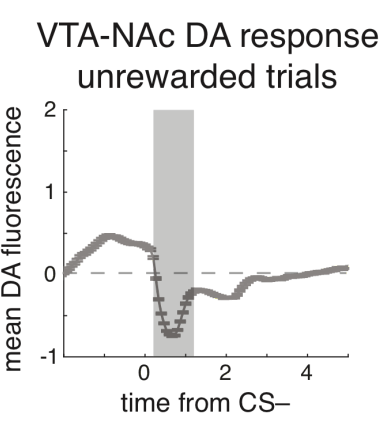

C

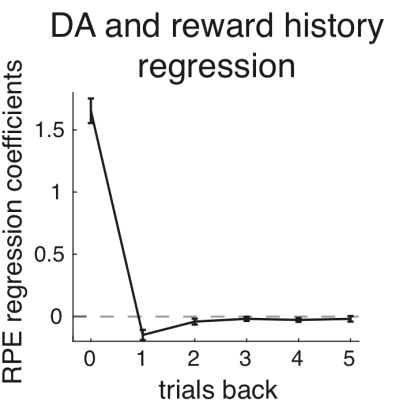

Supplementary Figure 10 - Reward prediction error (RPE) encoding observed in recorded dopamine (DA) activity is similar to that produced by our TD model. (a) Mean bulk GCaMP6f fluorescence from VTA-NAc DA terminals in response to a conditioned stimulus signaling reward (CS+, data taken from (Parker et al., 2016). (b) Same as a except DA fluorescence in response to the conditioned stimulus signaling an unrewarded trial (CS-). (c) Coefficients from a linear regression similar to that in Figure $\mathbf{5 g}, \mathbf{l}$, except outcome is predicted using mean DA fluorescence signals from $0.2-1.2 \mathrm{~s}$ relative to the time of CS presentation (see shaded region in $\mathbf{a , b}$ ). The positive coefficient for the current trial and negative coefficients for previous trials indicate the encoding of an RPE. Error bars represent s.e.m across 11 recording sites. 


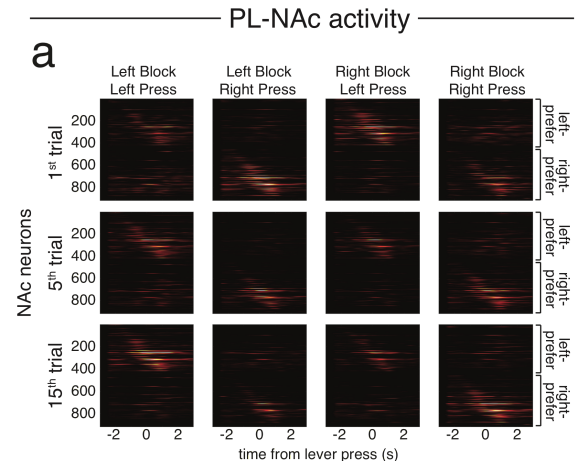

b

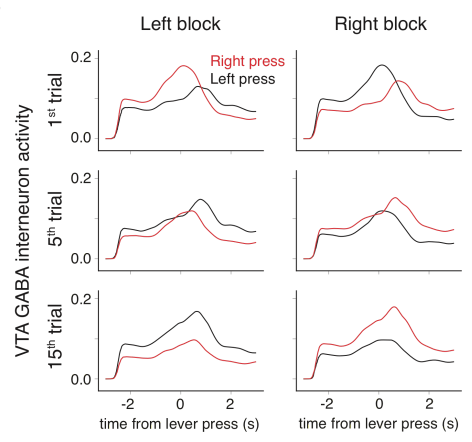

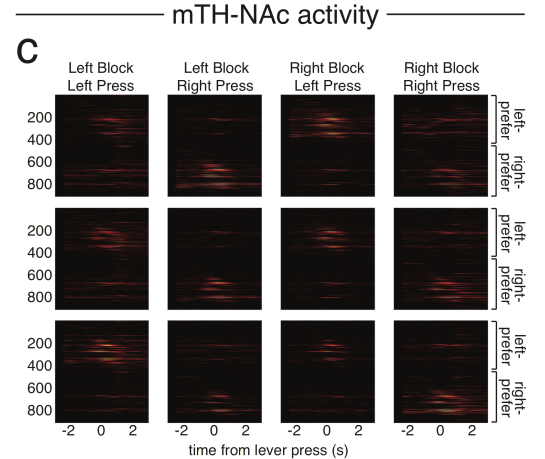

d

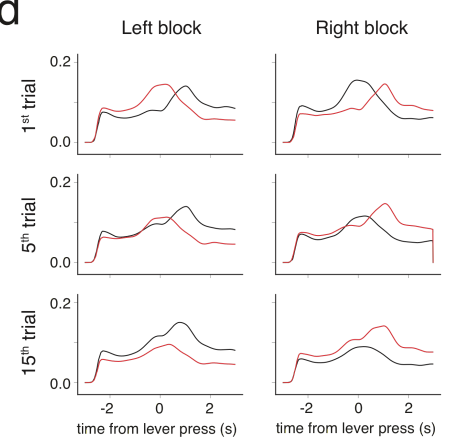

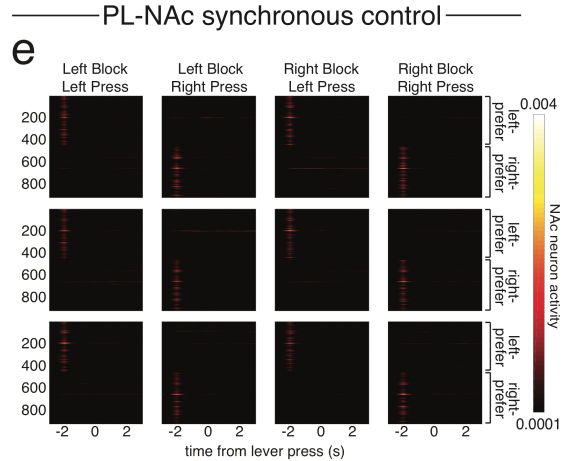

f

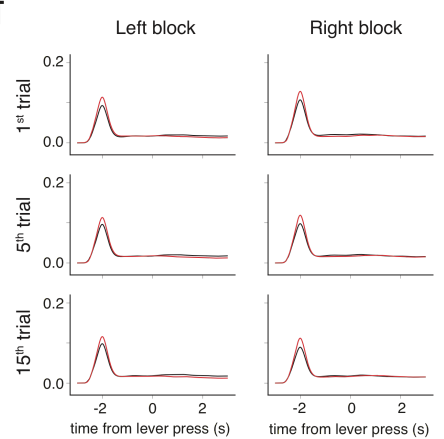

Supplementary Figure 11 - TD model using sequential but not synchronous PL-NAc activity correctly modulates activity in NAc projection neurons and VTA GABA interneurons. (a) Heatmaps of average activity relative to the time of the lever press for NAc projection neurons in the PL-NAc TD model (Figure 5c). Top, middle and bottom heatmaps are the average activity across the first, fifth and fifteenth trial of each block, respectively. Each column is the average activity across trials from different block/press combinations. For each subplot, the left-preferring neurons are ordered first, and the right preferring are ordered second. The activity of these left- and right-preferring NAc neurons increases throughout a block of their respective lever preference. In contrast, their activity decreases throughout a block opposite to their lever preference. (b) Average activity of VTA GABA interneuron from TD model using PL-NAc activity as input on left (black) or right (red) trials. Activity is relative to the time of the lever press across the first, fifth and fifteenth trials of a left-preferring (left column) or right-preferring (right column) block. Similar to a, throughout a left block (left column), the activity on left press trials increases from the first to fifteenth trial while activity on right presses decreases. The opposite pattern is seen for left and right press trials throughout a right block (right column). (c,d) Same as a,b except NAc and VTA GABA interneuron generated using mTH-NAc as input to the TD model. $(\mathbf{e}, \mathbf{f})$ Same as $\mathbf{a}, \mathbf{b}$ except NAc and VTA GABA interneuron from the PL-NAc synchronous control TD model. 
a

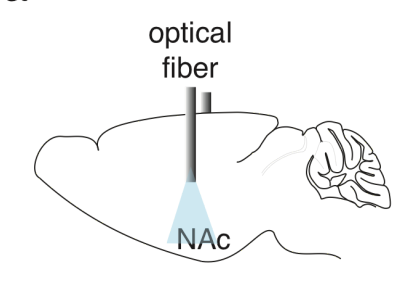

C

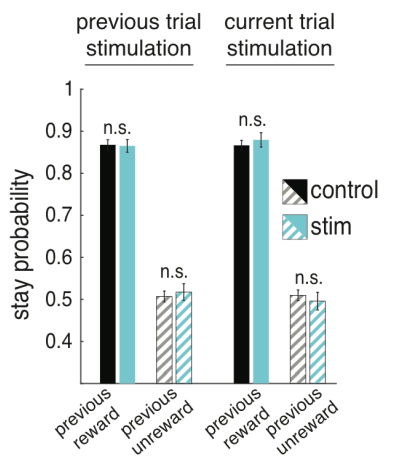

b

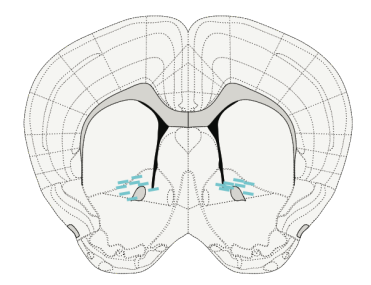

d

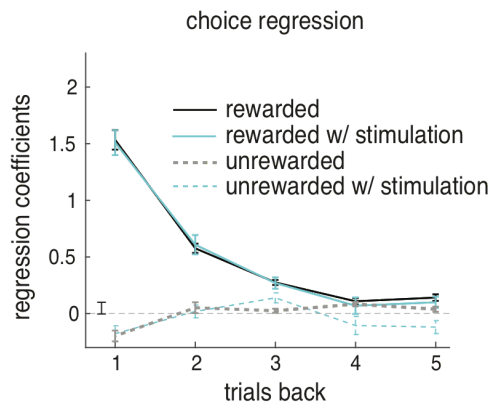

Supplementary Figure 12 - Laser stimulation in control mice that do not express opsin has no effect on behavior. (a) Surgical schematic of light-only control cohort. Optical fibers were implanted into the NAc. (b) Optical fiber tip locations of light-only control cohort ( $n=8$ mice). (c) Unlike PL-NAc ChR2 expressing mice (Figure 6f,g), neither current nor previous trial stimulation changed the stay probability in control mice following rewarded $(P=0.52$ : previous trial stimulation; $P=0.58$ : current trial stimulation; paired t-test) or unrewarded trials $(P=0.24$ : previous trial stimulation; $P=0.47$ : current trial stimulation; paired t-test). (d) Likewise, stimulation from multiple trials back had no effect on choice $(P>0.05$ for all trials back, $t$-test across mice's laser $x$ choice interaction term coefficients). 
bioRxiv preprint doi: https://doi.org/10.1101/725382; this version posted July 17,2020 . The copyright holder for this preprint (which was not certified by peer review) is the author/funder. All rights reserved. No reuse allowed without permission.

a

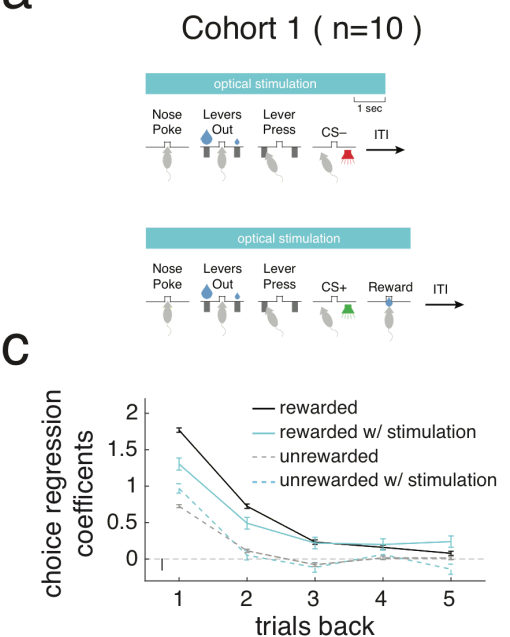

b

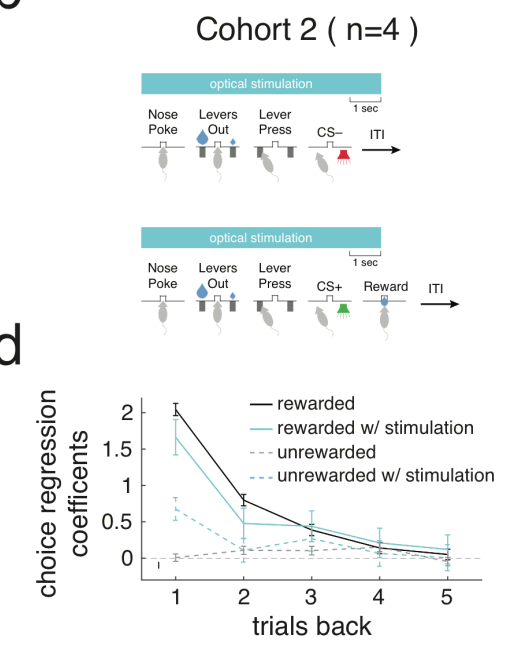

Supplementary Figure 13 - Effect of PL-NAc optogenetic stimulation in two cohorts. (a) Schematic of optical stimulation parameters for cohort 1 . On $10 \%$ of unrewarded trials, optical stimulation began when the mouse entered the central nosepoke and ended 1s after the end of the $500 \mathrm{~ms}$ CS- tone. On $10 \%$ of rewarded trials, stimulation began with nose poke and ended after the mouse left the reward port. (b) Schematic for cohort 2. Unlike cohort 1, optical stimulation ended on the same timescale on both rewarded and unrewarded trials, 1s after the end of CS presentation. (c) Logistic regression model similar to that in Figure 1e demonstrating the effect of PL-NAc stimulation on lever choice in cohort 1 mice ( $n=10$ mice, see Methods for model details). Rewarded trials with stimulation one and two trials back decreased stay probability compared with rewarded trials without stimulation. Stimulation had an opposite effect on unrewarded trials, for which there was an increase in stay probability following stimulation one trial back compared to trials without stimulation. (d) Same as c except data from cohort 2 ( $n=4$ mice). Effect of optical stimulation of PL-NAc neurons was qualitatively similar across the two cohorts. 


\section{References}

Aggarwal, M., Hyland, B.I., and Wickens, J.R. (2012). Neural control of dopamine neurotransmission: implications for reinforcement learning. Eur. J. Neurosci. 35, 1115-1123.

Akhlaghpour, H., Wiskerke, J., Choi, J.Y., Taliaferro, J.P., Au, J., and Witten, I.B. (2016). Dissociated sequential activity and stimulus encoding in the dorsomedial striatum during spatial working memory. Elife 5, e19507.

Apicella, P., Ljungberg, T., Scarnati, E., and Schultz, W. (1991). Responses to reward in monkey dorsal and ventral striatum. Experimental Brain Research 85.

Asaad, W.F., Lauro, P.M., Perge, J.A., and Eskandar, E.N. (2017). Prefrontal Neurons Encode a Solution to the Credit-Assignment Problem. The Journal of Neuroscience 37, 6995-7007.

Atallah, H.E., Lopez-Paniagua, D., Rudy, J.W., and O'Reilly, R.C. (2007). Separate neural substrates for skill learning and performance in the ventral and dorsal striatum. Nat. Neurosci. 10, 126-131.

Bari, B.A., Grossman, C.D., Lubin, E.E., Rajagopalan, A.E., Cressy, J.I., and Cohen, J.Y. (2019). Stable Representations of Decision Variables for Flexible Behavior. Neuron 103, 922-933.e7.

Bayer, H.M., and Glimcher, P.W. (2005). Midbrain Dopamine Neurons Encode a Quantitative Reward Prediction Error Signal. Neuron 47, 129-141.

Beier, K.T., Steinberg, E.E., DeLoach, K.E., Xie, S., Miyamichi, K., Schwarz, L., Gao, X.J., Kremer, E.J., Malenka, R.C., and Luo, L. (2015). Circuit Architecture of VTA Dopamine Neurons Revealed by Systematic Input-Output Mapping. Cell 162, 622-634.

Bocklisch, C., Pascoli, V., Wong, J.C.Y., House, D.R.C., Yvon, C., de Roo, M., Tan, K.R., and Lüscher, C. (2013). Cocaine disinhibits dopamine neurons by potentiation of GABA transmission in the ventral tegmental area. Science 341, 1521-1525.

Boulougouris, V., Castañé, A., and Robbins, T.W. (2009). Dopamine D2/D3 receptor agonist quinpirole impairs spatial reversal learning in rats: investigation of D3 receptor involvement in persistent behavior. Psychopharmacology 202, 611-620.

Britt, J.P., Benaliouad, F., McDevitt, R.A., Stuber, G.D., Wise, R.A., and Bonci, A. (2012). Synaptic and Behavioral Profile of Multiple Glutamatergic Inputs to the Nucleus Accumbens. Neuron 76, 790-803.

Brog, J.S., Salyapongse, A., Deutch, A.Y., and Zahm, D.S. (1993). The patterns of afferent innervation of the core and shell in the "accumbens" part of the rat ventral striatum: immunohistochemical detection of retrogradely transported fluoro-gold. J. Comp. Neurol. 338, 255-278.

Cador, M., Robbins, T.W., and Everitt, B.J. (1989). Involvement of the amygdala in stimulus-reward associations: interaction with the ventral striatum. Neuroscience $30,77-86$.

Cameron, C.M., Murugan, M., Choi, J.Y., Engel, E.A., and Witten, I.B. (2019). Increased Cocaine Motivation Is Associated with Degraded Spatial and Temporal Representations in IL-NAc Neurons. Neuron 103, 80-91.e7.

Campus, P., Covelo, I.R., Kim, Y., Parsegian, A., Kuhn, B.N., Lopez, S.A., Neumaier, J.F., Ferguson, S.M., Solberg Woods, L.C., Sarter, M., et al. (2019). The paraventricular thalamus is a critical 
mediator of top-down control of cue-motivated behavior in rats. Elife 8 .

Cardinal, R.N., and Cheung, T.H.C. (2005). Nucleus accumbens core lesions retard instrumental learning and performance with delayed reinforcement in the rat. BMC Neurosci. 6, 9.

Carelli, R.M., King, V.C., Hampson, R.E., and Deadwyler, S.A. (1993). Firing patterns of nucleus accumbens neurons during cocaine self-administration in rats. Brain Research 626, 14-22.

Carrillo-Reid, L., Tecuapetla, F., Tapia, D., Hernández-Cruz, A., Galarraga, E., Drucker-Colin, R., and Bargas, J. (2008). Encoding network states by striatal cell assemblies. J. Neurophysiol. 99, 1435-1450.

Centonze, D., Picconi, B., Gubellini, P., Bernardi, G., and Calabresi, P. (2001). Dopaminergic control of synaptic plasticity in the dorsal striatum. European Journal of Neuroscience 13, 1071-1077.

Chen, R., Puzerey, P.A., Roeser, A.C., Riccelli, T.E., Podury, A., Maher, K., Farhang, A.R., and Goldberg, J.H. (2019). Songbird ventral pallidum sends diverse performance error signals to dopaminergic midbrain. Neuron 103, 266-276.e4.

Cohen, J.Y., Haesler, S., Vong, L., Lowell, B.B., and Uchida, N. (2012). Neuron-type-specific signals for reward and punishment in the ventral tegmental area. Nature 482, 85-88.

Collins, A.G.E., and Frank, M.J. (2012). How much of reinforcement learning is working memory, not reinforcement learning? A behavioral, computational, and neurogenetic analysis. European Journal of Neuroscience 35, 1024-1035.

Collins, A.G.E., Brown, J.K., Gold, J.M., Waltz, J.A., and Frank, M.J. (2014). Working memory contributions to reinforcement learning impairments in schizophrenia. J. Neurosci. 34, 13747-13756.

Collins, A.G.E., Ciullo, B., Frank, M.J., and Badre, D. (2017). Working Memory Load Strengthens Reward Prediction Errors. J. Neurosci. 37, 4332-4342.

Collins, A.L., Aitken, T.J., Huang, I.-W., Shieh, C., Greenfield, V.Y., Monbouquette, H.G., Ostlund, S.B., and Wassum, K.M. (2019). Nucleus accumbens cholinergic interneurons oppose cue-motivated behavior. Biol. Psychiatry 86, 388-396.

Cox, J., and Witten, I.B. (2019). Striatal circuits for reward learning and decision-making. Nature Reviews Neuroscience 20, 482-494.

Day, J.J., and Carelli, R.M. (2007). The nucleus accumbens and Pavlovian reward learning. The Neuroscientist 13, 148-159.

Day, J.J., Wheeler, R.A., Roitman, M.F., and Carelli, R.M. (2006). Nucleus accumbens neurons encode Pavlovian approach behaviors: evidence from an autoshaping paradigm. Eur. J. Neurosci. 23, 1341-1351.

Dayan, P., and Niv, Y. (2008). Reinforcement learning: the good, the bad and the ugly. Curr. Opin. Neurobiol. 18, 185-196.

Di Ciano, P., Cardinal, R.N., Cowell, R.A., Little, S.J., and Everitt, B.J. (2001). Differential involvement of NMDA, AMPA/kainate, and dopamine receptors in the nucleus accumbens core in the acquisition and performance of pavlovian approach behavior. J. Neurosci. 21, 9471-9477. 
Ding, X., Qiao, Y., Piao, C., Zheng, X., Liu, Z., and Liang, J. (2014). N-methyl-D-aspartate receptor-mediated glutamate transmission in nucleus accumbens plays a more important role than that in dorsal striatum in cognitive flexibility. Front. Behav. Neurosci. 8, 304.

Do-Monte, F.H., Minier-Toribio, A., Quiñones-Laracuente, K., Medina-Colón, E.M., and Quirk, G.J. (2017). Thalamic regulation of sucrose seeking during unexpected reward omission. Neuron 94 , 388-400.e4.

Doya, K. (2002). Metalearning and neuromodulation. Neural Netw. 15, 495-506.

Engelhard, B., Finkelstein, J., Cox, J., Fleming, W., Jang, H.J., Ornelas, S., Koay, S.A., Thiberge, S.Y., Daw, N.D., Tank, D.W., et al. (2019). Specialized coding of sensory, motor and cognitive variables in VTA dopamine neurons. Nature 570, 509-513.

Eshel, N., Bukwich, M., Rao, V., Hemmelder, V., Tian, J., and Uchida, N. (2015). Arithmetic and local circuitry underlying dopamine prediction errors. Nature 525, 243-246.

Everitt, B.J., Morris, K.A., O'Brien, A., and Robbins, T.W. (1991). The basolateral amygdala-ventral striatal system and conditioned place preference: further evidence of limbic-striatal interactions underlying reward-related processes. Neuroscience 42, 1-18.

Fee, M.S., and Goldberg, J.H. (2011). A hypothesis for basal ganglia-dependent reinforcement learning in the songbird. Neuroscience 198, 152-170.

Fisher, S.D., Robertson, P.B., Black, M.J., Redgrave, P., Sagar, M.A., Abraham, W.C., and Reynolds, J.N.J. (2017). Reinforcement determines the timing dependence of corticostriatal synaptic plasticity in vivo. Nat. Commun. 8, 334.

Fürth, D., Vaissière, T., Tzortzi, O., Xuan, Y., Märtin, A., Lazaridis, I., Spigolon, G., Fisone, G., Tomer, R., Deisseroth, K., et al. (2018). An interactive framework for whole-brain maps at cellular resolution. Nat. Neurosci. 21, 139-149.

Genovesio, A., Brasted, P.J., and Wise, S.P. (2006). Representation of future and previous spatial goals by separate neural populations in prefrontal cortex. J. Neurosci. 26, 7305-7316.

Gerfen, C.R., and Surmeier, D.J. (2011). Modulation of striatal projection systems by dopamine. Annu. Rev. Neurosci. 34, 441-466.

Gersch, T.M., Foley, N.C., Eisenberg, I., and Gottlieb, J. (2014). Neural Correlates of Temporal Credit Assignment in the Parietal Lobe.

Gershman, S.J., Moustafa, A.A., and Ludvig, E.A. (2014). Time representation in reinforcement learning models of the basal ganglia. Front. Comput. Neurosci. 7, 194.

Gerstner, W., Lehmann, M., Liakoni, V., Corneil, D., and Brea, J. (2018). Eligibility Traces and Plasticity on Behavioral Time Scales: Experimental Support of NeoHebbian Three-Factor Learning Rules. Front. Neural Circuits 12, 53.

Gläscher, J., Hampton, A.N., and O’Doherty, J.P. (2009). Determining a Role for Ventromedial Prefrontal Cortex in Encoding Action-Based Value Signals During Reward-Related Decision Making. Cereb. Cortex 19, 483-495.

Grabenhorst, F., and Rolls, E.T. (2011). Value, pleasure and choice in the ventral prefrontal cortex. 
Trends Cogn. Sci. 15, 56-67.

Groenewegen, H.J., Becker, N.E., and Lohman, A.H. (1980). Subcortical afferents of the nucleus accumbens septi in the cat, studied with retrograde axonal transport of horseradish peroxidase and bisbenzimid. Neuroscience 5, 1903-1916.

Haber, S.N., and Knutson, B. (2010). The reward circuit: linking primate anatomy and human imaging. Neuropsychopharmacology 35, 4-26.

Haber, S.N., Fudge, J.L., and McFarland, N.R. (2000). Striatonigrostriatal pathways in primates form an ascending spiral from the shell to the dorsolateral striatum. The Journal of Neuroscience 20, 2369-2382.

Hamid, A.A., Pettibone, J.R., Mabrouk, O.S., Hetrick, V.L., Schmidt, R., Vander Weele, C.M., Kennedy, R.T., Aragona, B.J., and Berke, J.D. (2016). Mesolimbic dopamine signals the value of work. Nat. Neurosci. 19, 117-126.

Harvey, C.D., Coen, P., and Tank, D.W. (2012). Choice-specific sequences in parietal cortex during a virtual-navigation decision task. Nature 484, 62-68.

Hazy, T.E., Frank, M.J., and O'Reilly, R.C. (2010). Neural mechanisms of acquired phasic dopamine responses in learning. Neurosci. Biobehav. Rev. 34, 701-720.

Hernandez, P.J., Sadeghian, K., and Kelley, A.E. (2002). Early consolidation of instrumental learning requires protein synthesis in the nucleus accumbens. Nat. Neurosci. 5, 1327-1331.

Hjelmstad, G.O. (2004). Dopamine excites nucleus accumbens neurons through the differential modulation of glutamate and GABA release. J. Neurosci. 24, 8621-8628.

Hunnicutt, B.J., Jongbloets, B.C., Birdsong, W.T., Gertz, K.J., Zhong, H., and Mao, T. (2016). A comprehensive excitatory input map of the striatum reveals novel functional organization. Elife 5 .

Ikeda, H., Saigusa, T., Kamei, J., Koshikawa, N., and Cools, A.R. (2013). Spiraling dopaminergic circuitry from the ventral striatum to dorsal striatum is an effective feed-forward loop. Neuroscience $241,126-134$.

Ito, M., and Doya, K. (2015). Parallel Representation of Value-Based and Finite State-Based Strategies in the Ventral and Dorsal Striatum. PLoS Comput. Biol. 11, e1004540.

Izquierdo, A., Wiedholz, L.M., Millstein, R.A., Yang, R.J., Bussey, T.J., Saksida, L.M., and Holmes, A. (2006). Genetic and dopaminergic modulation of reversal learning in a touchscreen-based operant procedure for mice. Behav. Brain Res. 171, 181-188.

Jayasinghe, V.R., Flores-Barrera, E., West, A.R., and Tseng, K.Y. (2017). Frequency-dependent corticostriatal disinhibition resulting from chronic dopamine depletion: role of local striatal cGMP and GABA-AR signaling. Cereb. Cortex 27, 625-634.

Jin, D.Z., Fujii, N., and Graybiel, A.M. (2009). Neural representation of time in cortico-basal ganglia circuits. Proceedings of the National Academy of Sciences 106, 19156-19161.

Joel, D., Niv, Y., and Ruppin, E. (2002). Actor-critic models of the basal ganglia: new anatomical and computational perspectives. Neural Networks 15, 535-547. 
Kalivas, P.W., Churchill, L., and Klitenick, M.A. (1993). GABA and enkephalin projection from the nucleus accumbens and ventral pallidum to the ventral tegmental area. Neuroscience 57, 1047-1060.

Kawai, T., Yamada, H., Sato, N., Takada, M., and Matsumoto, M. (2015). Roles of the lateral habenula and anterior cingulate cortex in negative outcome monitoring and behavioral adjustment in nonhuman primates. Neuron 88, 792-804.

Kelley, A.E., Smith-Roe, S.L., and Holahan, M.R. (1997). Response-reinforcement learning is dependent on $\mathrm{N}$-methyl-D-aspartate receptor activation in the nucleus accumbens core. Proc. Natl. Acad. Sci. U. S. A. 94, 12174-12179.

Kim, C.K., Ye, L., Jennings, J.H., Pichamoorthy, N., Tang, D.D., Yoo, A.-C.W., Ramakrishnan, C., and Deisseroth, K. (2017). Molecular and circuit-dynamical identification of top-down neural mechanisms for restraint of reward seeking. Cell 170, 1013-1027.e14.

Kim, H., Sul, J.H., Huh, N., Lee, D., and Jung, M.W. (2009). Role of striatum in updating values of chosen actions. J. Neurosci. 29, 14701-14712.

Kim, S., Hwang, J., and Lee, D. (2008). Prefrontal coding of temporally discounted values during intertemporal choice. Neuron 59, 161-172.

Kondo, M., Kobayashi, K., Ohkura, M., Nakai, J., and Matsuzaki, M. (2017). Two-photon calcium imaging of the medial prefrontal cortex and hippocampus without cortical invasion. Elife 6.

Krumin, M., Lee, J.J., Harris, K.D., and Carandini, M. (2018). Decision and navigation in mouse parietal cortex. Elife 7.

Kruzich, P.J., and Grandy, D.K. (2004). Dopamine D2 receptors mediate two-odor discrimination and reversal learning in C57BL/6 mice. BMC Neurosci. 5, 12.

Kwak, S., Huh, N., Seo, J.-S., Lee, J.-E., Han, P.-L., and Jung, M.W. (2014). Role of dopamine D2 receptors in optimizing choice strategy in a dynamic and uncertain environment. Frontiers in Behavioral Neuroscience 8.

Lahiri, A.K., and Bevan, M.D. (2020). Dopaminergic transmission rapidly and persistently enhances excitability of D1 receptor-expressing striatal projection neurons. Neuron 106, 277-290.e6.

Lak, A., Okun, M., Moss, M.M., Gurnani, H., Farrell, K., Wells, M.J., Reddy, C.B., Kepecs, A., Harris, K.D., and Carandini, M. (2020). Dopaminergic and prefrontal basis of learning from sensory confidence and reward value. Neuron 105, 700-711.e6.

Lee, R.S., Mattar, M.G., Parker, N.F., Witten, I.B., and Daw, N.D. (2019). Reward prediction error does not explain movement selectivity in DMS-projecting dopamine neurons. eLife 8.

Long, M.A., Jin, D.Z., and Fee, M.S. (2010). Support for a synaptic chain model of neuronal sequence generation. Nature 468, 394-399.

Lovett-Barron, M., Chen, R., Bradbury, S., Andalman, A.S., Wagle, M., Guo, S., and Deisseroth, K. (2019). Multiple overlapping hypothalamus-brainstem circuits drive rapid threat avoidance. bioRxiv. http://dx.doi.org/10.1101/745075

Luk, C.-H., and Wallis, J.D. (2013). Choice coding in frontal cortex during stimulus-guided or 
action-guided decision-making. J. Neurosci. 33, 1864-1871.

MacAskill, A.F., Cassel, J.M., and Carter, A.G. (2014). Cocaine exposure reorganizes cell type- and input-specific connectivity in the nucleus accumbens. Nature Neuroscience 17, 1198-1207.

Maggi, S., and Humphries, M.D. (2019). Independent population coding of the present and the past in prefrontal cortex during learning. bioRxiv. http://dx.doi.org/10.1101/668962

Maggi, S., Peyrache, A., and Humphries, M.D. (2018). An ensemble code in medial prefrontal cortex links prior events to outcomes during learning. Nat. Commun. 9, 2204.

Matsumoto, M., and Hikosaka, O. (2009). Two types of dopamine neuron distinctly convey positive and negative motivational signals. Nature 459, 837-841.

Matsumoto, N., Minamimoto, T., Graybiel, A.M., and Kimura, M. (2001). Neurons in the Thalamic CM-Pf Complex Supply Striatal Neurons With Information About Behaviorally Significant Sensory Events. Journal of Neurophysiology 85, 960-976.

Montague, P.R., Dayan, P., and Sejnowski, T.J. (1996). A framework for mesencephalic dopamine systems based on predictive Hebbian learning. J. Neurosci. 16, 1936-1947.

Musall, S., Kaufman, M.T., Juavinett, A.L., Gluf, S., and Churchland, A.K. (2019). Single-trial neural dynamics are dominated by richly varied movements. Nat. Neurosci. 22, 1677-1686.

Nauta, W.J., Smith, G.P., Faull, R.L., and Domesick, V.B. (1978). Efferent connections and nigral afferents of the nucleus accumbens septi in the rat. Neuroscience 3, 385-401.

Nestler, E.J. (2001). Molecular basis of long-term plasticity underlying addiction. Nat. Rev. Neurosci. 2, 119-128.

Nicola, S.M., Surmeier, J., and Malenka, R.C. (2000). Dopaminergic modulation of neuronal excitability in the striatum and nucleus accumbens. Annu. Rev. Neurosci. 23, 185-215.

O’Doherty, J.P., Dayan, P., Friston, K., Critchley, H., and Dolan, R.J. (2003). Temporal difference models and reward-related learning in the human brain. Neuron 38, 329-337.

Ölveczky, B.P., Otchy, T.M., Goldberg, J.H., Aronov, D., and Fee, M.S. (2011). Changes in the neural control of a complex motor sequence during learning. J. Neurophysiol. 106, 386-397.

O'Neill, M., and Brown, V.J. (2007). The effect of striatal dopamine depletion and the adenosine A2A antagonist KW-6002 on reversal learning in rats. Neurobiol. Learn. Mem. 88, 75-81.

Otis, J.M., Namboodiri, V.M.K., Matan, A.M., Voets, E.S., Mohorn, E.P., Kosyk, O., McHenry, J.A., Robinson, J.E., Resendez, S.L., Rossi, M.A., et al. (2017). Prefrontal cortex output circuits guide reward seeking through divergent cue encoding. Nature 543, 103-107.

Otis, J.M., Zhu, M., Namboodiri, V.M.K., Cook, C.A., Kosyk, O., Matan, A.M., Ying, R., Hashikawa, Y., Hashikawa, K., Trujillo-Pisanty, I., et al. (2019). Paraventricular thalamus projection neurons integrate cortical and hypothalamic signals for cue-reward processing. Neuron 103, 277-290.e6.

Ottenheimer, D., Richard, J.M., and Janak, P.H. (2018). Ventral pallidum encodes relative reward value earlier and more robustly than nucleus accumbens. Nat. Commun. 9, 4350. 
Ottenheimer, D.J., Wang, K., Haimbaugh, A., Janak, P.H., and Richard, J.M. (2019). Recruitment and disruption of ventral pallidal cue encoding during alcohol seeking. Eur. J. Neurosci. 50, 3428-3444.

Pan, W.-X., Schmidt, R., Wickens, J.R., and Hyland, B.I. (2005). Dopamine cells respond to predicted events during classical conditioning: evidence for eligibility traces in the reward-learning network. J. Neurosci. 25, 6235-6242.

Park, I.M., Meister, M.L.R., Huk, A.C., and Pillow, J.W. (2014). Encoding and decoding in parietal cortex during sensorimotor decision-making. Nature Neuroscience 17, 1395-1403.

Parker, N.F., Cameron, C.M., Taliaferro, J.P., Lee, J., Choi, J.Y., Davidson, T.J., Daw, N.D., and Witten, I.B. (2016). Reward and choice encoding in terminals of midbrain dopamine neurons depends on striatal target. Nat. Neurosci. 19, 845-854.

Parkinson, J.A., Olmstead, M.C., Burns, L.H., Robbins, T.W., and Everitt, B.J. (1999). Dissociation in effects of lesions of the nucleus accumbens core and shell on appetitive pavlovian approach behavior and the potentiation of conditioned reinforcement and locomotor activity by D-amphetamine. J. Neurosci. 19, 2401-2411.

Pastalkova, E., Itskov, V., Amarasingham, A., and Buzsáki, G. (2008). Internally generated cell assembly sequences in the rat hippocampus. Science 321, 1322-1327.

Paxinos, G., and Franklin, K.B.J. (2004). The Mouse Brain in Stereotaxic Coordinates (Gulf Professional Publishing).

Phillips, G.D., Le Noury, J., Wolterink, G., Donselaar-Wolterink, I., Robbins, T.W., and Everitt, B.J. (1993). Cholecystokinin-dopamine interactions within the nucleus accumbens in the control over behaviour by conditioned reinforcement. Behav. Brain Res. 55, 223-231.

Phillips, G.D., Robbins, T.W., and Everitt, B.J. (1994). Mesoaccumbens dopamine-opiate interactions in the control over behaviour by a conditioned reinforcer. Psychopharmacology 114, 345-359.

Phillipson, O.T., and Griffiths, A.C. (1985). The topographic order of inputs to nucleus accumbens in the rat. Neuroscience 16, 275-296.

Picardo, M.A., Merel, J., Katlowitz, K.A., Vallentin, D., Okobi, D.E., Benezra, S.E., Clary, R.C., Pnevmatikakis, E.A., Paninski, L., and Long, M.A. (2016). Population-level representation of a temporal sequence underlying song production in the zebra finch. Neuron 90, 866-876.

Pinto, L., and Dan, Y. (2015). Cell-type-specific activity in prefrontal cortex during goal-directed behavior. Neuron $87,437-450$.

Pnevmatikakis, E.A., and Giovannucci, A. (2017). NoRMCorre: An online algorithm for piecewise rigid motion correction of calcium imaging data. J. Neurosci. Methods 291, 83-94.

Ponzi, A., and Wickens, J. (2010). Sequentially switching cell assemblies in random inhibitory networks of spiking neurons in the striatum. J. Neurosci. 30, 5894-5911.

Poulin, J.-F., Caronia, G., Hofer, C., Cui, Q., Helm, B., Ramakrishnan, C., Chan, C.S., Dombeck, D.A., Deisseroth, K., and Awatramani, R. (2018). Mapping projections of molecularly defined dopamine neuron subtypes using intersectional genetic approaches. Nat. Neurosci. 21, 1260-1271.

Reed, S.J., Lafferty, C.K., Mendoza, J.A., Yang, A.K., Davidson, T.J., Grosenick, L., Deisseroth, K., 
and Britt, J.P. (2018). Coordinated reductions in excitatory input to the nucleus accumbens underlie food consumption. Neuron 99, 1260-1273.e4.

Reynolds, J.N.J., and Wickens, J.R. (2002). Dopamine-dependent plasticity of corticostriatal synapses. Neural Netw. 15, 507-521.

Reynolds, J.N.J., Hyland, B.I., and Wickens, J.R. (2001). A cellular mechanism of reward-related learning. Nature 413, 67-70.

Robbins, T.W., Cador, M., Taylor, J.R., and Everitt, B.J. (1989). Limbic-striatal interactions in reward-related processes. Neuroscience \& Biobehavioral Reviews 13, 155-162.

Roitman, M.F., Wheeler, R.A., and Carelli, R.M. (2005). Nucleus accumbens neurons are innately tuned for rewarding and aversive taste stimuli, encode their predictors, and are linked to motor output. Neuron 45, 587-597.

Russo, S.J., Dietz, D.M., Dumitriu, D., Morrison, J.H., Malenka, R.C., and Nestler, E.J. (2010). The addicted synapse: mechanisms of synaptic and structural plasticity in nucleus accumbens. Trends in Neurosciences 33, 267-276.

Rutledge, R.B., Lazzaro, S.C., Lau, B., Myers, C.E., Gluck, M.A., and Glimcher, P.W. (2009). Dopaminergic drugs modulate learning rates and perseveration in Parkinson's patients in a dynamic foraging task. Journal of Neuroscience 29, 15104-15114.

Sabatini, B.L. (2019). The impact of reporter kinetics on the interpretation of data gathered with fluorescent reporters. bioRxiv. http://dx.doi.org/10.1101/834895

Sakata, J.T., Hampton, C.M., and Brainard, M.S. (2008). Social modulation of sequence and syllable variability in adult birdsong. J. Neurophysiol. 99, 1700-1711.

Salamone, J.D., Steinpreis, R.E., McCullough, L.D., Smith, P., Grebel, D., and Mahan, K. (1991). Haloperidol and nucleus accumbens dopamine depletion suppress lever pressing for food but increase free food consumption in a novel food choice procedure. Psychopharmacology 104 , $515-521$.

Saunders, B.T., Richard, J.M., Margolis, E.B., and Janak, P.H. (2018). Dopamine neurons create Pavlovian conditioned stimuli with circuit-defined motivational properties. Nat. Neurosci. 21, 1072-1083.

Schultz, W. (1998). Predictive reward signal of dopamine neurons. J. Neurophysiol. 80, 1-27.

Schultz, W., Dayan, P., and Montague, P.R. (1997). A neural substrate of prediction and reward. Science 275, 1593-1599.

Setlow, B., Schoenbaum, G., and Gallagher, M. (2003). Neural encoding in ventral striatum during olfactory discrimination learning. Neuron 38, 625-636.

Shen, W., Flajolet, M., Greengard, P., and Surmeier, D.J. (2008). Dichotomous dopaminergic control of striatal synaptic plasticity. Science $321,848-851$.

Siniscalchi, M.J., Wang, H., and Kwan, A.C. (2019). Enhanced population coding for rewarded choices in the medial frontal cortex of the mouse. Cereb. Cortex 29, 4090-4106. 
Steinberg, E.E., Keiflin, R., Boivin, J.R., Witten, I.B., Deisseroth, K., and Janak, P.H. (2013). A causal link between prediction errors, dopamine neurons and learning. Nat. Neurosci. 16, 966-973.

Steinmetz, N.A., Zatka-Haas, P., Carandini, M., and Harris, K.D. (2019). Distributed coding of choice, action and engagement across the mouse brain. Nature 576, 266-273.

Stephenson-Jones, M., Bravo-Rivera, C., Ahrens, S., Furlan, A., Xiao, X., Fernandes-Henriques, C., and Li, B. (2020). Opposing contributions of GABAergic and glutamatergic ventral pallidal neurons to motivational behaviors. Neuron 105, 921-933.e5.

Stuber, G.D., Sparta, D.R., Stamatakis, A.M., van Leeuwen, W.A., Hardjoprajitno, J.E., Cho, S., Tye, K.M., Kempadoo, K.A., Zhang, F., Deisseroth, K., et al. (2011). Excitatory transmission from the amygdala to nucleus accumbens facilitates reward seeking. Nature 475, 377.

Sul, J.H., Kim, H., Huh, N., Lee, D., and Jung, M.W. (2010). Distinct roles of rodent orbitofrontal and medial prefrontal cortex in decision making. Neuron 66, 449-460.

Suri, R.E., and Schultz, W. (1998). Learning of sequential movements by neural network model with dopamine-like reinforcement signal. Experimental Brain Research 121, 350-354.

Suri, R.E., and Schultz, W. (1999). A neural network model with dopamine-like reinforcement signal that learns a spatial delayed response task. Neuroscience 91, 871-890.

Sutton, R.S. (1988). Learning to predict by the methods of temporal differences. Mach. Learn. 3, 9-44.

Sutton, R.S., and Barto, A.G. (1998). Reinforcement Learning: An Introduction (MIT Press).

Swanson, L.W. (1982). The projections of the ventral tegmental area and adjacent regions: a combined fluorescent retrograde tracer and immunofluorescence study in the rat. Brain Res. Bull. 9, 321-353.

Taghzouti, K., Louilot, A., Herman, J.P., Le Moal, M., and Simon, H. (1985). Alternation behavior, spatial discrimination, and reversal disturbances following 6-hydroxydopamine lesions in the nucleus accumbens of the rat. Behav. Neural Biol. 44, 354-363.

Taylor, J., and Robbins, T. (1986). 6-Hydroxydopamine lesions of the nucleus accumbens, but not of the caudate nucleus, attenuate enhanced responding with reward-related stimuli produced by intra-accumbens d-amphetamine. Psychopharmacology 90.

Tecuapetla, F., Carrillo-Reid, L., Bargas, J., and Galarraga, E. (2007). Dopaminergic modulation of short-term synaptic plasticity at striatal inhibitory synapses. Proc. Natl. Acad. Sci. U. S. A. 104, 10258-10263.

Terada, S., Sakurai, Y., Nakahara, H., and Fujisawa, S. (2017). Temporal and Rate Coding for Discrete Event Sequences in the Hippocampus. Neuron 94, 1248-1262.e4.

Tesauro, G. (1992). Practical issues in temporal difference learning. Reinforcement Learning 33-53.

Thévenaz, P., Ruttimann, U.E., and Unser, M. (1998). A pyramid approach to subpixel registration based on intensity. IEEE Trans. Image Process. 7, 27-41.

Tian, J., Huang, R., Cohen, J.Y., Osakada, F., Kobak, D., Machens, C.K., Callaway, E.M., Uchida, N., 
and Watabe-Uchida, M. (2016). Distributed and mixed information in monosynaptic inputs to dopamine neurons. Neuron 91, 1374-1389.

Tindell, A.J., Berridge, K.C., and Aldridge, J.W. (2004). Ventral pallidal representation of pavlovian cues and reward: population and rate codes. J. Neurosci. 24, 1058-1069.

Tsai, H.-C., -C. Tsai, H., Zhang, F., Adamantidis, A., Stuber, G.D., Bonci, A., de Lecea, L., and Deisseroth, K. (2009). Phasic firing in dopaminergic neurons Is sufficient for behavioral conditioning. Science 324, 1080-1084.

Tsitsiklis, J.N., and Van Roy, B. (1997). An analysis of temporal-difference learning with function approximation. IEEE Transactions on Automatic Control 42, 674-690.

Wan, X., and Peoples, L.L. (2006). Firing patterns of accumbal neurons during a pavlovian-conditioned approach task. J. Neurophysiol. 96, 652-660.

Watabe-Uchida, M., Zhu, L., Ogawa, S.K., Vamanrao, A., and Uchida, N. (2012). Whole-brain mapping of direct inputs to midbrain dopamine neurons. Neuron 74, 858-873.

Wei, Wei, W., and Wang, X.-J. (2016). Inhibitory control in the cortico-basal ganglia-thalamocortical loop: complex regulation and interplay with memory and decision processes. Neuron 92, 1093-1105.

Wilson, C.J. (2004). Basal Ganglia. The Synaptic Organization of the Brain 361-414.

Witten, I.B., Steinberg, E.E., Lee, S.Y., Davidson, T.J., Zalocusky, K.A., Brodsky, M., Yizhar, O., Cho, S.L., Gong, S., Ramakrishnan, C., et al. (2011). Recombinase-driver rat lines: tools, techniques, and optogenetic application to dopamine-mediated reinforcement. Neuron 72, 721-733.

Wörgötter, F., and Porr, B. (2005). Temporal sequence learning, prediction, and control: a review of different models and their relation to biological mechanisms. Neural Comput. 17, 245-319.

Wright, C.I., and Groenewegen, H.J. (1995). Patterns of convergence and segregation in the medial nucleus accumbens of the rat: relationships of prefrontal cortical, midline thalamic, and basal amygdaloid afferents. J. Comp. Neurol. 361, 383-403.

Xiong, Q., Znamenskiy, P., and Zador, A. (2015). Selective corticostriatal plasticity during acquisition of an auditory discrimination task. Nature 521, 348-351.

Yagishita, S., Hayashi-Takagi, A., Ellis-Davies, G.C.R., Urakubo, H., Ishii, S., and Kasai, H. (2014). A critical time window for dopamine actions on the structural plasticity of dendritic spines. Science 345 , 1616-1620.

Yang, H., de Jong, J.W., Tak, Y., Peck, J., Bateup, H.S., and Lammel, S. (2018). Nucleus accumbens subnuclei regulate motivated behavior via direct inhibition and disinhibition of VTA dopamine subpopulations. Neuron 97, 434-449.e4.

Zhou, P., Resendez, S.L., Rodriguez-Romaguera, J., Jimenez, J.C., Neufeld, S.Q., Giovannucci, A., Friedrich, J., Pnevmatikakis, E.A., Stuber, G.D., Hen, R., et al. (2018). Efficient and accurate extraction of in vivo calcium signals from microendoscopic video data. Elife 7.

Zhu, Y., Wienecke, C.F.R., Nachtrab, G., and Chen, X. (2016). A thalamic input to the nucleus accumbens mediates opiate dependence. Nature 530, 219-222. 
bioRxiv preprint doi: https://doi.org/10.1101/725382; this version posted July 17,2020 . The copyright holder for this preprint (which was not certified by peer review) is the author/funder. All rights reserved. No reuse allowed without permission.

Zhu, Y., Nachtrab, G., Keyes, P.C., Allen, W.E., Luo, L., and Chen, X. (2018). Dynamic salience processing in paraventricular thalamus gates associative learning. Science 362, 423-429. 